\title{
Commensurability and separability of quasiconvex subgroups
}

\author{
FRÉDÉRIC HAGLUND
}

\begin{abstract}
We show that two uniform lattices of a regular right-angled Fuchsian building are commensurable, provided the chamber is a polygon with at least six edges. We show that in an arbitrary Gromov-hyperbolic regular right-angled building associated to a graph product of finite groups, a uniform lattice is commensurable with the graph product provided all of its quasiconvex subgroups are separable. We obtain a similar result for uniform lattices of the Davis complex of Gromov-hyperbolic two-dimensional Coxeter groups. We also prove that every extension of a uniform lattice of a CAT(0) square complex by a finite group is virtually trivial, provided each quasiconvex subgroup of the lattice is separable.
\end{abstract}

20F55, 20F67, 20F65; 20E26, 51E24, 20E22, 20J06

\section{Introduction}

We consider a special instance of the following (very general) question:

If $X$ and $Y$ are compact metric spaces with isometric universal covers $\tilde{X}$ and $\tilde{Y}$, when do $X$ and $Y$ have isometric compact covers?

Using the fundamental group leads to the following question:

When are two discrete cocompact subgroups of $\operatorname{Is}(\tilde{X})$ commensurable in $\operatorname{Is}(\tilde{X})$ ?

Recall that two subgroups $\Gamma, \Gamma^{\prime}$ of a given group $G$ are said to be commensurable in $G$ when there is $g \in G$ such that $g \Gamma g^{-1} \cap \Gamma^{\prime}$ is of finite index in both $\Gamma^{\prime}$ and $g \Gamma g^{-1}$ (taking $G=\mathbb{R}, \Gamma=\alpha \mathbb{Z}$ and $\Gamma^{\prime}=\alpha^{\prime} \mathbb{Z}$ explains the origin of the word commensurable). Two abstract groups $\Gamma, \Gamma^{\prime}$ are said to be abstractly commensurable if they have finite index isomorphic subgroups.

There is a lot of literature on this subject when $X$ is a locally symmetric space (of noncompact type). In this case $X$ is a Riemannian manifold of nonpositive sectional curvature and $G=\operatorname{Is}(\tilde{X})$ is a Lie group, the set of real points of some algebraic group. It is always possible to construct particular lattices in $G$-called arithmetic because they arise from number theory (see Borel [4]). When the real rank is at least two, 
Margulis showed that all (irreducible) lattices are necessarily arithmetic [32]. Yet all arithmetic lattices need not be commensurable. When the real rank is one, then $X$ is negatively curved. Again there are noncommensurable lattices, because there are nonarithmetic ones by Gromov and Piatetski-Shapiro [17].

We are interested in the case when $\tilde{X}$ is not a manifold anymore, but some particular Tits building or Davis complex, both of which admit a simplicial subdivision. The automorphism groups of these locally compact simplicial complexes are locally compact, totally disconnected, and nondiscrete in many of the cases we are interested in. For us, a lattice of a locally compact simplicial complex $X$ is a subgroup $\Gamma \subset \operatorname{Aut}(X)$ such that each vertex $v$ has a finite stabilizer $\Gamma_{v}$ and $\sum\left(1 /\left|\Gamma_{v}\right|\right)<\infty$, where the sum has one term for each orbit of the $\Gamma$-action on the set of vertices. A lattice $\Gamma \subset \operatorname{Aut}(X)$ is said to be uniform whenever it is cocompact, that is, there are finitely many orbits of vertices.

We first explain our result concerning buildings.

\subsection{Right-angled buildings}

The simplest examples of (nonspherical) buildings are trees. Here any two uniform lattices of a locally finite tree are commensurable (see Bass and Kulkarni [3] and Leighton [31]).

In dimension 2, a Euclidean building $\Delta$ is obtained by taking the product of a $q$-regular tree $T_{q}$ with itself. Burger and Mozes have produced uniform lattices of $\Delta$ which are simple groups [10]. Their construction begins with an irreducible uniform lattice $\Gamma$ in $\Delta$ (of Hilbert modular group nature); by definition, $\Gamma$ is not commensurable with the product of two uniform lattices of the factors. In the same year, Wise constructed non residually finite uniform lattices in $\Delta$, that cannot either be commensurable with a product lattice [40].

Let us describe more precisely $\Delta=T_{q} \times T_{q}$. A face of $\Delta$ is a square, the product of two edges. An edge of $\Delta$ incident to a vertex $v$ is one of two types corresponding to an edge of the first factor or of the second, and there are $q$ of each type. Furthermore any two edges of distinct types coming out of a vertex are contained in one and exactly one square. In other words the link of $\Delta$ at each vertex is the complete bipartite graph on $q+q$ vertices. Note that $\Delta$ is full of copies of the Euclidean plane tesselated by squares. Furthermore $\Delta$ has a nice reducible uniform lattice $\Gamma_{4, q}$ which we describe now.

The free product of two cyclic groups of order $q$ acts on its Bass-Serre tree $T_{q}$ : the action is simply transitive on edges (without inversion). So this free product $L_{q}$ is a uniform lattice of $T_{q}$. We let $\Gamma_{4, q}$ denote the direct product $L_{q} \times L_{q}$.

Algebraic 83 Geometric Topology, Volume 6 (2006) 
The spaces $\Delta$ we want to look at are the hyperbolic analogues of $T_{q} \times T_{q}$, in which squares are replaced by hyperbolic regular right-angled polygons. M Bourdon was the first to consider these right-angled Fuchsian buildings in order to study their geometry à la Gromov. For each integer $q \geq 2$ and each integer $p \geq 4$, there is a connected, simply connected CW-complex $I_{p, q}$ such that

- all attaching maps are injective

- the boundary of a 2-cell is cycle of length $p$

- the link of each vertex is a complete bipartite graph on $q+q$ vertices.

Furthermore such a CW-complex is unique up to cellular homeomorphism [6, Proposition 2.2.1]. Bourdon also introduces a group $\Gamma_{p, q}$ acting on $I_{p, q}$ as a uniform lattice, generalizing the case $p=4$ above. For $p \geq 5$ the group $\Gamma_{p, q}$ is Gromov-hyperbolic, as $I_{p, q}$ can be equipped with a CAT(-1) metric (see Meier [33]).

Our main result answers a question raised by Bourdon [7, Section 1.E.2]. It will follow from a general statement on regular right-angled buildings.

Theorem 1.1 For $p \geq 6$ and $q \geq 2$, all uniform lattices of $I_{p, q}$ are commensurable in $\operatorname{Aut}\left(I_{p, q}\right)$.

Corollary 1.2 For $p \geq 6$ and $q \geq 2$, all uniform lattices of $I_{p, q}$ are $\mathbb{R}$-linear.

Proof of Corollary 1.2 A theorem of Hsu and Wise shows that all $\Gamma_{p, q}$ are linear [27]. Every group abstractly commensurable to a linear group is itself linear (see Wehrfritz [39] for a proof).

Note that B Rémy recently exhibited nonlinear (nonuniform) lattices in $\operatorname{Aut}\left(I_{p, q}\right)$ [35].

The groups $\Gamma_{p, q}$ are special kinds of graph products of finite groups $\left(G_{v}\right)_{v \in V(\mathcal{G})}$ above a finite graph $\mathcal{G}$. To each such graph product $\Gamma=\Gamma\left(\mathcal{G},\left(G_{v}\right)_{v \in V(\mathcal{G})}\right)$ we associate a proper CAT $(0)$ cubical complex $\Delta=\Delta\left(\mathcal{G},\left(G_{v}\right)_{v \in V(\mathcal{G})}\right)$ such that $\Delta$ is a right-angled building and $\Gamma$ acts naturally on $\Delta$ as a uniform lattice (see Section 4 for precise definitions). When $\mathcal{G}$ is a cyclic graph with $p$ vertices and all finite groups $G_{v}$ equal to $\mathbb{Z} / q \mathbb{Z}$, then $\Gamma=\Gamma_{p, q}$ and $\Delta=I_{p, q}$ (more precisely $\Delta$ is the first square subdivision of the polygonal complex $I_{p, q}$ ).

We will prove the following criterion:

Theorem 1.3 Suppose $\Delta\left(\mathcal{G},\left(G_{v}\right)_{v \in V(\mathcal{G})}\right)$ is Gromov-hyperbolic. Then a uniform lattice $\Gamma^{\prime}$ is commensurable to $\Gamma$ in $\operatorname{Aut}(\Delta)$ if all of its quasiconvex subgroups are separable. 
Here $\operatorname{Aut}(\Delta)$ is the group of type-preserving automorphisms (see Section 4). The hyperbolicity condition is equivalent to the fact that the graph $\mathcal{G}$ does not contain a square as a full subgraph (see Gromov [16]).

Recall that a subgroup $\Lambda$ of a Gromov-hyperbolic group is quasiconvex if a $\Gamma$-geodesic between two elements of $\Lambda$ stays at uniformly bounded distance of $\Lambda$. Recall also that a subgroup $\Lambda$ of a group $\Gamma$ is separable if it is the intersection of all finite index subgroups containing it. The residual finiteness of $\Gamma$ is equivalent to the separability of the trivial subgroup.

The property that all quasiconvex subgroups be separable has been studied for Fuchsian and Kleinian groups by Scott [38], Gitik [14], and Agol, Long and Reid [1]. In [41] and [43], Wise was the first to explore this property in the framework of geometric group theory. He also showed the importance of this property with respect to the problem of residual finiteness of Gromov-hyperbolic groups (see Kapovich and Wise [30]).

We proved in [22] the converse of Theorem 1.3 by establishing the separability of all quasiconvex subgroups of graph products of finite groups. Hence in the world of Gromov-hyperbolic right-angled buildings the commensurability class of the "natural" uniform lattice is characterized by the separability of quasiconvex subgroups.

Theorem 1.1 is a consequence of Theorem 1.3 since $I_{p, q}$ is hyperbolic for $p \geq 5$. A remarkable theorem of Wise [41, Theorem 10.1] insures that for $p \geq 6$ and $q \geq 2$, all quasiconvex subgroups of any uniform lattice of $I_{p, q}$ are separable. Note that the group of type-preserving automorphisms of $I_{p, q}$ is of finite index in the full automorphism group. So Theorem 1.1 is valid even if we understand $\operatorname{Aut}\left(I_{p, q}\right)$ as the full automorphism group. Observe that in general the group of type-preserving automorphisms of a right-angled building is not of finite index in the full automorphism group.

In the case $p=5$ the criterion of Theorem 1.3 remains valid, but Wise's separability result is not available. So the question remains open.

To establish Theorem 1.3 we first introduce a geometric invariant of the action of the uniform lattice $\Gamma^{\prime}$ called holonomy which is trivial for a naturally defined finite index subgroup of $\Gamma^{\prime}=\Gamma$. In Section 6, we prove the following:

Theorem 1.4 A uniform lattice $\Gamma^{\prime}$ is commensurable to $\Gamma$ in $\operatorname{Aut}(\Delta)$ if and only if $\Gamma^{\prime}$ has a finite index subgroup with trivial holonomy.

In Section 7, we conclude the proof of Theorem 1.3 by progressively killing all the holonomy in a convenient finite index subgroup of $\Gamma^{\prime}$. This is where the separability is used. 
Note that Theorem 1.4 can be used to recover a previous result by Januszkiewicz and Swiatkowski [28] (see Section 6).

Corollary 1.5 The graph products of $\Gamma\left(\mathcal{G},\left(G_{v}\right)_{v \in V(G)}\right)$ and $\Gamma\left(\mathcal{G},\left(H_{v}\right)_{v \in V(G)}\right)$ are commensurable if for each vertex $v$, the two finite groups $G_{v}$ and $H_{v}$ have the same cardinality.

Locally finite biregular trees are the simplest infinite regular right-angled buildings we can consider. But in the case of a tree the holonomy is automatically trivial for any uniform lattice. Hence in Section 6 we also recover "Leighton's lemma" $[2 ; 31 ; 3]$.

Corollary 1.6 Two uniform lattices of a locally finite biregular tree $T$ are commensurable in $\operatorname{Aut}(T)$.

\subsection{Davis complexes}

We now turn to our second result linking commensurability and separability of quasiconvex subgroups. For some Fuchsian buildings which are not right-angled we can still prove the commensurability of all uniform lattices. By Davis [11] and Haglund [19], such a Fuchsian building is Davis' geometric realization of a Coxeter system whose finite nerve $L$ is a generalized $\mu$-gon with $\mu \geq 3$, and all finite entries of the Coxeter matrix are equal to some integer $m \geq 2$. In fact our result is obtained in the framework of the Davis complexes, the basic properties of which we recall now.

Let $(m, L)$ be a pair where $m$ is any integer and $L$ is any graph such that $m \geq 3$ or $m=2$ and the girth of $L$ is $\geq 4$. The Davis complex associated to $(m, L)$ is a CAT(0) polygonal complex $X(m, L)$ in which each polygon has $2 m$ edges and the link of each vertex is isomorphic to $L$. The automorphism group of $X(m, L)$ contains a subgroup $W(m, L)$ generated by reflections of $X(m, L)$ which acts simply transitively on vertices. In this context we define a reflection to be an order-2 automorphism exchanging the endpoints of some edge $e$ of $X(m, L)$, and fixing pointwise the totally geodesic tree consisting of points equidistant to the endpoints of $e$. Note that the (locally compact) automorphism group of the polygonal complex $X(m, L)$ is not discrete if and only if $L$ has nontrivial automorphisms fixing pointwise the star of a vertex $[19 ; 23]$.

When $L$ is a generalized $\mu$-gon, $X(m, L)$ is Davis' geometric realization of a building whose apartments are tesselations of $\mathbb{M}^{2}$ by regular $2 m$-gons with vertex angle $\pi / \mu$. (In the terminology of [20] these buildings are locally reflexive without holonomy). For $\mu=2$ and $L$ finite, we recover regular locally finite right-angled buildings for which Theorem 1.3 implies uniform lattices are commensurable.

In Section 14 we will prove the following generalization. 
Theorem 1.7 Assume $L$ is a finite graph and $X(m, L)$ is negatively curved (that is $m \geq 4$, or $m \geq 3$ and the girth of $L$ is at least 4 , or $m \geq 2$ and the girth of $L$ is at least $5)$.

Then a uniform lattice $\Gamma$ in $X(m, L)$ is commensurable to the Coxeter group $W(m, L)$ in $\operatorname{Aut}(X(m, L))$ if all of its quasiconvex subgroup are separable.

Corollary 1.8 If $L$ is a finite bipartite graph and $m \geq 3$ then all uniform lattices of $X(m, L)$ are commensurable (hence they are linear). In particular all uniform lattices of a given locally reflexive even-gonal Fuchsian buildings without holonomy are commensurable.

Proof The bipartite assumption implies that the first square subdivision of $X(m, L)$ is a VH-complex of Wise (this argument is implicit in the proof of Theorem 10.1 of [41]). By Theorem 8.1 of [41], all uniform lattices of $X(m, L)$ have separable quasiconvex subgroups. Thus the corollary is a direct application of Theorem 1.7.

The converse of Theorem 1.7 is true at least when $m=2$ [22] or more generally when $m$ is even (the method we developed in [22] for graphs of finite groups also applies to this particular kind of Coxeter groups). So once again in these complexes $X(m, L)$ there is a preferred commensurability class of uniform lattices, characterized by important separability properties.

The proof of Theorem 1.7 is parallel to the proof of Theorem 1.3, although we were not able to find a common proof for these results. Here we use the notion of systems of local reflections and of their holonomy which we introduced in [18] and [20]. The basic system of local reflections is given by the restrictions of the reflections of $W(m, L)$ to the neighbourhoods of edges. Its holonomy is obtained by composing these reflections along the boundary of a polygon: this is trivial by definition of the relations of a Coxeter group. In Section 14 we obtain an analogy to Theorem 1.4.

Theorem 1.9 A uniform lattice $\Gamma$ of $X=X(m, L)$ is commensurable to $W(m, L)$ in $\operatorname{Aut}(X)$ if and only if $\Gamma$ has a finite index subgroup preserving a system of local reflections whose holonomy is trivial.

In order to deduce Theorem 1.7 from Theorem 1.9 it remains to use the separability of certain quasiconvex subgroups to produce a system of local reflections without holonomy preserved by a finite index subgroup of the uniform lattice $\Gamma$.

Here is a sketch of the argument. Passing to a finite index subgroup, we may assume that $\Gamma$ preserves a system $\sigma$ of local reflections. An easy computation shows that 
the holonomy of $\sigma$ splits into two parts. We then consider a kind of walls inside the polygonal complex. These walls are always quasiconvex under the negative curvature assumption, and so are their stabilizers (as well as the finite index subgroups of these). This allows us to modify $\sigma$ along the $\Gamma$-orbit of one wall $M$ in such a way that the new system of local reflections $\sigma^{\prime}$ is invariant under a finite index subgroup of $\Gamma$. Furthermore the holonomy of $\sigma^{\prime}$ at polygons transverse to a $\Gamma$-translate of $M$ is "half-trivial". There are finitely many orbits of walls, so if we iterate this process a finite number of times we get a finite index subgroup of $\Gamma$ preserving a system of local reflections without holonomy.

\subsection{Extensions by finite groups}

In the two situations above we noticed that quasiconvex subgroup separability could be used to make certain conjugation obstructions virtually vanish. We apply this idea once more to answer a question raised by Anna Erschler:

If $\Gamma$ is a group studied by Wise in [41] and $1 \rightarrow F \rightarrow G \rightarrow \Gamma \rightarrow 1$ is an exact sequence of groups with $F$ finite, is $G$ always residually finite? commensurable with $\Gamma$ ?

This is a natural question since Ragunathan proved in [34] that a uniform lattice of Spin $(2, n), n$ odd, always admits a finite extension which is not virtually torsion-free, hence not commensurable with the initial lattice.

The groups studied by Wise in [41] are fundamental groups of certain nonpositively curved square complexes. In the universal covers of such spaces there is a natural notion of wall: an equivalence class for the relation on edges generated by being opposite inside one square. The stabilizer of a wall is quasiconvex. In more general polygonal complexes there are several natural notions of walls. We choose one and introduce a combinatorial condition $\left(\mathrm{C}^{2}\right)$ on a polygonal complex $X$ so that when $\left(\mathrm{C}^{2}\right)$ is fulfilled our walls are automatically convex for a piecewise Euclidean nonpositively curved metric on $X$. We say that a subgroup of the fundamental group of a $\left(\mathrm{C}^{2}\right)$ polygonal complex $X$ is convex whenever it preserves a convex subset $Y \subset \widetilde{X}$ of the universal cover and acts cocompactly on $Y$.

Theorem 1.10 Let $X$ denote a compact polygonal complex satisfying the nonpositive curvature condition $\left(\mathrm{C}^{2}\right)$, for example, $X$ is a nonpositively curved square complex. Assume that convex subgroups are separable in $\Gamma=\pi_{1}(X)$.

Then for any extension $1 \rightarrow G \rightarrow \bar{\Gamma} \rightarrow \Gamma \rightarrow 1$ where $G$ is finite, the group $\bar{\Gamma}$ is commensurable with $\Gamma$. 
Any square complex studied in [41] satisfies the above assumptions and its fundamental group is residually finite (in fact every quasiconvex subgroup is separable). In particular any extension of such a fundamental group is also residually finite.

\section{Outline of paper}

The paper is organized in three parts. Theses part are independent, except that we will use in the last part some (classical) definitions introduced in the previous parts.

In the first part we deal with (regular) right-angled buildings. We identify buildings with their Davis-Moussong realization. In Section 3 we recall basic definitions and facts on cube complexes. This is mostly dedicated to the reader more familiar with chamber systems and "abstract" buildings than with CAT(0) cube complexes.

In Section 4 we define the right-angled building of a graph product as a (typed) CAT(0) cube complex, on which the graph product acts as a uniform lattice. We also introduce residues, a kind of typed subcomplex. We are particularly interested in a specific kind of residues that will play the role of "walls". We establish a product structure for these wall-residues, where the first factor is compact. All the results in this section are rather classical, especially for the reader used to the langauge of chamber systems.

In Section 5 we define the holonomy of a uniform lattice at a wall-residue to be the projection of the residue stabilizer on the automorphism group of the compact factor. Thus it is a finite subgroup. We note that a naturally defined finite index subgroup of the graph product has trivial holonomy.

We define atlases on our building $\Delta$ in Section 6. We prove that the automorphism group of an atlas is discrete cocompact, and that any two atlases are conjugate in $\operatorname{Aut}_{0}(\Delta)$. We also note that the graph product preserves a naturally defined atlas. So we have a strategy for commensurating a uniform lattice $\Gamma$ with the graph product: find an atlas on the building which is invariant under some finite index subgroup of $\Gamma$. Since in fact groups without holonomy always preserve an atlas, it remains to find a finite index subgroup without holonomy. We do this in Section 7 using the separability of the wall-residues stabilizers and their finite index subgroups.

In the second part we study extensions by finite groups. We first introduce many basic definitions on polygonal complexes (Section 8), which will be used also in the third part.

Then we define the walls of a polygonal complex (Section 9). When the number of sides of each polygon is even, our walls are essentially obtained as unions of segments joining inside a polygon the midpoint of an edge to the midpoint of the opposite edge. We

Algebraic 83 Geometric Topology, Volume 6 (2006) 
check that these walls correspond to totally geodesic subtrees when some nonpositive curvature condition is fulfilled.

In Section 10 we consider a 2-cocycle (with values in a finite abelian group) on a compact polygonal complex satisfying our particular nonpositive curvature condition. We show that if the fundamental group has separable quasiconvex subgroups then there is a finite cover on which the lifted 2-cocycle vanishes. This proves Theorem 1.10.

In the last part we study commensurability with Coxeter groups. We use definitions given in the second part and adapt the argument there to the noncommutative context.

In Section 11 we recall the definition and the properties of the Davis-Moussong realization of a Coxeter group. We will work with two-dimensional Coxeter groups, so our complexes are polygonal, and the Coxeter group acts on it as a uniform lattice. We introduce a new type of walls in polygonal complexes, which we call $e$-walls, in Section 12.

In Section 13 we define systems of local reflections and their holonomy. Here, a system of local reflection without holonomy plays the same role as an atlas on a regular rightangled building. We note that any two systems of local reflections without holonomy are conjugate in the full automorphism group, and the automorphism group of any such system is a finite extension of a conjugate of the initial Coxeter group. So in order to commensurate a uniform lattice $\Gamma$ with our initial Coxeter group, it is enough to produce a system of local reflections without holonomy which is preserved by a finite index subgroup of $\Gamma$. This is done in Section 14, using a nonpositive curvature assumption and the additional hypothesis that $e$-walls stabilizers in $\Gamma$ and their finite index subgroups are separable.

In the three situations we deal with, we note that the obstructions for commensurability lie along wall-like convex subcomplexes. Under strong enough separability assumptions it is then possible to kill these obstructions in a finite index subgroup, thus getting the commensurability result.

The results in this paper raise some questions.

Problem 2.1 Let $1 \rightarrow G \rightarrow \bar{\Gamma} \rightarrow \Gamma \rightarrow 1$ denote a central extension of $\Gamma$, a uniform lattice of a (Gromov-hyperbolic) CAT(0) cube complex of dimension $n$ at least 3 . Does the separability of quasiconvex subgroups of $\Gamma$ imply the virtual triviality of the extension?

Note that our answer is for $n=2$. In higher dimensions, our method does not apply without changes. Indeed we are not able to kill naturally a 2-cocycle along the walls, even on a single 3 -cube.

Algebraic $8 \mathcal{G}$ Geometric Topology, Volume 6 (2006) 
The same question arises for commensurability of uniform lattices with Coxeter groups inside Davis complexes of higher dimension:

Problem 2.2 Let $(W, S)$ denote some right-angled Coxeter system, that is, a graph product of order two groups along some graph $\mathcal{G}$. Assume that the Davis complex $X$ of $(W, S)$ has dimension $n \geq 3$, so that $\mathcal{G}$ contains a complete graph on 4 vertices. Does the separability of quasiconvex subgroups of a uniform lattice $\Gamma$ imply that $\Gamma$ is commensurable with $W$ ?

Problem 2.3 Find a Gromov-hyperbolic CAT(0) square complex that admits two noncommensurable uniform lattices.

Of course it would be even more interesting if the square complex was the DavisMoussong realization of a right-angled Coxeter group. Note that the product of two (regular nonelementary) trees admits noncommensurable uniform lattices. That is why we insist here on Gromov-hyperbolicity.

With Wise [26], we introduced a notion of special uniform lattice $\Gamma$ of a CAT(0) cube complex $X$ : we say that $\Gamma$ is special if there exists an injective morphism from $\Gamma$ into a finitely generated right-angled Coxeter group $W$, and an equivariant isomorphism of $X$ onto a convex subcomplex of the Davis realization of $W$.

Problem 2.4 Are two special uniform lattices of a locally compact CAT( 0 ) cube complex always commensurable?

For example, a uniform lattice of a product of two trees is (virtually) special exactly when it is virtually a product of uniform lattices of the trees. So in this case all special uniform lattices are commensurable.

\section{Part I Commensurability of uniform lattices in right-angled buildings}

\section{Cube complexes}

In this section we recall basic definitions and facts about cube complexes (see also [8, page 111]).

Algebraic 83 Geometric Topology, Volume 6 (2006) 
Definition 3.1 A cube complex of dimension 0 is a discrete, nonempty set. Any map $f: X \rightarrow Y$ between cube complexes of dimension 0 is said to be combinatorial. If $C$ is a singleton and $X$ is a cube complex of dimension 0 , then any map $f: C \rightarrow X$ is called a $0-$ cube of $X$. Observe that the boundary of any unit interval is a cube complex of dimension 0 .

Assume that cube complexes of dimension $m \leq n$, combinatorial maps between such spaces, and also $k$-cubes of such a space for $k \leq m$ have been defined. Then a cube complex of dimension $n+1$ is obtained by gluing $(n+1)$-cubes to some cube complex of dimension $n$. More precisely, let $X^{n}$ denote some cube complex of dimension $n$, and let $\left(f_{i}\right)_{i \in I}$ denote some (nonempty) family of combinatorial maps $f_{i}: \partial C_{i} \rightarrow X^{n}$, where $C_{i}$ is some Euclidean cube of dimension $n+1$ whose edges have length 1 and $\partial C_{i}$ denotes the combinatorial boundary of $C_{i}$ endowed with its natural cube complex structure of dimension $n$. In fact we assume by induction that a cube complex structure of dimension $n$ is defined on the union $C^{n}$ of faces of dimension $n$ of any unit Euclidean cube $C$ of dimension $p \geq n$, in such a way that for any $p$-face $D$ of a unit Euclidean cube $C$ with $p \geq n$, the natural inclusion $D^{n} \rightarrow C^{n}$ is combinatorial.

We obtain a cube complex $X$ of dimension $n+1$ if we glue each cube $C_{i}$ to $X^{n}$ along $\partial C_{i}$ using the maps $f_{i}$. Then $X^{n}$ is naturally a subspace of $X$, called its $n$-skeleton. Observe that the maps $f_{i}: \partial C_{i} \rightarrow X^{n}$ naturally extend to maps $f_{i}: C_{i} \rightarrow X$. These maps are the $(n+1)$-cubes of $X$ for $k \leq n$ the $k$-cubes of $X$ are the $k$-cubes of $X^{n}$. Now we define a map $f: X \rightarrow Y$ between cube complexes of dimension $n+1$ to be combinatorial whenever it maps $X^{n}$ into $Y^{n}$, the induced map between the $n$-skeleta is combinatorial and for each $(n+1)$-cube $f_{i}: C_{i} \rightarrow X$ there exists an $(n+1)$-cube $g_{j}: D_{j} \rightarrow Y$ and an isometry $h: C_{i} \rightarrow D_{j}$ such that $f f_{i}=g_{j} h$.

For any topological space $X^{\prime}$, a cube complex structure of dimension $n+1$ is given by a homeomorphism $h: X \rightarrow X^{\prime}$, where $X$ is a cube complex constructed as above. Two homeomorphisms $h_{1}, h_{2}: X \rightarrow X_{1}^{\prime}, X_{2}^{\prime}$ define the same structure whenever $h_{2} \circ h_{1}{ }^{-1}$ is combinatorial. Pushing by $h$ defines the $n$-skeleton of $X^{\prime}$ and the $(n+1)$-cubes of $X^{\prime}$. Combinatorial maps between topological spaces equipped with a cube complex structure of dimension $n+1$ are similarly defined. The open cubes of $X^{\prime}$ are the image of the interior of $C$ by some cube $C \rightarrow X^{\prime}$. Clearly $X^{\prime}$ is the disjoint union of its open cubes: every point in $X^{\prime}$ is contained in the interior of a unique cube.

It remains to define a natural cube complex structure of dimension $n+1$ on the union $C^{n+1}$ of faces of dimension $n+1$ of any unit Euclidean cube $C$ of dimension at least $n+1$. Let $C^{n}$ denote the union of the $n$-faces of $C$. By induction this is a cube complex of dimension $n$. For any $(n+1)$-face $F$ of $C$, we have $\partial F=F^{n}$ and we know by induction that the inclusion $F \rightarrow C$ induces a combinatorial map $F^{n} \rightarrow C^{n}$. 
The cube complex obtained by gluing all $(n+1)$-faces to the $n$-skeleton $C^{n}$ has a natural homeomorphism to $C^{n+1}$. The naturality of this structure is straightforward.

We give the usual names to low dimensional cubes: 0 -cubes are vertices, 1-cubes are edges, 2-cubes are squares. We sometimes identify $k$-cubes with their range (note that distinct cubes have distinct ranges). Thus the 0 -skeleton may be viewed as the set of vertices.

A subcomplex of a cube complex is a union of cubes. It inherits naturally a cube complex structure for which the inclusion is combinatorial.

Definition 3.2 A multisimplicial complex $X$, a $k$-simplex of $X$, a combinatorial map $X \rightarrow Y$ between multisimplicial complexes and a subcomplex are obtained from Definition 3.1 by replacing unit Euclidean cubes by affine simplices (isometries of cubes have to be replaced by affine isomorphism of simplices). Observe that a combinatorial map preserves the dimension of simplices.

A simplicial complex is a multisimplicial complex $X$ such that each simplex is injective and two distinct $k$-simplices $f, f^{\prime}: \Sigma^{k} \rightarrow X$ have distinct boundaries, ie, $f\left(\partial \Sigma^{k}\right) \neq f^{\prime}\left(\partial \Sigma^{k}\right)$. It is easy to check that these simplicial complexes are in one-toone correspondence with abstract simplicial complexes, ie, collections of nonempty subsets, called simplices, of a given set $I$ such that if $\sigma$ is a simplex and if $\tau$ is a nonempty subset of $\sigma$, then $\tau$ is a simplex too. For any simplicial complex $N$ we will denote by $\bar{N}$ the poset ordered by inclusion whose elements are the simplices of $N$, together with the empty set.

The join of two (abstract) simplicial complexes $X, Y$ is the simplicial complex $X * Y$ with vertex set $X^{0} \sqcup Y^{0}$ and with simplices the subsets $\sigma \cup \tau$, for $\sigma \in \bar{X}, \tau \in \bar{Y}$. If $\sigma, \tau$ are two simplices of a simplicial complex $X$ such that $\sigma \cup \tau$ is a simplex, we say that $\sigma$ and $\tau$ are joinable in $X$. The join is commutative and associative.

A simplicial complex $L$ is said to be a flag if any complete subgraph of the 1-skeleton is the 1-skeleton of a unique simplex of $L$ (a complete subgraph of $L^{1}$ is a subcomplex $K$ such that any two vertices of $K$ are contained in an edge of $K$ ).

For a reference on simplicial complexes see Rourke and Sanderson [37, Chapter 2].

Example 3.3 (Thickened octahedron) A thickened octahedron is a simplicial complex $L$ obtained by iterated joins with discrete sets.

More precisely let $L_{1}, \cdots, L_{n}$ denote simplicial complexes of dimension 0 . Then $L=L_{1} * \cdots * L_{n}$ is a thickened octahedron. 
Assume that a group $G$ acts simplicially on a simplicial complex $L$ and that there is a simplex $\sigma=\left\{x_{0}, x_{1}, \ldots, x_{n}\right\}$ of $L$ such that $\sigma$ is a strict fundamental domain for the action of $G$ on $L$, ie, the orbit of any simplex of $L$ under $G$ contains exactly one simplex of $\sigma$. Let $G_{i}$ denote the stabilizer of $\sigma_{i}=\left\{x_{0}, x_{1}, \ldots, \widehat{x}_{i}, \ldots, x_{n}\right\}$. Then $L$ is a thickened octahedron provided $G$ is generated by $\left\{G_{i}\right\}_{i \in I}$ and $\left[G_{i}, G_{j}\right]=1$ for $i \neq j$.

To check this, let $L\left(x_{0}\right)$ denote the subcomplex of $L$ consisting in those simplices not containing $x_{0}$ but joinable with $\left\{x_{0}\right\}$. Observe that the stabilizer $G\left(x_{0}\right)$ of $x_{0}$ in $G$ acts on $L\left(x_{0}\right)$ with $\sigma_{0}$ as a strict fundamental domain. The stabilizers of the codimension-one faces of $\sigma_{0}$ are precisely $G_{1}, \ldots, G_{n}$, which commute by assumption. We claim that $G\left(x_{0}\right)$ is generated by $G_{1}, \ldots, G_{n}$. Indeed for any $g \in G$ we have $g=g_{0} h=h g_{0}$ where $g_{0} \in\left\langle G_{1}, \ldots, G_{n}\right\rangle$ and $h \in G_{0}$. It follows that $g(\sigma)=g\left(x_{0} *\right.$ $\left.\sigma_{0}\right)=k\left(x_{0}\right) * g_{0}\left(\sigma_{0}\right)$. Thus if $g \in G\left(x_{0}\right)$ then $k\left(x_{0}\right)=x_{0}$, whence $k \in \cap_{i=0}^{i=n} G_{i}$ and $g \in\left\langle G_{1}, \ldots, G_{n}\right\rangle$. Since $\sigma$ is a fundamental domain, the formula $g(\sigma)=k\left(x_{0}\right) * g_{0}\left(\sigma_{0}\right)$ also shows that $L$ is the join of $L\left(x_{0}\right)$ and the set of vertices joinable with $\sigma_{0}$. We conclude that $L$ is a thickened octahedron by induction on the dimension of $\sigma$.

Definition 3.4 (Links) Let $C$ be a Euclidean cube and let $p$ be one of its vertices. We define $\operatorname{link}(p, C)$ as the (codimension 1) simplex generated by the midpoints of edges of $C$ containing $p$. Note that if $D$ is a strict face of $C$ containing $p$ then $\operatorname{link}(p, D) \subset \partial \operatorname{link}(p, C)$.

Let $X$ be a cube complex and let $v$ be a vertex of $X$. For each based $(k+1)-$ cube $f:(C, p) \rightarrow(X, v)$ we obtain by restriction a map $\sigma_{f}: \operatorname{link}(p, C) \rightarrow X$. The link of $X$ at $v$ is the multisimplicial complex $\operatorname{link}(v, X) \subset X$ such that each $k$-simplex is $\sigma_{f}$ for some $f$. We say that $X$ is locally finite if for each vertex $v$, the complex $\operatorname{link}(v, X)$ has finitely many vertices.

We define similarly the link of a vertex in a multisimplicial complex. The link of a vertex in a simplicial complex is still simplicial.

We say that $X$ is a simple cube complex if for each vertex $v$ the complex $\operatorname{link}(v, X)$ is a simplicial complex. This amounts to demanding that the cubes of $X$ are locally injective, and any combinatorial map to $X$ defined on the union of faces containing a given vertex of a cube extends to at most one combinatorial map on the whole cube.

The following construction appears in Davis, Januszkiewicz and Scott [13, 1.2 page 503].

Lemma 3.5 Let $N$ denote any simplicial complex. Then there exists a cubical complex $C(N)$ with a distinguished vertex $v$, called the center of $C(N)$, such that 
$\operatorname{link}(v, C(N))$ is isomorphic to $N$, and for every cube $C$ of $C(N)$ there exists a unique minimal cube $Q(C)$ containing $v \cup C$. Moreover such a cubical complex is unique up to combinatorial isomorphism preserving the centers. The complex $C(N)$ is called the cubical cone on $N$. For every combinatorial map $f: N \rightarrow M$ there is a unique combinatorial map $c(f): C(N) \rightarrow C(M)$ inducing $f$ on the link of the center of $C(N)$. We will denote by $\partial C(N)$ the union of cubes not containing $v$.

For any cube $C$ of $C(N), C \neq\{v\}$, the cube $Q(C)$ defines a certain simplex $t(C)$ in $\operatorname{link}(v, C(N))=N$. Thus we get a map $t$ assigning to each vertex $x$ of $C(N), x \neq v$, the simplex $t(\{x\})$ of $N$. We extend the definition of $t$ by declaring $t(v)=\varnothing$. On the set of vertices of each cube of $C(N)$ the map $t$ is a bijection onto some interval of the poset $\bar{N}$.

The greatest element of this interval is $t(C)$. We will denote by $\underline{t}(C)$ the least element of this interval. For each $p \in C(N)$ let $C(p)$ denote the unique cube of $C(N)$ containing $p$ in its interior. Then we define $t(p)$ and $\underline{t}(p)$ as $t(C(p))$ and $\underline{t}(C(p))$. The map $t$ is the typing map on $C(N)$, and $t(p)$ is the type of the point $p$.

The map $t$ induces a bijection from the set of vertices of the cubical cone $C(N)$ onto $\bar{N}$.
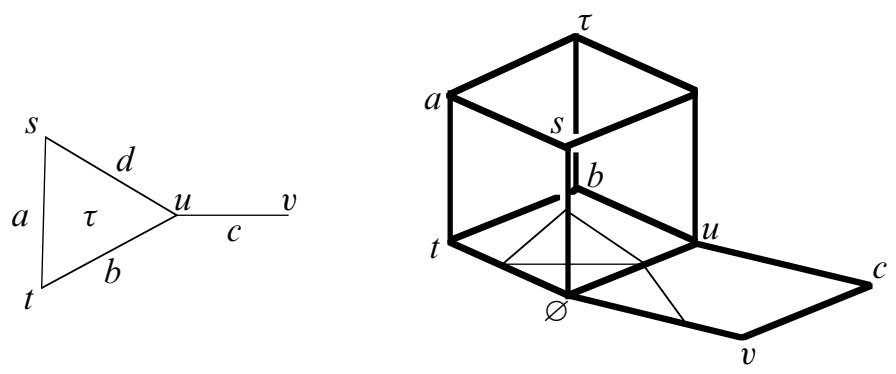

Figure 1: $N$ with the cubical cone on $N$ and typing map. On the left, $N$ has four vertices $s, t, u, v$, four edges $a, b, c, d$ and one triangle $\tau$.

Proof: Uniqueness Let $(C, v)$ be a cube complex as in the lemma, and let $\left(C^{\prime}, v^{\prime}\right)$ denote any other based cube complex endowed with an isomorphism $\varphi$ from $\operatorname{link}(v, C)$ to $\operatorname{link}\left(v^{\prime}, C^{\prime}\right)$. For any cube $Q$ of $C$ containing $v$ there is a (unique) combinatorial map $f_{Q}: Q \rightarrow C^{\prime}$ inducing $\varphi$ on $\operatorname{link}(v, Q)$. For any cube $\xi$ of $C$ we define $f_{\xi}$ as the restriction of $f_{Q(\xi)}$ to $\xi$. These maps fit together and produce a based combinatorial map $f:(C, v) \rightarrow\left(C^{\prime}, v^{\prime}\right)$ inducing $\varphi$ on $\operatorname{link}(v, C)$. The uniqueness of such a map is straightforward. The universal property we have just described implies as usual the uniqueness of the based cube complex $(C, v)$ up to isomorphism. 
Existence In the Hilbert space $\ell^{2}\left(N^{0}\right)$ consider the union of cubes whose vertices are vectors of the form: $\sum_{v \in \sigma} x_{v} e_{v}$, where $\left(e_{v}\right)_{v \in N^{0}}$ denotes the canonical Hilbert basis, $x_{v}=0$ or 1 , and $\sigma$ denotes some simplex of $N$.

If $f: N \rightarrow M$ is a combinatorial map, it induces a linear map $\vec{f}: \ell^{2}\left(N^{0}\right) \rightarrow \ell^{2}\left(M^{0}\right)$. Restricting this to $C(N)$ yields a combinatorial map $c(f): C(N) \rightarrow C(M)$ with the desired property. Two combinatorial maps $C(N) \rightarrow C(M)$ which induce $f$ on $\operatorname{link}(v, C(N))$ have to agree on each cube containing $v$. Uniqueness then follows because each cube of $C(N)$ is contained in a cube containing $v$.

Properties of the map $t$ Let $Q$ be a cube containing $v$. A vertex $x$ of $C$ is opposite to $v$ in $Q$ if and only if $Q(\{x\})=Q$. So for the vertex $v_{Q}$ opposite to $v$ in $Q$ we have $t\left(\left\{v_{Q}\right\}\right)=\operatorname{link}(v, Q)$. For any cube $C$ of $C(N)$ we set $v_{C}=v_{Q(C)}$. Note that $v_{C}$ is a vertex of $C$. Finally define $w_{C}$ to be the vertex of $C$ opposite to $v_{C}$ in $C$. Observe that the cube $Q(C)$ is the product of $C$ and a unique face $q(C)$ containing $v$ and meeting $C$ at the single vertex $w_{C}$. For example, $w_{C}=v$ if and only if $v \in C$. Then for any vertex $x$ of $C$, the smallest cube containing $\{v\} \cup\{x\}$ in fact contains $w_{C}$ and is contained in $Q(C)=Q\left(\left\{v_{C}\right\}\right)$. Thus we get $t(q(C)) \subset t(\{x\}) \subset t(Q(C))$. Clearly $t$ is injective on the set of 0 -cubes. So $t$ induces a bijection between the set of vertices of $C$ and the interval $\{\tau \in \bar{N}, t(q(C)) \subset \tau \subset t(Q(C))\}$. Since $t: C^{0} \rightarrow \bar{N}$ is clearly onto it is in fact a bijection.

Lemma 3.6 The cubical cone of a join is the product of the cubical cones.

More precisely let $N_{1}, N_{2}$ be two abstract simplicial complexes, with vertex sets $I_{1}, I_{2}$. Let $N=N_{1} * N_{2}$ denote the join of $N_{1}$ and $N_{2}$.

Then there is a combinatorial isomorphism $j: C\left(N_{1}\right) \times C\left(N_{2}\right) \rightarrow C(N)$ such that $t\left(j\left(p_{1}, p_{2}\right)\right)=t_{1}\left(p_{1}\right) * t_{2}\left(p_{2}\right) \subset I=I_{1} \sqcup I_{2}$. Such an isomorphism is unique.

Proof For existence, consider the natural isometry $\ell^{2}\left(I_{1}\right) \times \ell^{2}\left(I_{2}\right) \rightarrow \ell^{2}(I)$ and restrict it to $C\left(N_{1}\right) \times C\left(N_{2}\right)$. The type-preserving condition implies uniqueness.

Lemma 3.7 (Links in the cone) Let $N$ be a simplicial complex, $w$ a vertex of the cubical cone $C(N)$, and $t(w)=J \subset \bar{N}$ the type of $w$.

Then a cube $C$ of $C(N)$ contains $w$ if and only if $t(w) \subset t(C)$.

In particular the link of $w$ in $C(N)$ is isomorphic to the union of simplices of $N$ containing $t(w)$ under the map assigning to each simplex $\sigma$ of $\operatorname{link}(w, C(N))$ the simplex $t(C(\sigma))$, where $C(\sigma)$ is the cube of $C(N)$ containing $w$ and corresponding to $\sigma$. 
Proof Indeed $w \in C \Longleftrightarrow Q(\{w\}) \subset Q(C)$.

Note that cubical cones on the boundary of simplices are the building blocks of a subdivision of simplicial complexes into cubical complexes. Thus topologically, cube complexes and simplicial complexes are the same.

The specificity of cube complexes appears as soon as geometry is involved. According to Gromov [16], there are very simple local conditions on a cube complex which make it a nonpositively curved space in the sense of Aleksandrov:

Theorem 3.8 Let $X$ denote any locally finite cube complex. Endow $X$ with the length metric inducing on each cube the unit Euclidean metric. Then $X$ is locally $\mathrm{CAT}(0)$ if and only if each vertex link of $X$ is a flag simplicial complex.

This justifies the following:

Definition 3.9 We say that a cube complex $X$ is nonpositively curved if for each vertex $v$ the complex $\operatorname{link}(v, X)$ is a flag simplicial complex. We say that $X$ is $\operatorname{CAT}(0)$ whenever it is simply connected and nonpositively curved.

For references on the CAT(0) inequality, see Bridson and Haefliger [8]. For local combinatorial conditions on simplicial complexes implying a nonpositively curved behaviour, see [21] and [29].

\section{The right-angled building of a graph product of finite groups}

All the material in this section is well-known to people working on graph products or right-angled buildings. For convenience we have written explicitly most of the arguments.

\subsection{Graph products}

Definition 4.1 (Graph products) Let $\mathcal{G}$ denote any simplicial graph with vertex set $I$. Suppose that for each $i \in I$ we are given a group $G_{i}$. We define the graph product of $\left\{G_{i}\right\}_{i \in I}$ along $\mathcal{G}$ to be the group $\Gamma=\Gamma\left(\mathcal{G},\left(G_{i}\right)_{i \in I}\right)$ defined as follows.

$\Gamma$ is the quotient of the free product $* G_{i}$ by the normal subgroup generated by all elements of the form $g_{i} g_{j} g_{i}^{-1} g_{j}^{-1}$, where $g_{i} \in G_{i}, g_{j} \in G_{j}$ for distinct adjacent vertices $i, j$ in $\mathcal{G}$. 
For any subset $J \subset I$ we denote by $\Gamma_{J}$ the subgroup of $\Gamma$ generated by the image of $\left\{G_{i}\right\}_{i \in I}$ under the natural morphism $G_{j} \rightarrow \Gamma$, and we denote by $\mathcal{G}_{J}$ the graph on $J$ induced by $\mathcal{G}$.

A subset $J \subset I$ is said to be spherical whenever any two distinct vertices of $J$ are adjacent in $\mathcal{G}$. In the sequel we denote by $N(\mathcal{G})$ the abstract simplicial complex on $I$ whose simplices are the nonempty spherical subsets $J \subset I$.

For any element $i \in I$ we will denote by $i^{\perp}$ the set of $j \in I \backslash\{i\}$ such that $j$ is adjacent to $i$ in $\mathcal{G}$. We also consider the subset $i^{\perp}=i^{\perp} \cup\{i\}$.

For example if $\mathcal{G}$ is a complete graph then $\Gamma$ is the direct product of the groups $G_{i}$, $i \in I$. In contrast, if $\mathcal{G}$ is totally disconnected then $\Gamma$ is the free product $*_{i \in I} G_{i}$.

Some references on graph products (and their buildings) are Green [15], Januszkiewicz and Świa̧tkowski [28], and Meier [33].

In the rest of the first part we assume we are given a graph $\mathcal{G}$ with vertex set $I$, together with a group $G_{i}$ for each $i \in I$. We denote by $\Gamma$ the graph product of $\left\{G_{i}\right\}_{i \in I}$ along $\mathcal{G}$.

The following result is proved in [28], for example.

Lemma 4.2 (Naturality) For any $i \in I$ the natural map $G_{i} \rightarrow \Gamma$ is injective. So we will identify $G_{i}$ and $\Gamma_{i}$. More generally for any subset $J \subset I$ the natural map from the free product $*_{j \in J} G_{j}$ to the group $\Gamma$ induces an isomorphism $\Gamma\left(\mathcal{G}_{J},\left(G_{i}\right)_{i \in J}\right) \rightarrow \Gamma_{J}$.

Proof Consider the morphism from the free product $*_{i \in I} G_{i}$ onto the free product $*_{j \in J} G_{j}$ that kills each $G_{k}, k \notin J$, and induces the identity on each $G_{j}, j \in J$. This morphism induces a morphism $\rho_{J}$ from $\Gamma\left(\mathcal{G},\left(G_{i}\right)_{i \in I}\right)$ to $\Gamma\left(\mathcal{G}_{J},\left(G_{i}\right)_{i \in J}\right)$. Now the natural map $\Gamma\left(\mathcal{G}_{J},\left(G_{i}\right)_{i \in J}\right) \rightarrow \Gamma\left(\mathcal{G},\left(G_{i}\right)_{i \in I}\right)$ postcomposed with $\rho_{J}$ is the identity.

\subsection{The building of a graph product}

Definition 4.3 Let $C=C(N(\mathcal{G})$ ) denote the cubical cone over $N(\mathcal{G})$ (see Lemma 3.5). Consider the equivalence relation on $\Gamma \times C$ defined by

$$
(\gamma, p) \sim\left(\gamma^{\prime}, p^{\prime}\right) \Longleftrightarrow p=p^{\prime} \in \partial C \text { and } \gamma^{-1} \gamma^{\prime} \in \Gamma_{J} \text { for } J=\underline{t}(p) .
$$

Set $\Delta=\Delta\left(\mathcal{G},\left(G_{i}\right)_{i \in I}\right)=\Gamma \times C / \sim$, and denote by $[\gamma, p]$ the equivalence class of $(\gamma, p)$.

For $\gamma \in \Gamma$, the image under the natural map $\pi: \Gamma \times C \rightarrow \Delta$ of the $\{\gamma\} \times C$ is called a chamber of $\Delta$, denoted by $C_{\gamma}$. We denote the base chamber $C_{1_{\Gamma}}$ by $C_{*}$. 
Lemma 4.4 Let $C$ be as in Definition 4.3. Then the following observations easily hold.

(1) The left action of $\Gamma$ on $\Gamma \times C$ induces an action by homeomorphisms on $\Delta$.

(2) The second projection $\Gamma \times C \rightarrow\left\{1_{\Gamma}\right\} \times C$ induces a topological retraction map $\rho: \Delta \rightarrow C_{*}$. The natural map $C \rightarrow C_{*}$ given by the inclusion $C \rightarrow\left\{1_{\Gamma}\right\} \times C$ postcomposed with $\pi$ is a homeomorphism. Thus we may identify $C$ with $C_{*}$.

(3) The map $\rho$ is invariant under $\Gamma$. In fact two points of $\Delta$ belong to the same $\Gamma$-orbit if and only if they have the same image under $\rho$.

(4) The base chamber $C_{*}$ is a strict fundamental domain for the action of $\Gamma$ on $\Delta$. So every $\Gamma$-orbit in $\Delta$ meets $C_{*}$ at a one and only one point.

The following is straightforward.

Lemma 4.5 Each building $\Delta$ admits a unique cube complex structure for which the natural map $\pi: \Gamma \times C \rightarrow \Delta$ is combinatorial.

We will always endow $\Delta$ with this structure.

Remark 4.6 Note that (1) chambers are subcomplexes, (2) $\Gamma$ acts as a group of combinatorial automorphisms and (3) the retraction $\rho: \Delta \rightarrow C$ is combinatorial.

\subsection{The typing map}

Definition 4.7 Recall from Lemma 3.5 that we have already defined a map $t$ assigning to each vertex of the cubical cone $C=C(N(\mathcal{G}))$ an element of $\overline{N(\mathcal{G})}$, ie, a simplex of $N(\mathcal{G})$ or the empty subset of $I$. This map is also defined on the set of vertices of $\Gamma \times C$ and induces a map $t: \Delta^{0} \rightarrow \overline{N(\mathcal{G})}$, called the typing map on $\Delta$. On the set of vertices of the base chamber $C_{*}$ identified with $C$, the map $t$ is the usual one. We define similarly for any cube $Q$ of $\Delta$ the elements $t(Q)$ or $\underline{t}(Q)$ by first taking the image $\rho(Q)$ inside $C_{*} \simeq C$ and then applying the usual maps $t$ or $\underline{t}$. Equivalently $t(Q)$ is the maximum of all $t(v)$ for $v$ a vertex of $Q$ and $\underline{t}(Q)$ is the minimum of all $t(v)$ for $v$ a vertex of $Q$.

The rank of a vertex $v$ is the cardinality $\operatorname{rk}(v)$ of $t(v)$. The rank-0 vertices will be called centers of chambers. We will denote the center of $C_{*}$ by $v_{*}$.

We will denote by $\operatorname{Aut}_{0}(\Delta)$ the subgroup of the group of combinatorial automorphisms of $\Delta$ whose elements preserve the typing map $t$. Note that $t$ is invariant under $\Gamma$, ie, $\left.\Gamma \subset \operatorname{Aut}_{0}(\Delta)\right)$ and $\rho$. 
Lemma 4.8 For any $J \in \overline{N(\mathcal{G})}$, each chamber contains a single vertex $v$ of type $t(v)=J$. The stabilizer of the unique vertex of type $J$ in $C_{*}$ is the group $\Gamma_{J}$. The stabilizer of a vertex acts simply transitively on the set of chambers containing this vertex.

In particular $\Gamma$ is simply transitive on centers of chambers and on chambers.

This implies that the action of $\Gamma$ on $\Delta$ is faithful. Hence we will identify $\Gamma$ with its image in $\operatorname{Aut}_{0}(\Delta)$.

Proof Using the $\Gamma$ invariance of $t$, it suffices to prove the first assertion for the base chamber $C_{*}$.

Let $v_{J}$ denote the unique vertex of $C$ with $t\left(v_{J}\right)=J$ (see Lemma 3.5). Then [1, $\left.v_{J}\right]$ is the unique vertex of $C_{*}$ with type $J$. Now $\gamma\left[1, v_{J}\right]=\left[1, v_{J}\right]$ if and only if $\gamma \in \Gamma_{J}$, by definition of $\Delta$.

For $J=\varnothing$, we have $\Gamma_{J}=\{1\}$, thus $\Gamma$ acts freely on centers of chambers (by construction it acts transitively).

In particular if $\gamma C_{*}=C_{*}$ then $\gamma$ fixes the center of $C_{*}$ (we have just seen that there is a unique rank-0 vertex in each chamber). Thus $\gamma=1$. The simple transitivity of $\Gamma$ on the set of chambers follows.

Let us check now that $\Gamma_{J}$ acts simply transitively on the set of chambers containing $v_{J}$. The action is free because $\Gamma$ acts freely on the set of chambers. Let $C=\gamma C_{*}$ denote a chamber containing $v_{J}$. Then $\gamma v_{J}$ is a vertex of type $J$ inside $C$. By the first part of the lemma, we have $\gamma v_{J}=v_{J}$. Thus $\gamma \in \Gamma_{J}$ as claimed.

For each vertex $v$ of $\Delta$ we denote by $\mathcal{C}(v)$ the set of chambers of $\Delta$ containing $v$. This set is in one-to-one correspondence with the stabilizer of $v$ in $\Gamma$ by the previous lemma. As a consequence we obtain the following:

Corollary 4.9 For each vertex $v$ of $\Delta$ we have $\operatorname{link}(v, \Delta)=\bigcup_{C \in \mathcal{C}(v)} \operatorname{link}(v, C)$.

In particular, the building $\Delta$ is locally compact if and only if the graph $\mathcal{G}$ is finite and each generating group $G_{i}$ is finite.

\subsection{Residues}

Definition 4.10 (Chamber system and residues) Let $\mathcal{C}$ denote the set of chambers of $\Delta$. Note that $\mathcal{C}$ is identified under $\gamma \mapsto C_{\gamma}$ with $\Gamma$ (see Lemma 4.8). We follow Ronan [36] to define on the set $\mathcal{C}$ a structure of chamber system over $I$. For each $i \in I$ we 
say that two chambers $C_{1}, C_{2}$ of $\Delta$ are $i$-adjacent (written $C_{1} \sim_{i} C_{2}$ ) whenever the intersection $C_{1} \cap C_{2}$ contains a vertex $v$ with $t(v)=\{i\}$.

We then define for any subset $J \subset I$ the equivalence relation $\sim_{J}$ on $\mathcal{C}$ as the equivalence relation generated by $\sim_{j}$, for $j \in J$ (by convention $\sim_{\varnothing}$ is the equivalence relation whose orbits are the singletons). We define the extended $J$-residue of a chamber $C$ to be the union of the chambers $C^{\prime}$ for which $C^{\prime} \sim{ }_{J} C$. We will denote this subcomplex by $\bar{R}(J, C)$ (or sometimes $\bar{R}_{\Delta}(J, C)$ ). For example $\bar{R}(\varnothing, C)=C$.

Now we define the $J$-residue of a chamber $C$ as the union of cubes $Q$ of $\bar{R}(J, C)$ such that $t(Q) \subset J$; we denote it by $R(J, C)$ (or sometimes $R_{\Delta}(J, C)$ ). For example, $R(\varnothing, C)=\{v\}$ where $v$ is the center of the chamber $C$.

For any residue $R$ of $\Delta$ we will denote by $\mathcal{C}(R)$ the set of chambers of $\Delta$ whose center is contained in $R$.

Clearly for any $g \in \operatorname{Aut}_{0}(\Delta)$ each $i$-adjacency is invariant under $g$. Thus for any subset $J \subset I$ the relation $\sim_{J}$ is also invariant under $g$, and for any chamber $C$ we have $g R(J, C)=R(J, g C)$. For example we have $R\left(J, C_{\gamma}\right)=\gamma R\left(J, C_{*}\right)$.

Note that extended residues are unions of chambers, while for $J \neq I$, no $J$-residue contains a chamber.
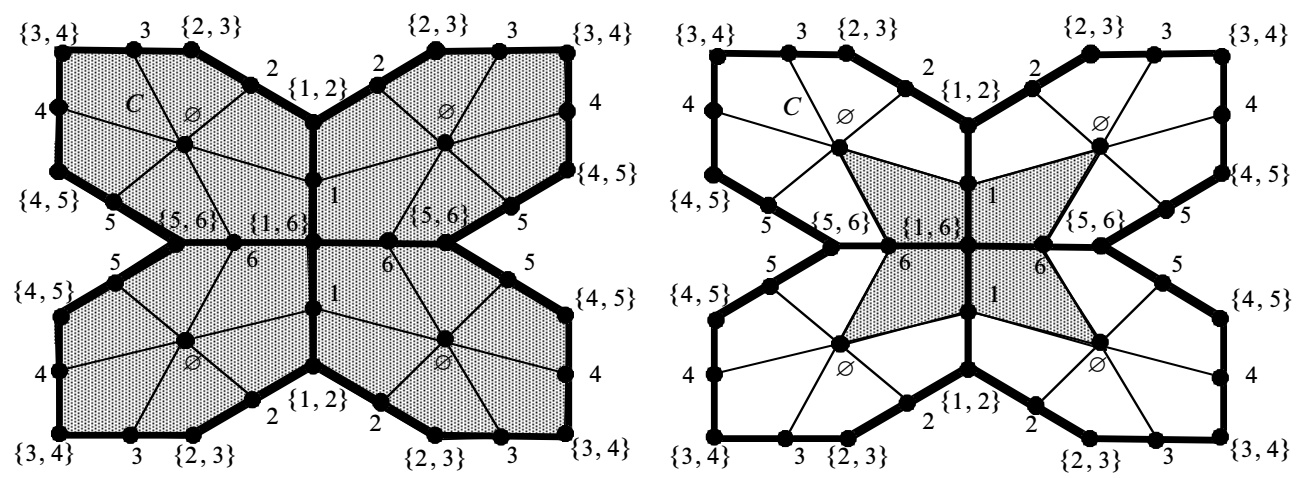

Figure 2: Left: The extended $\{1,6\}$-residue of a chamber C. Right: The $\{1,6\}$-residue of the same chamber. In both cases the building is Fuchsian: it corresponds to the circular graph on $I=\{1,2,3,4,5,6\}$ when the groups $G_{i}$ are of order two.

Lemma 4.11 Let $i$ denote some element of $I$ and let $C$ and $C^{\prime}$ be any pair of distinct $i$-adjacent chambers. Then either $C=C^{\prime}$ or $C \cap C^{\prime}$ contains a single rank-1 vertex. 
Proof It is enough to argue when $C=C_{*}$. By Lemma 4.8, $C \cap C^{\prime}$ contains a single vertex $w$ of type $\{i\}$. Assume that $C \cap C^{\prime}$ contains a vertex $w^{\prime}$ of type $\{j\}$ with $j \neq i$.

Then by Lemma 4.8, there are $\gamma \in \Gamma_{i}$ and $\gamma^{\prime} \in \Gamma_{j}$ such that $C^{\prime}=\gamma C$ and $C^{\prime}=\gamma^{\prime} C$. Since $\Gamma$ acts simply transitively on chambers we have $\gamma=\gamma^{\prime}$.

So the lemma will follow if we prove that $\Gamma_{i} \cap \Gamma_{j}=\{1\}$ whenever $i \neq j$. But this relation is true when the set of vertices of $\mathcal{G}$ is $\{i, j\}$, hence it is true in general because $\Gamma_{\{i, j\}}$ embeds in $\Gamma$ by Lemma 4.2.

Lemma 4.12 Let $R=R(J, C)$ denote some residue of $\Delta$. Let $C^{\prime}$ denote a chamber of center $w^{\prime}$. Then $w^{\prime} \in R$ if and only if $C \sim{ }_{J} C^{\prime}$. Equivalently $C^{\prime} \in \mathcal{C}(R) \Longleftrightarrow$ $C^{\prime} \sim{ }_{J} C$.

Proof The "if" part follows by definition. Conversely assume $w^{\prime} \in R$. Then $w^{\prime}$ lies in some chamber $C^{\prime \prime}$ such that $C^{\prime \prime} \sim_{J} C$. By Lemma $4.8, w^{\prime}$ has to be the center of $C^{\prime \prime}$, hence $C^{\prime \prime}=C^{\prime}$ and we are done.

Definition 4.13 (Galleries) A gallery of $\Delta$ of length $n$ joining $C$ and $C^{\prime}$ is a sequence of chambers $\left(C_{0}, C_{1}, \ldots, C_{n}\right)$ such that $C_{0}=C, C_{n}=C^{\prime}$ and for each integer $1 \leq k \leq n$ the chambers $C_{k-1}$ and $C_{k}$ are $i_{k}$-adjacent for some $i_{k} \in I$. We say that $\left(C_{0}, C_{1}, \ldots, C_{n}\right)$ is a $J$-gallery whenever for each integer $1 \leq k \leq n$ the chambers $C_{k-1}$ and $C_{k}$ are $j_{k}$-adjacent for some $j_{k} \in J$. A gallery $\left(C_{0}, C_{1}, \ldots, C_{n}\right)$ is closed whenever $C_{0}=C_{n}$.

The product of two galleries $G \cdot G^{\prime}$ is defined by concatenation.

The combinatorial distance between two chambers is the minimal length of a gallery joining them. A gallery is said to be geodesic whenever its length is the distance between its endpoints.

Lemma 4.14 (Residues are subbuildings) Let $J$ denote any subset of $I$. The natural maps $\Gamma\left(\mathcal{G}_{J},\left(G_{i}\right)_{i \in J}\right) \rightarrow \Gamma_{J} \subset \Gamma=\Gamma\left(\mathcal{G},\left(G_{i}\right)_{i \in I}\right)$ and $C\left(N\left(\mathcal{G}_{J}\right)\right) \rightarrow C(N(\mathcal{G}))$ induce an equivariant type-preserving combinatorial embedding $\delta_{J}$ from $\Delta\left(\mathcal{G}_{J},\left(G_{i}\right)_{i \in J}\right)$ to $\Delta\left(\mathcal{G},\left(G_{i}\right)_{i \in I}\right)$ whose image is the residue $R\left(J, C_{*}\right)$.

Proof First, the inclusion $\mathcal{G}_{J} \subset \mathcal{G}$ yields a natural combinatorial inclusion of $N\left(\mathcal{G}_{J}\right)$ into $N(\mathcal{G})$. Then there is an induced combinatorial map $i_{J}: C\left(N\left(\mathcal{G}_{J}\right)\right) \rightarrow C(N(\mathcal{G}))$, by Lemma 3.5. If two vertices $x$ and $y$ are identified under this map then the unique smallest cubes $Q_{x}$ and $Q_{y}$ containing $\{x, v\}$ and $\{y, v\}$ are mapped to the same cube. By injectivity of the map $N\left(\mathcal{G}_{J}\right) \rightarrow N(\mathcal{G})$ this means that $Q_{x}=Q_{y}$, whence $x=y$. 
Now the injectivity of $C\left(N\left(\mathcal{G}_{J}\right)\right) \rightarrow C(N(\mathcal{G}))$ on the set of vertices implies injectivity because cubical cones are simple cube complexes.

Note that the inclusion $C\left(N\left(\mathcal{G}_{J}\right)\right) \rightarrow C(N(\mathcal{G}))$ preserves the typing maps.

We already know that the natural morphism $\Gamma\left(\mathcal{G}_{J},\left(G_{i}\right)_{i \in J}\right) \rightarrow \Gamma_{J}$ is an isomorphism. Consider the map $\Gamma\left(\mathcal{G}_{J},\left(G_{i}\right)_{i \in J}\right) \times C\left(N\left(\mathcal{G}_{J}\right)\right) \rightarrow \Gamma \times C(N(\mathcal{G}))$. It is a type-preserving, equivariant inclusion, and it induces a type-preserving, equivariant combinatorial map $\delta_{J}: \Delta\left(\mathcal{G}_{J},\left(G_{i}\right)_{i \in J}\right) \rightarrow \Delta$. Now assume two pairs $(\gamma, p)$ and $\left(\gamma^{\prime}, p^{\prime}\right)$ of $\Gamma\left(\mathcal{G}_{J},\left(G_{i}\right)_{i \in J}\right) \times C\left(N\left(\mathcal{G}_{J}\right)\right)$ are identified in $\Delta$. This means that $i_{J}(p)=i_{J}\left(p^{\prime}\right)$ and that $\gamma^{-1} \gamma^{\prime} \in \Gamma_{\underline{t}\left(i_{J}(p)\right)}$. Using injectivity of the natural maps we see that in fact $(\gamma, p)$ and $\left(\gamma^{\prime}, p^{\prime}\right)$ are identified in $\Delta\left(\mathcal{G}_{J},\left(G_{i}\right)_{i \in J}\right)$.

It remains to show that the image of $\delta_{J}$ is $R\left(J, C_{*}\right)$. First, the image of the base chamber $C_{*}^{J}$ of $\Delta\left(\mathcal{G}_{J},\left(G_{i}\right)_{i \in J}\right)$ is the union of cubes $Q$ of $C_{*}$ containing the center $v_{*}$ of $C_{*}$ and such that (either $Q=\left\{v_{*}\right\}$ or) $\operatorname{link}\left(v_{*}, Q\right)$ is a simplex of $N\left(\mathcal{G}_{J}\right)$. The last condition means that the types of the vertices of $Q$ are simplices of $N$ whose vertices are in $J$, thus $t(Q) \subset J$. So we have shown that $C_{*}^{J}$ maps into $C_{*} \cap R\left(J, C_{*}\right)$.

In fact any cube $Q$ of $C_{*} \cap R\left(J, C_{*}\right)$ corresponds to the image of a cube of $C_{*}^{J}$. For $t(Q) \subset J$ and $t(Q)$ is a simplex of $N(\mathcal{G})$ since its vertices are pairwise adjacent in $\mathcal{G}$. So the vertices of $t(Q)$ are pairwise adjacent in $\mathcal{G}_{J}$, and $t(Q) \in N\left(\mathcal{G}_{J}\right)$. This implies that $Q \in \delta_{J}\left(C_{*}^{J}\right)$.

Let us check that $R\left(J, C_{*}\right)$ is invariant under $\Gamma_{J}$. As $\Gamma_{J}$ is generated by $\Gamma_{j}=G_{j}$ for $j \in J$, it enough to prove that $R\left(J, C_{*}\right)$ is invariant under $\Gamma_{j}, j \in J$. Now for $\gamma \in \Gamma_{j}$ we have $\gamma \bar{R}\left(J, C_{*}\right)=\bar{R}\left(J, \gamma C_{*}\right)$, and $\gamma C_{*} \cap C_{*}$ contains the unique vertex of $C_{*}$ of type $\{j\}$. Thus $\gamma C_{*} \sim_{j} C_{*}$ and $\gamma \bar{R}\left(J, C_{*}\right)=\bar{R}\left(J, C_{*}\right)$. It follows that $\gamma R\left(J, C_{*}\right)=R\left(J, C_{*}\right)$.

At this stage we know the following: $C_{*}^{J}$ is mapped into $R\left(J, C_{*}\right)$, the building $\Delta\left(\mathcal{G}_{J},\left(G_{i}\right)_{i \in J}\right)$ is the union of the $\Gamma\left(\mathcal{G}_{J},\left(G_{i}\right)_{i \in J}\right)$-translates of $C_{*}^{J}$, the combinatorial map $\delta_{J}$ is equivariant, and $R\left(J, C_{*}\right)$ is invariant under $\Gamma_{J}$. This implies that the image of $\delta_{J}$ is contained in $R\left(J, C_{*}\right)$. It remains to prove the reverse inclusion.

Let $C$ denote any chamber such that $C \sim{ }_{J} C_{*}$. So there is a sequence of elements $\gamma_{1}, \ldots, \gamma_{n}$ of $\Gamma$ such that $\left(C_{*}, C_{\gamma_{1}}, \ldots, C_{\gamma_{n}}\right)$ is a $J$-gallery and $C_{\gamma_{n}}=C$. By induction on $n$ we may assume that $\gamma_{i} \in \Gamma_{J}$ for each $i<n$. Now by definition $C_{\gamma_{n}}$ is $j$-adjacent to $C_{\gamma_{n-1}}$ for some $j \in J$. Applying $\gamma_{n-1}^{-1}$ shows that $C_{\gamma_{n-1}^{-1} \gamma_{n}}$ is $j$-adjacent to $C_{*}$.

So $\gamma_{n-1}^{-1} \gamma_{n} C_{*} \cap C_{*}$ contains the unique vertex $v_{*}^{j}$ of $C_{*}$ such that $t\left(v_{*}^{j}\right)=j$. As $C_{*}$ is a strict fundamental domain this implies $\gamma_{n-1}^{-1} \gamma_{n} v_{*}^{j}=v_{*}^{j}$. By Lemma 4.8, we then have $\gamma_{n-1}^{-1} \gamma_{n} \in \Gamma_{j}$, and thus $\gamma_{n} \in \Gamma_{J}$. 
This implies that $\bar{R}\left(J, C_{*}\right)=\Gamma_{J} C_{*}$. Thus $R\left(J, C_{*}\right)=\Gamma_{J}\left(C_{*} \cap R\left(J, C_{*}\right)\right)$. As we have already seen, $C_{*} \cap R\left(J, C_{*}\right)=\delta_{J}\left(C_{*}^{J}\right)$. We deduce that the image of $\delta_{J}$ is the full residue $R\left(J, C_{*}\right)$.

Each residue $R$ of $\Delta$ is isomorphic to a right-angled building, thus we will denote by $\operatorname{Aut}_{0}(R)$ the group of those combinatorial automorphisms of the cube complex $R$ preserving the restriction of the typing map $t$ on the set of vertices of $R$.

Corollary 4.15 Let $\gamma \in \Gamma$ and let $J \subset I$. The chambers $C$ such that $C \sim_{J} C_{\gamma}$ are the chambers $C_{\gamma^{\prime}}$ with $\gamma^{\prime} \in \gamma \Gamma_{J}$. The stabilizer of $R\left(J, C_{\gamma}\right)$ in $\Gamma$ is the conjugate $\gamma \Gamma_{J} \gamma^{-1}$. It acts simply transitively on $\mathcal{C}\left(R\left(J, C_{\gamma}\right)\right)$.

Proof By transitivity of $\Gamma$ on the set of chambers of $\Delta$ it suffices to argue when $\gamma=1$.

By Lemma 4.8, the graph product $\Gamma\left(\mathcal{G}_{J},\left(G_{i}\right)_{i \in J}\right)$ acts simply transitively on the rank-0 vertices of $\Delta\left(\mathcal{G}_{J},\left(G_{i}\right)_{i \in J}\right)$. By Lemma 4.14 , we deduce that $\Gamma_{J}$ preserves $R\left(J, C_{*}\right)$ and acts simply transitively on the rank-0 vertices of $R\left(J, C_{*}\right)$.

Let $C$ denote a chamber with center $\gamma^{\prime} v_{*}$. Then by Lemma 4.12 we have $C \sim_{J} C_{*}$ if and only if $\gamma^{\prime} v_{*} \in R\left(J, C_{*}\right)$. This is equivalent to the fact that there exists a $\gamma^{\prime \prime} \in \Gamma_{J}$ such that $\gamma^{\prime} v_{*}=\gamma^{\prime \prime} v_{*}$. Applying Lemma 4.8 now in $\Delta$, we see that $\gamma^{\prime} v_{*} \in R\left(J, C_{*}\right)$ is equivalent to $\gamma^{\prime} \in \Gamma_{J}$.

This proves that the chambers $C$ such that $C \sim{ }_{J} C_{*}$ are the chambers $C_{\gamma^{\prime}}$ with $\gamma^{\prime} \in \Gamma_{J}$. The rest of the corollary follows easily.

\subsection{Local structure and simple connectedness of the building}

Definition 4.16 (Lower link) For any vertex $v$ of $\Delta$ let $\underline{\operatorname{link}}(v, \Delta)$ denote the subcomplex of $\operatorname{link}(v, \Delta)$ whose simplices correspond to cubes $Q$ containing $v$ and such that $t(Q) \subset t(v)$. We call this subcomplex of the link the lower link of $v$.

Lemma 4.17 Let $v$ denote any vertex of $\Delta$. Then $\underline{\operatorname{link}}(v, \Delta)$ is a thickened octahedron. Each maximal simplex of the lower link is a strict fundamental domain for the action of the stabilizer of $v$ in $\Gamma$.

Proof As usual we may assume that $v=v_{*}$. Let $J \in \bar{N}$ denote the type of $v$. Then $\underline{\operatorname{link}}(v, \Delta)=\operatorname{link}\left(v, R\left(J, C_{*}\right)\right)$. Now the base chamber is always a strict fundamental domain for the action of the graph product. Thus the cube $Q_{v}=C_{*} \cap R\left(J, C_{*}\right)$ is a strict fundamental domain for the action of $\Gamma_{J}$, and the $\operatorname{simplex} \operatorname{link}\left(v, Q_{v}\right)$ is a strict fundamental domain for the action of $\Gamma_{J}$ on $\underline{\operatorname{link}}(v, \Delta)$. Now Example 3.3 concludes the proof.

Algebraic $8 \mathcal{G}$ Geometric Topology, Volume 6 (2006) 
Lemma 4.18 (Links in $\Delta$ ) Let $v$ denote a vertex of $C_{*}$ of type $t(v)=J \subset \bar{N}$. Let $\varlimsup \overline{\operatorname{link}}(v, \Delta) \subset \operatorname{link}(v, \Delta)$ denote the union of simplices $\sigma$ corresponding to cubes $Q$ such that the vertex $v \in Q \subset C_{*}$, the intersection $t(Q) \cap J$ is empty and the union $t(Q) J$ is a simplex of $N$. (In other words, $\overline{\operatorname{link}}(v, \Delta)$ corresponds to the full subcomplex of $N$ on vertices which are joinable in $\mathcal{G}$ to each vertex of $J$.) Then the link of $v$ in $\Delta$ is the join of $\overline{\operatorname{link}}(v, \Delta)$ with $\underline{\operatorname{link}}(v, \Delta)$.

Proof By Lemma 4.8, the group $\Gamma_{J}$ acts simply transitively on the set $\mathcal{C}(v)$ of chambers containing $v$. We have $\operatorname{link}(v, \Delta)=\bigcup_{C \in \mathcal{C}(v)} \operatorname{link}(v, C)$ by Corollary 4.9. Observe that $\Gamma_{J}$ fixes pointwise $\overline{\operatorname{link}}(v, \Delta)$. Thus the result follows because by Lemma 3.7, we have already $\operatorname{link}\left(v, C_{*}\right)=\varlimsup \operatorname{link}\left(v, C_{*}\right) * \underline{\operatorname{link}}\left(v, C_{*}\right)$.

Proposition 4.19 The building $\Delta$ is a CAT( 0$)$ cube complex. Residues of $\Delta$ are convex subcomplexes.

The fact that $\Delta$ is CAT(0) is proved by Davis [12] and Meier [33].

Proof By Lemma 4.18, we have a description of the link of each vertex $v$ as the join of two simplicial complexes $\underline{\operatorname{link}}(v, \Delta)$ and $\overline{\operatorname{link}}(v, \Delta)$. By Lemma 4.17, $\underline{\operatorname{link}}(v, \Delta)$ is a thickened octahedron, hence is a flag. By construction $\overline{\operatorname{link}}(v, \Delta)$ is the link of the simplex $t(v)$ in the flag complex $N$, thus it is a flag. Consequently each vertex link is a flag and $\Delta$ is nonpositively curved.

Let $\tilde{p}: \widetilde{\Delta} \rightarrow \Delta$ denote the universal cover. This induces a typing map and a notion of rank on the set of vertices of $\widetilde{\Delta}$. Let $\widetilde{C_{*}}$ denote some connected component of $p^{-1}\left(C_{*}\right)$. Since $C_{*}$ is a cone it is contractible, hence $p: \widetilde{C_{*}} \rightarrow C_{*}$ is an isomorphism. Let $\widetilde{\Gamma}$ denote the group of automorphisms $\tilde{\gamma}$ of $\widetilde{\Delta}$ such that there exists $\gamma \in \Gamma$ satisfying $p \circ \tilde{\gamma}=\gamma \circ p$. The map $\varphi: \tilde{\gamma} \mapsto \gamma$ is a morphism onto $\Gamma$; the kernel is the fundamental group of $\Delta$.

The subgroup $\Gamma_{i}=G_{i}$ is the stabilizer of the unique vertex $v_{*}^{i}$ of $C_{*}$ of type $\{i\}$. Let us denote by $\widetilde{\Gamma_{i}}$ the set of lifts of elements of $\Gamma_{i}$ fixing the unique vertex of $\widetilde{C_{*}}$ projecting to $v_{*}^{i}$. Then $\widetilde{\Gamma_{i}}$ is a subgroup of $\widetilde{\Gamma}$ for each $i \in I$, and the restriction $\varphi_{i}: \widetilde{\Gamma_{i}} \rightarrow \Gamma_{i}$ is an isomorphism.

Assume that $i$ and $j$ are linked in $\mathcal{G}$. Let $\tilde{v}$ denote the unique vertex of $\widetilde{C_{*}}$ projecting to the vertex $v$ of $C_{*}$ such that $t(v)=\{i, j\}$. Then for any $\widetilde{\gamma}_{i} \in \widetilde{\Gamma_{i}}$ and $\widetilde{\gamma}_{j} \in \widetilde{\Gamma_{j}}$, the commutator $\left[\tilde{\gamma}_{i}, \tilde{\gamma}_{j}\right]$ maps to $1 \in \Gamma$. Thus $\left[\tilde{\gamma}_{i}, \tilde{\gamma}_{j}\right]$ is in the deck transformation group. But it fixes $\widetilde{v}$. Thus $\left[\widetilde{\gamma}_{i}, \tilde{\gamma}_{j}\right]=1$. We have proved that $\left[\widetilde{\Gamma_{i}}, \widetilde{\Gamma_{j}}\right]=1$.

For each rank-0 vertex $v$ of $\Delta$, there is a path $\left(v_{0}=v_{*}, v_{1}, \ldots, v_{n}=v\right)$ using only vertices of rank at most 1 . The lift of this path at the center of $\widetilde{C_{*}}$ is contained in the 
orbit of $\widetilde{C_{*}}$ under the subgroup generated by $\left\{\widetilde{\Gamma_{i}}\right\}_{i \in I}$. The endpoint of this path is an arbitrary vertex projecting onto a rank-0 vertex. This shows that $\widetilde{\Gamma}$ is generated by $\left\{\widetilde{\Gamma_{i}}\right\}_{i \in I}$, because $\widetilde{\Gamma}$ acts freely on rank-0 vertices of $\widetilde{\Delta}$.

Now the inverse isomorphisms $\varphi_{i}^{-1}: \Gamma_{i} \rightarrow \widetilde{\Gamma}_{i}$ define a morphism $\psi: \Gamma \rightarrow \widetilde{\Gamma}$. We have $\psi \varphi=1$ on $\widetilde{\Gamma}$ because this is true on each $\widetilde{\Gamma_{i}}$, and these latter subgroups generate $\widetilde{\Gamma}$.

Thus $\operatorname{Ker} \varphi=1$ and $\Delta$ is simply connected, therefore $\operatorname{CAT}(0)$.

Now let $R$ denote some $J$-residue of $\Delta$, and let us prove that $R$ is CAT(0)-convex. We may assume that $R=R\left(J, C_{*}\right)$. Recall that there is an injective combinatorial map $i_{J}: C\left(N\left(\mathcal{G}_{J}\right)\right) \rightarrow C(N(\mathcal{G}))$ (see Lemma 4.14). But in fact there is also a cellular map $r_{J}: C(N(\mathcal{G})) \rightarrow C\left(N\left(\mathcal{G}_{J}\right)\right)$ such that $r_{J} \circ i_{J}=1:$ this is just the restriction to $C(N(\mathcal{G})) \subset \ell^{2}(I)$ of the orthogonal projection $\ell^{2}(I) \rightarrow \ell^{2}(J)$. Note that for any segment $[x, y]$ inside a cube of $C(N(\mathcal{G}))$ the length of $\left[r_{J}(x), r_{J}(y)\right]$ is not larger than that of $[x, y]$.

Recall that we also have a retraction morphism $\rho_{J}: \Gamma \rightarrow \Gamma\left(\mathcal{G}_{J},\left(G_{i}\right)_{i \in J}\right)$, such that $\rho_{J}$ composed with the natural inclusion $\Gamma\left(\mathcal{G}_{J},\left(G_{i}\right)_{i \in J}\right) \rightarrow \Gamma_{J}$ is the identity.

We leave it to the reader to verify that $\left(\rho_{J}, r_{J}\right): \Gamma \times C \rightarrow \Gamma\left(\mathcal{G}_{J},\left(G_{i}\right)_{i \in J}\right) \times C\left(N\left(\mathcal{G}_{J}\right)\right)$ induces a cellular map $\pi_{J}: \Delta \rightarrow \Delta\left(\mathcal{G}_{J},\left(G_{i}\right)_{i \in J}\right)$ such that $\pi_{J} \circ \delta_{J}=1$. In fact $\pi_{J}$ sends piecewise geodesics of length $L$ to piecewise geodesics of length $\leq L$.

Now take two points $p$ and $q$ of $R=R\left(J, C_{*}\right)=\delta_{J}\left(\Delta\left(\mathcal{G}_{J},\left(G_{i}\right)_{i \in J}\right)\right)$, and consider the geodesic $g$ between them. It follows that $g^{\prime}=\delta_{J}\left(\pi_{J}(g)\right)$ is a piecewise geodesic with endpoints $p$ and $q$ contained in $R$, and the length of $g^{\prime}$ is at most the length of $g$. Thus $g^{\prime}=g$ and $R$ is convex.

Definition 4.20 Two galleries $G=\left(C_{0}, \ldots, C_{n}\right)$ and $G^{\prime}=\left(C_{0}^{\prime}, \ldots, C_{m}^{\prime}\right)$ differ by an elementary (rank-r)-homotopy whenever there exists integers $i, j, k$ such that

(1) $\left(C_{0}, \ldots, C_{i}\right)=\left(C_{0}^{\prime}, \ldots, C_{i}^{\prime}\right)$

(2) the centers of $C_{i}, \ldots, C_{j}, C_{i}^{\prime}, \ldots, C_{k}^{\prime}$ belong to some residue $R(J, C)$ with $|J| \leq r$

(3) $\left(C_{j}, \ldots, C_{n}\right)=\left(C_{k}^{\prime}, \ldots, C_{m}^{\prime}\right)$.

The (rank-r)-homotopy is the equivalence relation (on galleries) generated by elementary (rank-r)-homotopies.

A lassoe is a closed gallery $G$ of the form $G^{\prime} \cdot\left(C_{0}, C_{1}, C_{2}, C_{3}, C_{0}\right) \cdot \overline{G^{\prime}}$, where $G^{\prime}$ denotes any gallery from the origin of $G$ to the chamber $C_{0}$, and $C_{0}, C_{1}, C_{2}, C_{3}$ are pairwise distinct chambers in some residue $R(J, C)$ with $J=\{i, j\}$ an edge of $\mathcal{G}$. 
Lemma 4.21 Every closed gallery of $\Delta$ is (rank-1)-homotopic to a product of lassoes.

Proof Let $G=\left(C_{0}, C_{1}, \ldots, C_{n}\right)$ denote a closed gallery. Using transitivity we may assume $C_{0}=C_{n}=C_{*}$. Let $\gamma_{j} \in \Gamma$ be such that $C_{j}=\gamma_{j} C_{*}$. Set $g_{j}=\gamma_{j}^{-1} \gamma_{j+1}$. Since $C_{j-1}$ and $C_{j}$ are adjacent chambers, either $C_{j-1}=C_{j}$ in which case we have $g_{j}=1$, or there exists a unique $i_{j} \in I$ such that $g_{j} \in G_{i_{j}}$. Thus we obtain a sequence $g_{1}, \ldots, g_{n}$ of $\bigcup_{i} G_{i} \subset \Gamma$ such that $C_{j}=g_{1} \ldots g_{j} C_{*}$ and $g_{1} \ldots g_{n}=1$.

This means that in the free product $*_{i \in I} G_{i}$, the element $g_{1} \ldots g_{n}$ is a product of conjugate of commutators $\left[h_{k}, h_{\ell}\right]$, with $h_{k} \in G_{k} \backslash\{1\}$ and $h_{\ell} \in G_{\ell} \backslash\{1\}, k \neq \ell$. Note that two products $a_{1} \ldots a_{p}$ and $b_{1} \ldots b_{q}$ for $a_{i}, b_{j} \in \bigcup_{k} G_{k}$ are equal in the free product $* G_{k}$ if and only if the corresponding galleries $\left(C_{*}, a_{1} C_{*}, \ldots, a_{1} \ldots a_{p} C_{*}\right)$ and $\left(C_{*}, b_{1} C_{*}, \ldots, b_{1} \ldots b_{q} C_{*}\right)$ are (rank-1)-homotopic.

This shows that our initial gallery $G$ is (rank-1)-homotopic to a product of lassoes, since the gallery with initial chamber $C_{*}$ corresponding to a conjugate of a commutator $\left[h_{k}, h_{\ell}\right]$ as above is a lassoe.

\subsection{Product structure of certain buildings and residues}

Lemma 4.22 Let $\mathcal{G}$ denote a graph with vertex set $I$. Let $\left(G_{i}\right)_{i \in I}$ denote a family of groups. Assume that we are given a partition $I=I_{1} \sqcup I_{2}$ in such a way that each $i_{1} \in I_{1}$ is adjacent to each $i_{2} \in I_{2}$. Consider the three buildings

$$
\Delta=\Delta\left(\mathcal{G},\left(G_{i}\right)_{i \in I}\right), \quad \Delta_{1}=\Delta\left(\mathcal{G}_{I_{1}},\left(G_{i}\right)_{i \in I_{1}}\right), \quad \text { and } \quad \Delta_{2}=\Delta\left(\mathcal{G}_{I_{2}},\left(G_{i}\right)_{i \in I_{2}}\right) .
$$

Then there exists an equivariant isomorphism of cube complexes $\delta: \Delta_{1} \times \Delta_{2} \rightarrow \Delta$ such that $\delta\left(p, v_{*}^{I_{2}}\right)=\delta_{I_{1}}(p), \delta\left(v_{*}^{I_{1}}, p\right)=\delta_{I_{2}}(p)$ and $t(\delta(p, q))=t_{1}(p) * t_{2}(q)$, where we denote by $\tau_{1} * \tau_{2}$ the simplex generated by $\tau_{1} \cup \tau_{2}$. Such an isomorphism is unique.

Proof Denote by $\Gamma, \Gamma_{1}$ and $\Gamma_{2}$ the graph products associated to the graphs $\mathcal{G}, \mathcal{G}_{I_{1}}$ and $\mathcal{G}_{I_{2}}$, respectively. By definition of graph product, there is a natural isomorphism $\Gamma_{1} \times \Gamma_{2} \rightarrow \Gamma$ (on the generating groups $G_{i_{1}} \times\left\{1_{\Gamma_{2}}\right\}$ it forgets the second component and acts similarly on the generating groups $\left\{1_{\Gamma_{1}}\right\} \times G_{i_{2}}$ ).

Let us check now that the chamber $C_{*}$ is naturally the product $C_{*}^{I_{1}} \times C_{*}^{I_{2}}$. To see this note that by assumption the simplicial complex $N=N(\mathcal{G})$ is the join of its subcomplexes $N_{1}=N\left(\mathcal{G}_{I_{1}}\right)$ and $N_{2}=N\left(\mathcal{G}_{I_{2}}\right)$. By Lemma 3.6 there is a typepreserving isomorphism $j: C\left(N_{1}\right) \times C\left(N_{2}\right) \rightarrow C(N)$.

Now combining the two isomorphisms we get an equivariant type-preserving isomorphism $\left(\Gamma_{1} \times \Gamma_{2}\right) \times\left(C\left(N_{1}\right) \times C\left(N_{2}\right)\right) \rightarrow \Gamma \times C(N)$. If we interpret the domain 
$\left(\Gamma_{1} \times \Gamma_{2}\right) \times\left(C\left(N_{1}\right) \times C\left(N_{2}\right)\right)$ as $\left(\Gamma_{1} \times C\left(N_{1}\right)\right) \times\left(\Gamma_{2} \times C\left(N_{2}\right)\right)$ it is routine to check that the isomorphism induces an equivariant type-preserving isomorphism $\delta: \Delta_{1} \times \Delta_{2} \rightarrow \Delta$ sending the base point $\left(v_{*}^{I_{1}}, v_{*}^{I_{2}}\right)$ to the base point $v_{*}$.

Two isomorphisms with the properties above would differ by a type-preserving automorphism $\varphi$ of $\Delta$ commuting with $\Gamma$ and fixing $v_{*}$. We know that $\varphi\left(v_{*}\right)=v_{*}$, $\varphi\left(C_{*}\right)=C_{*}$ and $\varphi$ preserves the type. But by Lemma 4.8 the typing map $t$ realizes a bijection from the set of vertices of $C_{*}$ onto $\bar{N}$. This implies that $\varphi$ is the identity on the whole chamber $C_{*}$. Now on any other chamber $C_{\gamma}=\gamma C_{*}$ we have by the commutation assumption that $\left.\varphi\right|_{C_{\gamma}}=\left.\gamma \varphi\right|_{C_{*}} \gamma^{-1}$, and thus $\left.\varphi\right|_{C_{\gamma}}=\left.\mathrm{id}\right|_{C_{\gamma}}$. Uniqueness follows.

Since residues are subbuildings, by Lemma 4.14 we obtain the following:

Corollary 4.23 (Product structure of $i^{\perp}$-residues) Fix $i \in I$ and a chamber $C$ of $\Delta$ with center $w$. There exists a type-preserving combinatorial isomorphism $\delta_{i, C}$ from $R(\{i\}, C) \times R\left(i^{\perp}, C\right)$ to $R\left(i^{\perp}, C\right)$ which is equivariant under $\Gamma_{i} \times \Gamma_{i \perp} \rightarrow \Gamma_{i}^{\perp}$ and sends $(w, w)$ to $w$ and both $(p, w)$ and $(w, p)$ to $p$. Such an isomorphism is unique.

\section{Holonomy of a group of type-preserving automorphisms}

Definition 5.1 ( $i$-boundary of residues) Let $R$ denote a residue. The $i$-boundary of $R$, denoted by $\partial_{i} R$, is the union of cubes $Q$ of $R$ such that $i \notin t(Q)$.

Example 5.2 Let $i$ denote some element of $I$. Let us determine the $\{i\}$-residue of $C_{*}$. By Corollary 4.15 , we have $C_{\gamma} \sim_{\{i\}} C_{*} \Longleftrightarrow \gamma \in \Gamma_{i}$. Thus $\bar{R}\left(\{i\}, C_{*}\right)$ is the union of the chambers $\gamma C_{*}$ for $\gamma \in \Gamma_{i}$, and $R\left(\{i\}, C_{*}\right)$ is the union of all edges $\gamma\left[v_{*}, v_{*}^{i}\right]$, for $\gamma \in \Gamma_{i}$, where $v_{*}^{i}$ denotes the unique vertex of $C_{*}$ with type $\{i\}$. So topologically $R\left(\{i\}, C_{*}\right)$ is a cone with vertex $v_{*}^{i}$ The boundary $\partial_{i} R\left(\{i\}, C_{*}\right)$ is just the base of the cone, that is, the set of $q_{i}$ rank-0 vertices contained in $R\left(\{i\}, C_{*}\right)$.

In this example, we observe a general fact: the stabilizer of a residue $R$ in $\operatorname{Aut}_{0}(\Delta)$ acts on the $i$-boundary of $R$.

Lemma 5.3 Let $i, j$ denote distinct adjacent vertices of $\mathcal{G}$. Let $R$ denote the $\{i, j\}-$ residue of some chamber $C$. Then the center of a chamber $C^{\prime}$ of $R$ is in the same connected component of $\partial_{i} R$ as the center of $C$ if and only if $C$ and $C^{\prime}$ are $j$-adjacent. 

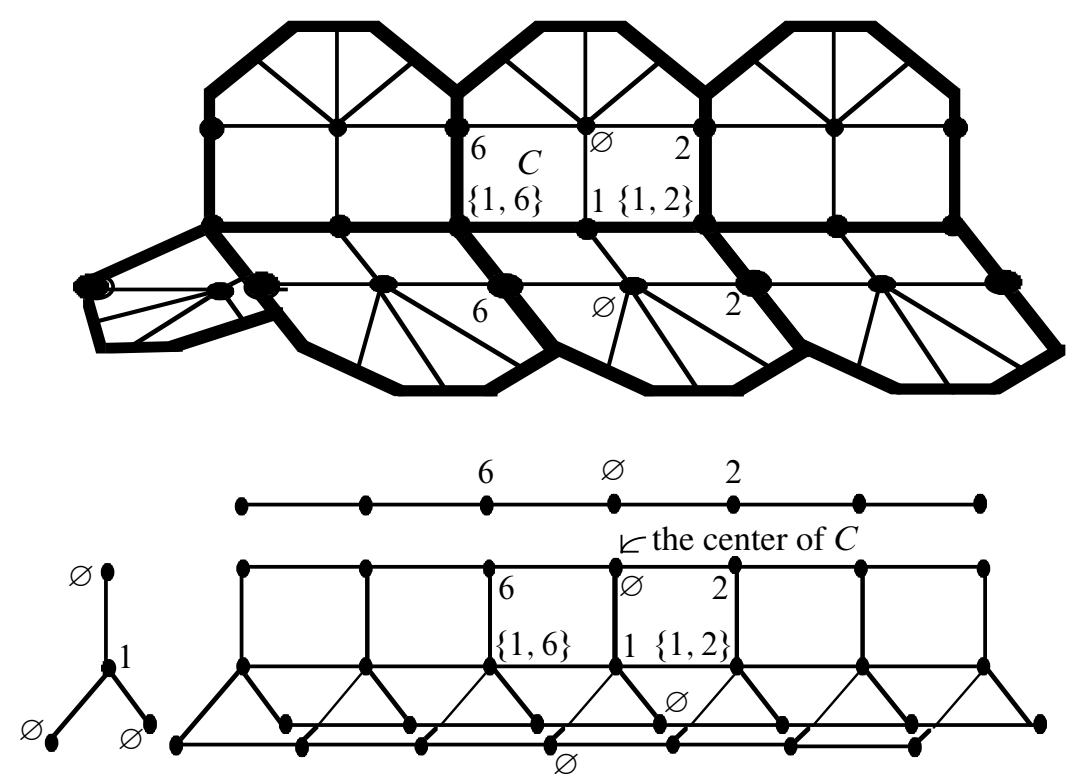

Figure 3: The building is associated to a graph product of groups along a circular graph on $\{1,2,3,4,5,6\}$. The group $G_{1}$ is of order 3 . The groups $G_{2}$ and $G_{6}$ are of order 2 . We have $1^{\perp}=\{2,6\}$ and $1^{\perp}=\{1,2,6\}$. Above we show the extended $\{1,2,6\}$-residue of a chamber $C$. Below we show the corresponding $\{1,2,6\}$-residue, product of the (finite) $\{1\}$-residue and of the $\{2,6\}$-residue, a subdivided line in this case.

Proof The "if" part is obvious. Conversely let $w, w^{\prime}$ denote the centers of $C$ and $C^{\prime}$ and assume that there exists a sequence $\left(v_{0}=w, v_{1}, \cdots, v_{n}=w^{\prime}\right)$ of vertices such that each $\left\{v_{i}, v_{i+1}\right\}$ is contained in an edge of $\partial_{i} R$. Necessarily $t\left(v_{k}\right) \subset\{j\}$. But by Lemma 4.8, each rank-0 vertex is adjacent to a unique vertex of type $\{j\}$ and to no other rank-0 vertex. Thus in the family $\left\{v_{0}, v_{1}, \cdots, v_{n}\right\}$ there is at most one vertex of type $\{j\}$, and all other vertices are of rank 0 . We deduce that $w^{\prime}$ and $w$ are $j$-adjacent.

We may reformulate the lemma above in the following way: the connected components of $\partial_{i} R$ are the $\{j\}$-residues contained in $R$.

Lemma 5.4 Let $C$ be a chamber and $i$ an element of $I$. Then $\delta_{i, C}$ induces a typepreserving $\Gamma_{i} \perp^{\perp}$-equivariant isomorphism $\partial_{i} R(\{i\}, C) \times R\left(i^{\perp}, C\right) \rightarrow \partial_{i} R\left(i^{\perp}, C\right)$. The inclusion $\partial_{i} R(\{i\}, C) \rightarrow \partial_{i} R\left(i^{\perp}, C\right)$ induces a bijective map from $\partial_{i} R(\{i\}, C)$ to $\pi_{0}\left(\partial_{i} R\left(i^{\perp}, C\right)\right)$. Composing this with the identification $\mathcal{C}(R(\{i\}, C)) \rightarrow \partial_{i} R(\{i\}, C)$, we get a bijection $\mathcal{C}(R(\{i\}, C)) \rightarrow \pi_{0}\left(\partial_{i} R\left(i^{\perp}, C\right)\right)$. 
Proof The inclusion $R(\{i\}, C) \subset R\left(i^{\perp}, C\right)$ leads the injective map from $\partial_{i} R(\{i\}, C)$ to $\partial_{i} R\left(i^{\perp}, C\right)$. Remember from Example 5.2 that $\partial_{i} R(\{i\}, C)$ consists of isolated rank-0 vertices, so the injection induces a map $\partial_{i} R(\{i\}, C) \rightarrow \pi_{0}\left(\partial_{i} R\left(i^{\perp}, C\right)\right)$, and we have to check that this map is a bijection.

By Corollary 4.23, we have an isomorphism $\delta_{i, C}: R(\{i\}, C) \times R\left(i^{\perp}, C\right) \rightarrow R\left(i^{\perp}, C\right)$ sending a vertex $\left(v_{1}, v_{2}\right)$ to a vertex of type $t\left(v_{1}\right) * t\left(v_{2}\right)$. In other words $\partial_{i} R\left(i^{\perp}, C\right)$ is the image under $\delta_{i, C}$ of the cubes $\left\{w^{\prime}\right\} \times Q$, where $w^{\prime}$ is a rank-0 vertex of $R(\{i\}, C)$ and $Q$ is any cube of $R\left(i^{\perp}, C\right)$ (recall that $\left.i \notin i^{\perp}\right)$. Thus $\delta_{i, C}$ induces an isomorphism $\partial_{i} R(\{i\}, C) \times R\left(i^{\perp}, C\right) \rightarrow \partial_{i} R\left(i^{\perp}, C\right)$ which is type-preserving.

Residues are connected, hence sending $w^{\prime}$ to $\left(w^{\prime}, w\right)$ identifies $\pi_{0}\left(\partial_{i} R(\{i\}, C)\right)$ with $\pi_{0}\left(\partial_{i} R(\{i\}, C) \times R\left(i^{\perp}, C\right)\right)$, where $w$ denotes the center of the chamber $C$. Composing with $\delta_{i, C}$ yields the desired bijection.

Corollary 5.5 Let $i$ and $j$ denote distinct adjacent vertices of $\mathcal{G}$. Let $R$ denote the $\{i, j\}$-residue of some chamber $C$. Then the center of a chamber $C^{\prime}$ of $R$ is in the same connected component of $\partial_{i} R\left(i^{\perp}, C\right)$ as the center of $C$ if and only if $C$ and $C^{\prime}$ are $j$-adjacent.

Proof It is enough to prove that the inclusion $\partial_{i} R(\{i, j\}, C) \subset \partial_{i} R\left(i^{\perp}, C\right)$ induces a bijection in $\pi_{0}$ by Lemma 5.3.

Now each inclusion $R(\{i\}, C) \rightarrow \pi_{0}\left(\partial_{i} R(\{i, j\}, C)\right)$ and $R(\{i\}, C) \rightarrow \pi_{0}\left(\partial_{i} R\left(i^{\perp}, C\right)\right)$ is bijective by Lemma 5.4. To deduce the first bijectivity remember that $R(\{i, j\}, C)$ is itself a right-angled building, in which $i \stackrel{\perp}{=}=\{i, j\}$.

Let $G$ denote any subgroup of $\operatorname{Aut}_{0}(\Delta)$. For any subcomplex $Y \subset \Delta$ we denote by $G_{Y}$ the stabilizer of $Y$ in $G$, that is, the set of $g \in G$ such that $g Y=Y$.

Definition 5.6 Let $G \subset \operatorname{Aut}_{0}(\Delta)$ denote a subgroup. Let $i$ denote some element of $I$ and let $R$ denote some $i^{\perp}$-residue of $\Delta$. The $i$-holonomy of $G$ at $R$ is the morphism $G_{R} \rightarrow \mathfrak{S}\left(\pi_{0}\left(\partial_{i} R\right)\right)$.

We say that the group $G$ has no holonomy if for each $i \in I$ and each $i^{\perp}$-residue $R$, the $i$-holonomy of $G$ at $R$ is trivial.

Observe that if $G_{i}$ is finite of cardinality $q_{i}$ then $\mathfrak{S}\left(\pi_{0}\left(\partial_{i} R\right)\right)$ is the finite group $\mathfrak{S}\left(q_{i}\right)$. This is because for any chamber $C$ whose center is inside $R$ we know by Lemma 5.4 that we have an identification $\mathcal{C}(R(\{i\}, C)) \rightarrow \pi_{0}\left(\partial_{i} R\right)$, and we have already seen that $\mathcal{C}(R(\{i\}, C))$ admits a simply transitive action of a conjugate of $\Gamma_{i}=G_{i}$. 
Example 5.7 For each graph product $\Gamma=\Gamma\left(\mathcal{G},\left(G_{i}\right)_{i \in I}\right)$ we define $\Gamma_{0}$ as the kernel of the surjective morphism $\Gamma \rightarrow \prod_{i} G_{i}$ inducing the identity on each group $G_{i}$.

If each group $G_{i}$ has cardinality $q_{i}$ then $\Gamma_{0}$ is a subgroup of $\Gamma$ of index $\prod_{i} q_{i}$.

The group $\Gamma_{0}$ has no holonomy. To check this let us first compute the $i$-holonomy of $\Gamma_{0}$ at $C_{*}$. The stabilizer of $R\left(i^{\perp}, C_{*}\right)$ in $\Gamma$ is $\Gamma_{i}^{\perp}$. Thus the stabilizer of $R\left(i^{\perp}, C_{*}\right)$ in $\Gamma_{0}$ is the kernel of $\Gamma_{i} \perp \mapsto \prod_{j \in i} \perp G_{j}$. In particular this stabilizer is contained in $\Gamma_{i \perp}$.

But by Lemma 5.4, it is clear that the action of $\Gamma_{i \perp}$ on $\pi_{0}\left(\partial_{i} R\left(i^{\perp}, C_{*}\right)\right)$ is trivial. Thus the $i$-holonomy of $\Gamma_{0}$ at $C_{*}$ is trivial.

Now $\Gamma_{0}$ is normal in $\Gamma$ and $\Gamma$ is transitive on chambers. It follows that the $i$-holonomy of $\Gamma_{0}$ at any chamber is trivial.

\section{Atlases on a regular right-angled building}

Definition 6.1 An atlas $\mathcal{A}$ on $\Delta$ consists in the following data: for each $i \in I$ and each $i^{\perp}$-residue $R$ we are given a simply transitive action of $G_{i}$ on the set $\pi_{0}\left(\partial_{i} R\right)$ of connected components of the $i$-boundary of $R$. We will denote the corresponding representation by $\mathcal{A}_{i, R}: G_{i} \rightarrow \mathfrak{S}\left(\pi_{0}\left(\partial_{i} R\right)\right)$.

We say that two atlases $\mathcal{A}, \mathcal{A}^{\prime}$ are equivalent (written $\mathcal{A} \sim \mathcal{A}^{\prime}$ ) whenever for each $i, R$, there is an element $g_{i, R} \in G_{i}$ such that $\mathcal{A}_{i, R}^{\prime}(g)=\mathcal{A}_{i, R}\left(g_{i, R} g g_{i, R^{-1}}\right)$ for all $g \in G_{i}$. We denote by $[\mathcal{A}]$ the equivalence class of the atlas $\mathcal{A}$.

Assume $\varphi$ is a type-preserving automorphism of $\Delta$. Then we may set $\mathcal{A}_{i, R}^{\prime}(g)$ equal to $\varphi \circ \mathcal{A}_{i, \varphi^{-1}(R)}(g) \circ \varphi^{-1}$. We thus obtain a new atlas $\mathcal{A}^{\prime}=\varphi_{*}(\mathcal{A})$. This defines an action of $\operatorname{Aut}_{0}(\Delta)$ on the set of all atlases on $\Delta$. Clearly this action preserves equivalence of atlases. The stabilizer of the atlas $\mathcal{A}$ in this action will be denoted by $\operatorname{Aut}_{0}(\Delta, \mathcal{A})$. Similarly we denote by $\operatorname{Aut}_{0}(\Delta,[\mathcal{A}])$ the stabilizer of the equivalence class of an atlas $\mathcal{A}$.

Example 6.2 (The standard atlas on $\Delta\left(\mathcal{G},\left(G_{i}\right)_{i \in I}\right)$ ) We may use the group $\Gamma$ to define atlases on $\Delta$.

(1) More precisely for $i \in I$ and for some $i^{\perp}$-residue $R$, choose any chamber $C$ in $R$. By Corollary 4.15 and Lemma 5.4, the stabilizer $\Gamma_{R(\{i\}, C)}$ is conjugate to $\Gamma_{i}$ in $\Gamma$ and acts simply transitively on $\pi_{0}\left(\partial_{i} R\right)$. Using one of the possible conjugations we get a simply transitive action of $\Gamma_{i}=G_{i}$ on $\pi_{0}\left(\partial_{i} R\right)$. This defines an atlas. Using the 
other possible conjugations yields equivalent atlases, and in fact a whole equivalence class of atlases. The atlases obtained that way we call $\Gamma$-atlases.

Now $\Gamma$ preserves the set of $\Gamma$-atlases. So if $\mathcal{A}$ is a $\Gamma$-atlas then $\Gamma \subset \operatorname{Aut}_{0}(\Delta,[\mathcal{A}])$.

Note that if for all $i$ the group $G_{i}$ is abelian then the equivalence relation is trivial, and there is only one $\Gamma$-atlas. In this case we have $\Gamma \subset \operatorname{Aut}_{0}(\Delta, \mathcal{A})$.

(2) In fact it is always possible to define more carefully a $\Gamma$-atlas $\mathcal{A}$ in such a way that $\Gamma \subset \operatorname{Aut}_{0}(\Delta, \mathcal{A})$.

Consider an element $i \in I$. By Corollary 4.15, the action of $\Gamma_{i}=G_{i}$ on $\mathcal{C}\left(R\left(\{i\}, C_{*}\right)\right)$ is simply transitive. We define $G_{i}{ }^{*}$ as the subgroup of $\mathfrak{S}\left(\mathcal{C}\left(R\left(\{i\}, C_{*}\right)\right)\right)$ consisting in those permutations $\sigma$ which commute with each element $g \in G_{i}$ (acting on $\mathcal{C}\left(R\left(\{i\}, C_{*}\right)\right)$ through $\left.\Gamma_{i}\right)$. So if $\sigma \in G_{i}{ }^{*}$ then for any $g \in G_{i}$ we must have $\sigma\left(g C_{*}\right)=g \sigma\left(C_{*}\right)$. If we define an element $h_{\sigma} \in G_{i}$ by the relation $\sigma\left(C_{*}\right)=h_{\sigma}{ }^{-1} C_{*}$ then we have $\sigma\left(g C_{*}\right)=g h_{\sigma}{ }^{-1} C_{*}$.

Abbreviating the pair $\left(i, R\left(i^{\perp}, C_{*}\right)\right)$ by $\left(i, C_{*}\right)$, let us now define for every $h \in G_{i}$ a permutation $\mathcal{A}_{i, C_{*}}^{\Gamma}(h)$ of $\mathcal{C}\left(R\left(\{i\}, C_{*}\right)\right)$ by the rule $\mathcal{A}_{i, C_{*}}^{\Gamma}(h)\left(g C_{*}\right)=g h^{-1} C_{*}$. We then obtain a map $\mathcal{A}_{i, C_{*}}^{\Gamma}: G_{i} \rightarrow \mathfrak{S}\left(\mathcal{C}\left(R\left(\{i\}, C_{*}\right)\right)\right)$. It is easy to check that $\mathcal{A}_{i, C_{*}}^{\Gamma}$ is an injective morphism with simply transitive image. In fact the image of this morphism is exactly the subgroup $G_{i}{ }^{*}$.

For each $i^{\perp}$-residue $R$ choose an element $\gamma_{R} \in \Gamma$ such that $\gamma_{R} R\left(i^{\perp}, C_{*}\right)=R$. Then define for any $h \in G_{i}$ a permutation of $\pi_{0}\left(\partial_{i} R\right)$ by $\mathcal{A}_{i, R}^{\Gamma}(h)=\gamma_{R} \circ \mathcal{A}_{i, C_{*}}^{\Gamma}(h) \circ \gamma_{R}{ }^{-1}$. Doing this for each $i$ yields an atlas $\mathcal{A}^{\Gamma}$, which we call the standard atlas. Apparently this atlas $\mathcal{A}^{\Gamma}$ depends on the choices of the $\gamma_{R}$, but the following argument shows that it is not the case.

In fact let us show that $\mathcal{A}^{\Gamma}$ is invariant under $\Gamma$. To see this choose $i \in I, R$ a $i^{\perp}$-residue and $\gamma \in \Gamma$. Set $R^{\prime}=\gamma R$. Then we have

$$
\gamma_{*}\left(\mathcal{A}^{\Gamma}\right)_{i, R^{\prime}}(h)=\gamma \circ \mathcal{A}_{i, R}^{\Gamma}(h) \circ \gamma^{-1}=\left(\gamma \gamma_{R}\right) \circ \mathcal{A}_{i, C_{*}}^{\Gamma}(h) \circ\left(\gamma \gamma_{R}\right)^{-1} .
$$

So to prove invariance it suffices to prove independence relative to the choice of the element $\gamma_{R^{\prime}}$.

In fact all this reduces to checking that the action of $\gamma$ on $\pi_{0}\left(\partial_{i} R\left(i^{\perp}, C_{*}\right)\right)$ commutes with the permutation group $\mathcal{A}_{i, C_{*}}^{\Gamma}\left(G_{i}\right)=G_{i}{ }^{*}$ if $\gamma R\left(i^{\stackrel{\perp}{\perp}}, C_{*}\right)=R\left(i^{\perp}, C_{*}\right)$. By Corollary 4.15, the product subgroup $\Gamma_{\{i\}} \times \Gamma_{i \perp}$ is the stabilizer of $R\left(i^{\perp}, C_{*}\right)$ in $\Gamma$. But $\Gamma_{i \perp}$ acts trivially on $\pi_{0}\left(\partial_{i} R\left(i^{\stackrel{\perp}{ }}, C_{*}\right)\right)$, and $G_{i}{ }^{*}$ was defined to commute with $\Gamma_{\{i\}}$. 
Definition 6.3 ( $\mathcal{A}$-word of a gallery) Let $\mathcal{A}$ denote some atlas on $\Delta$. First assume we are given a pair $\left(C, C^{\prime}\right)$ of adjacent chambers: we want to associate to it a letter $s$ in the alphabet $\mathfrak{A}=\{\varnothing\} \sqcup\left(\sqcup_{i}\{i\} \times\left(G_{i} \backslash\{1\}\right)\right)$. If $C^{\prime}=C$ we set $s=\varnothing$. Else by Lemma 4.11, there is one and only one $i$ in $I$ such that $C$ and $C^{\prime}$ are $i$-adjacent. Furthermore using the identification $\mathcal{C}(R(\{i\}, C)) \rightarrow \pi_{0}\left(\partial_{i} R\left(i^{\perp}, C\right)\right)$ of Lemma 5.4 together with the simply transitive representation $\mathcal{A}_{i, R\left(i^{\perp}, C\right)}: G_{i} \rightarrow \pi_{0}\left(\partial_{i} R\left(i^{\perp}, C\right)\right)$, we obtain a simply transitive action of $G_{i}$ on $\mathcal{C}(R(\{i\}, C)$ ) (the corresponding morphism will be still denoted by $\mathcal{A}_{i, R\left(i^{\perp}, C\right)}$ ). So there exists a unique $g_{i} \in G_{i}$ sending $C$ to $C^{\prime}$ (and $g_{i} \neq 1$ because $\left.C^{\prime} \neq C\right)$. We then set $s=\left(i, g_{i}\right)$.

Now to any gallery $\left(C_{0}, C_{1}, \ldots, C_{n}\right)$ we associate the word $\left(s_{1}, \ldots, s_{n}\right)$ in $\mathfrak{A}$ with $s_{i}$ the letter associated as above to the length one gallery $\left(C_{i-1}, C_{i}\right)$.

Lemma 6.4 Let $\left(s_{1}, \ldots, s_{n}\right)$ denote some word in $\mathfrak{A}$. Fix a chamber $C$ and an atlas $\mathcal{A}$. Then there exists a unique gallery $\left(C_{0}, C_{1}, \ldots, C_{n}\right)$ with $C_{0}=C$ and whose associated $\mathcal{A}$-word is $\left(s_{1}, \ldots, s_{n}\right)$.

Proof Using induction on length, it is enough to handle the case $n=1$. If $s_{1}=\varnothing$ then the $\mathcal{A}$-word associated to $\left(C_{0}, C_{1}\right)$ is $s_{1}$ if and only if $C_{1}=C_{0}$. If $s_{1}=\left(i, g_{i}\right)$ then necessarily $C_{1} \in R\left(\{i\}, C_{0}\right)$ and in fact using the simply transitive action of $G_{i}$ on $\mathcal{C}(R(\{i\}, C))$ described in Definition 6.3 we must have $C_{1}=\mathcal{A}_{i, R\left(i \stackrel{\perp}{ }, C_{0}\right)}\left(g_{i}\right) C_{0}$. Conversely the gallery $\left(C_{0}, \mathcal{A}_{i, R\left(i^{\perp}, C_{0}\right)}\left(g_{i}\right) C_{0}\right)$ has the desired property.

Proposition 6.5 Let $\mathcal{A}, \mathcal{A}^{\prime}$ denote two atlases on $\Delta$. Let $C$ and $C^{\prime}$ denote two chambers of $\Delta$, and let $f: C \rightarrow C^{\prime}$ denote the unique type-preserving combinatorial isomorphism between the two chambers. Then $f$ admits one and only one extension $\bar{f} \in \operatorname{Aut}_{0}(\Delta)$ such that $\bar{f}_{*}(\mathcal{A})=\mathcal{A}^{\prime}$.

Proof We first give the proof of uniqueness, which then will lead to existence.

To get uniqueness we show that if a type-preserving extension is already defined on some chamber, then it is uniquely defined on each adjacent chamber. In order to give the argument, we introduce the notion of a germ.

Definition 6.6 (Germs) A germ of $\operatorname{Aut}_{0}(\Delta)$ is a type-preserving combinatorial isomorphism between two chambers.

Remember that by Lemma 4.8 the type $t$ realizes a bijection between the set of vertices of a given chamber and $\bar{N}$. So in this context the pseudo-group of germs is the same as the pseudo-group of pairs of chambers. Observe that the proposition we are proving 
just says that any germ has a unique type-preserving extension sending a given atlas to an other given atlas.

For any gallery $G=\left(C_{0}, C_{1}, \ldots, C_{n}\right)$ with origin $C_{0}=C$ we define a gallery $f(G)$ as follows: $f(G)$ is the unique gallery with origin $C^{\prime}$ and whose $\mathcal{A}^{\prime}$-word is the $\mathcal{A}$-word of $G$ (see Definition 6.3 and Lemma 6.4).

We then define a sequence of germs $G(f)=\left(f_{0}, f_{1}, \ldots, f_{n}\right)$ as follows. Write $f(G)=\left(C_{0}^{\prime}, C_{1}^{\prime}, \ldots, C_{n}^{\prime}\right)$ and define $f_{i}$ as the unique germ sending $C_{i}$ to $C_{i}^{\prime}$.

We claim that we have $f(G)=\bar{f}(G)$ for any type-preserving extension $\bar{f}$ of $f$ and any gallery $G=\left(C_{0}, C_{1}, \ldots, C_{n}\right)$ with origin $C_{0}=C$, and hence the restriction of $\bar{f}$ to $C_{n}$ is the germ $f_{n}$. Uniqueness follows.

To check the claim it is enough to handle the case when $n=1$ and $C_{1} \neq C_{0}$ by induction. Write $s_{1}=\left(i, g_{i}\right)$. By assumption we have $\bar{f}\left(C_{0}\right)=C_{0}^{\prime}$, and hence $\bar{f}\left(R\left(i^{\perp}, C_{0}\right)\right)=R\left(i^{\perp}, C_{0}^{\prime}\right)$. To simplify notation set $R=R\left(i^{\perp}, C_{0}\right), R^{\prime}=R\left(i^{\perp}, C_{0}^{\prime}\right)$. The condition $\bar{f}_{*}(\mathcal{A})=\mathcal{A}^{\prime}$ says that $\mathcal{A}_{i, R^{\prime}}^{\prime}=\bar{f} \circ \mathcal{A}_{i, R} \circ \overline{f^{-1}}$. Now we may determine the chamber $\bar{f}\left(C_{1}\right)$. We have

$$
\bar{f}\left(C_{1}\right)=\bar{f}\left(\mathcal{A}_{i, R}\left(g_{i}\right) C_{0}\right)=\bar{f}\left(\mathcal{A}_{i, R}\left(g_{i}\right)\left(\overline{f^{-1}}\left(C_{0}^{\prime}\right)\right)\right)=\mathcal{A}_{i, R^{\prime}}^{\prime}\left(g_{i}\right) C_{0}^{\prime} .
$$

This latter chamber is $C_{1}^{\prime}$ because the $\mathcal{A}^{\prime}$-word associated to $\left(C_{0}^{\prime}, C_{1}^{\prime}\right)$ is $\left(s_{1}\right)$.

We now prove the existence of a type-preserving extension $\bar{f}$ sending $\mathcal{A}$ to $\mathcal{A}^{\prime}$, still using the action of the germ $f$ on galleries with origin $C$.

For any gallery $G=\left(C_{0}, C_{1}, \ldots, C_{n}\right)$ with $C_{0}=C$ and $C_{n}=D$ we consider the germ $\omega(G(f))=f_{n}$ (from $C_{n}$ to the last chamber $C_{n}^{\prime}$ of $f(G)$ ). We first prove that this germ is independent of the gallery $G$ with endpoints $C, D$.

Observe the product rule $\omega\left(G_{1} G_{2}(f)\right)=\omega\left(G_{2}\left(\omega\left(G_{1}(f)\right)\right)\right)$. If we denote by $\bar{G}$ the inverse gallery of $G$ we also have $\omega(G \bar{G}(f))=f$. This is because the letter associated to the gallery $\left(C_{1}, C_{0}\right)$ is $s^{-1}$, where $s$ is the letter associated to $\left(C_{0}, C_{1}\right)$, and the inverse of a letter $s$ is $\varnothing$ if $s=\varnothing$ and $\left(i, g_{i}{ }^{-1}\right)$ if $s=\left(i, g_{i}\right)$. In other words $\omega(G(f))=\omega\left(G^{\prime}(f)\right)$ whenever $G$ and $G^{\prime}$ are (rank-1)-homotopic (see Definition 4.20).

If $G, G^{\prime}$ are galleries from $C$ to $D$ we have $\omega\left(G \overline{G^{\prime}} G^{\prime}(f)\right)=\omega\left(G^{\prime}\left(\omega\left(G \overline{G^{\prime}}(f)\right)\right)\right)$ and also $\omega\left(G \overline{G^{\prime}} G^{\prime}(f)\right)=\omega\left(\overline{G^{\prime}} G^{\prime}(\omega(G(f)))\right)=\omega(G(f))$. So to conclude that $\omega(G(f))=\omega\left(G^{\prime}(f)\right)$ it is enough to prove that for every closed gallery $G$ with origin $C$ and any germ $f: C \rightarrow \Delta$ we have $\omega(G(f))=f$.

By Lemma 4.21, every closed gallery of $\Delta$ is a product of lassoes, up to rank-1 homotopies. So it is enough to check that if $G=\left(C_{0}=C, C_{1}, C_{2}, C_{3}, C_{4}=C\right)$ then 
$\omega(G(f))=f$ where $C_{0} \sim_{i} C_{1}, C_{0} \neq C_{1}, C_{1} \sim_{j} C_{2}, C_{1} \neq C_{2}, C_{2} \sim_{i} C_{3}, C_{2} \neq C_{3}$, $C_{3} \sim_{j} C_{4}, C_{3} \neq C_{4}$, for distinct adjacent vertices $i$ and $j$ of $\mathcal{G}$.

The $\mathcal{A}$-word of $G$ is then

$$
\left(s_{1}, s_{2}, s_{3}, s_{4}\right)=\left(\left(i, g_{i}\right),\left(j, g_{j}\right),\left(i, g_{i}^{\prime}\right),\left(j, g_{j}^{\prime}\right) .\right.
$$

Note that the four chambers belong to $R\left(i^{\perp}, C\right)$. Since $C_{1}$ and $C_{2}$ are $j$-adjacent they define the same connected component in $\partial_{i} R\left(i^{\perp}, C\right)$ (see Lemma 5.3). The same is true for $C_{0}, C_{3}$. We deduce that $g_{i}^{\prime}=g_{i}{ }^{-1}$. Similarly we have $g_{j}^{\prime}=g_{j}{ }^{-1}$.

Now let us prove that the gallery $f(G)$ is closed: this will conclude because $\omega(G(f))$ will be the unique germ from $C_{4}=C_{0}$ to $C_{4}^{\prime}=C_{0}^{\prime}$, so $\omega(G(f))=f$.

First we note that the centers of $C_{3}^{\prime}$ and $C_{0}^{\prime}$ define the same connected component in $\partial_{i} R\left(i^{\perp}, C_{0}^{\prime}\right)$. Because so do $C_{2}^{\prime}$ and $C_{1}^{\prime}$ (they are $j$-adjacent), and furthermore $g^{\prime}{ }_{i}=g_{i}{ }^{-1}$. This implies that $C_{3}^{\prime}$ and $C_{0}^{\prime}$ are $j$-adjacent by Lemma 5.3. Note that $C_{0}^{\prime} \neq C_{3}^{\prime}$, else $g_{i}^{\prime}=g_{i}{ }^{-1}$ would imply $C_{2}^{\prime}=C_{1}^{\prime}$ thus $g_{j}=1$. So there is an element $h_{j} \in G_{j} \backslash\{1\}$ such that $\mathcal{A}_{j, R\left(j^{\perp}, C_{3}^{\prime}\right)}^{\prime}\left(h_{j}\right) C_{3}^{\prime}=C_{0}^{\prime}$. The same argument as above shows that $h_{j}=g_{j}^{-1}$. So in fact $h_{j}=g_{j}^{\prime}$ and $C_{4}^{\prime}=C_{0}^{\prime}$.

So for any chamber $D$ there is a germ $f_{D}: D \rightarrow \Delta$ such that for any gallery $G$ from $C$ to $D$, we have $\omega(G(f))=f_{D}$. Let us check that all the germs $f_{D}$ fit together and define a type-preserving combinatorial map $\bar{f}$. So let $Q$ denote any cube of $\Delta$, and let $D, D^{\prime}$ denote any two chambers that contain $Q$. We must check that $f_{D}=f_{D^{\prime}}$ on $Q$. Let $v$ denote the vertex of $Q$ with type $J=\underline{t}(Q)$. By Lemma 4.8 the group $\Gamma_{v}$ acts simply transitively on the set $\mathcal{C}(v)$ of chambers containing $v$. Let $\gamma \in \Gamma$ be such that $D^{\prime}=\gamma D$, and let $g \in \Gamma$ be such that $D=g C_{*}$. The conjugate $\gamma^{\prime}=g^{-1} \gamma g$ belongs to $\Gamma_{J}$ by Lemma 4.8. Consider any decomposition $\gamma^{\prime}=g_{1} \ldots g_{n}$ as a product of elements of $G_{i}, i \in J$ : this yields a gallery $\left(C_{*}, g_{1} C_{*}, \ldots, \gamma^{\prime} C_{*}\right)$ from $C_{*}$ to $g^{-1} D^{\prime}$, all of whose chambers contain $g^{-1} Q$. Applying $g$ yields a gallery from $D$ to $D^{\prime}$, all of whose chambers contain $Q$. Thus we see that it suffices to argue in the case when $D, D^{\prime}$ are $i$-adjacent for some $i \in J$. Let $w$ denote the unique vertex in $D \cap D^{\prime}$ of type $\{i \underline{Q}$. There is a unique smallest cube $\bar{Q}$ in $D$ containing $Q$ and $w$. By Lemma 4.18, $\bar{Q}$ is also in $D^{\prime}$. We note that the image chambers $E$ and $E^{\prime}$ of the germs $f_{D}$ and $f_{D^{\prime}}$ respectively are $i$-adjacent, because $f_{D^{\prime}}$ is obtained by extending $f_{D}$ along the gallery $\left(D, D^{\prime}\right)$. Since $E \cap E^{\prime}$ contains a unique vertex of type $\{i\}$, the germs $f_{D}$ and $f_{D^{\prime}}$ are equal on $w$. In fact by Lemma 4.18 , the germs $f_{D}$ and $f_{D^{\prime}}$ are equal on $\bar{Q}$, hence on $Q$.

Now assume $\mathcal{A}^{\prime \prime}$ is a second atlas, $C^{\prime \prime}$ is a second chamber and $f^{\prime}$ denotes the germ sending $C^{\prime}$ to $C^{\prime \prime}$. Then it is straightforward that $\left(f^{\prime} f\right)(G)=f^{\prime}(f(G))$. This implies 
that the $i$-th germ of $G\left(f^{\prime} f\right)$ is $G\left(f^{\prime} f\right)_{i}=(f(G))\left(f^{\prime}\right)_{i} \circ G(f)_{i}$. So $\overline{f^{\prime} f}=\overline{f^{\prime}} \bar{f}$. In particular we have $\overline{f^{-1}} \bar{f}=\mathrm{id}_{\Delta}$. So $\bar{f}$ is in fact a type-preserving automorphism extending $f$.

It remains to check that $\bar{f}_{*}\left(\mathcal{A}^{\prime}\right)=\mathcal{A}$. So let $i \in I$ and let $R$ denote some $i^{\perp}$-residue. Fix a chamber $D$ in $\mathcal{C}(R)$ and a gallery $G=\left(C_{0}=C, \ldots, C_{n}=D\right)$. Set $R^{\prime}=\bar{f}(R)$. We must show that for every $g \in G_{i}$ we have $\mathcal{A}_{i, R^{\prime}}^{\prime}(g) \circ \bar{f}=\bar{f} \circ \mathcal{A}_{i, R}(g)$ on $\pi_{0}\left(\partial_{i} R\right)$, or equivalently on $\mathcal{C}(R(\{i\}, D))$ using the identification $\mathcal{C}(R(\{i\}, D)) \rightarrow \pi_{0}\left(\partial_{i} R\right)$ of Lemma 5.4. Since the relation is obvious if $g=1$ we will assume $g \neq 1$.

Set $E=\mathcal{A}_{i, R}(g) D$. Consider the gallery $H=G \cdot(D, E)=\left(C_{0}, \ldots, C_{n}, E\right)$. Let $H^{\prime}=\left(C_{0}^{\prime}, \ldots, C_{n}^{\prime}, E^{\prime}\right)$ denote its image under $\bar{f}$. By construction the last letter in the $\mathcal{A}^{\prime}$-word of $H^{\prime}$ is $(i, g)$. By definition it means that $E^{\prime}$ is $i$-adjacent to $D^{\prime}$ with $E^{\prime}=\mathcal{A}_{i, R^{\prime}}^{\prime}(g) D^{\prime}$. So we have checked the relations $\mathcal{A}_{i, R^{\prime}}^{\prime}(g)(\bar{f}(D))=\bar{f}\left(\mathcal{A}_{i, R}(g) D\right)$ for every $g \in G_{i}$. This was done without assumption on $D$.

Corollary 6.7 If $\mathcal{A}$ is an atlas on $\Delta$ then the automorphism group $\operatorname{Aut}_{0}(\Delta, \mathcal{A})$ acts simply transitively on chambers. For example $\operatorname{Aut}_{0}\left(\Delta, \mathcal{A}^{\Gamma}\right)=\Gamma$. In general $\operatorname{Aut}_{0}(\Delta, \mathcal{A})$ is conjugate to $\Gamma$ inside $\operatorname{Aut}_{0}(\Delta)$.

Proof The first assertion follows from Proposition 6.5 applied with $\mathcal{A}^{\prime}=\mathcal{A}$; for the case of $\mathcal{A}^{\Gamma}$ see Example 6.2. The second assertion then follows from Proposition 6.5 applied with $\mathcal{A}^{\prime}=\mathcal{A}^{\Gamma}$.

Proposition 6.8 Let $\Gamma^{\prime}$ denote a subgroup of $\operatorname{Aut}_{0}(\Delta)$ with no holonomy. Then there exists an atlas $\mathcal{A}$ on $\Delta$ such that $\Gamma \subset \operatorname{Aut}_{0}(\Delta, \mathcal{A})$.

Proof Let $i \in I$ denote a fixed element. Choose a family $\left(R_{t}\right)_{t \in T}$ of $i^{\perp}$-residues such that every $i^{\perp}$-residue is in the $\Gamma^{\prime}$-orbit of $R_{t}$ for one and only one $t$. Choose arbitrarily a simply transitive representation $\mathcal{A}_{i, R_{t}}: G_{i} \rightarrow \mathfrak{S}\left(\pi_{0}\left(\partial_{i} R_{t}\right)\right)$ for each residue $R_{t}$.

Now for any $i^{\perp}$-residue $R$ there is some $\gamma^{\prime} \in \Gamma^{\prime}$ and a unique $t \in T$ and such that $R=\gamma^{\prime} R_{t}$. The simply transitive representation $\mathcal{A}_{i, R}: G_{i} \rightarrow \mathfrak{S}\left(\pi_{0}\left(\partial_{i} R\right)\right)$ defined by $\mathcal{A}_{i, R}(g)=\gamma^{\prime} \circ \mathcal{A}_{i, R_{t}} \circ \gamma^{\prime-1}$ is in fact independent of the choice of $\gamma^{\prime}$. Indeed for another $\gamma^{\prime \prime} \in \Gamma^{\prime}$ sending $R_{t}$ to $R$ we have

$$
\gamma^{\prime} \circ \mathcal{A}_{i, R_{t}} \circ \gamma^{\prime-1}=\left(\gamma^{\prime} \gamma^{\prime \prime-1}\right) \circ \gamma^{\prime \prime} \circ \mathcal{A}_{i, R_{t}} \circ \gamma^{\prime \prime-1} \circ\left(\gamma^{\prime} \gamma^{\prime \prime-1}\right)^{-1} \text {. }
$$

But $\Gamma^{\prime}$ has no holonomy, so the element $\gamma^{\prime} \gamma^{\prime \prime}-1 \in \Gamma_{R}^{\prime}$ acts trivially on $\pi_{0}\left(\partial_{i} R\right)$, and we are done. 
If we do this for each $i \in I$ we get an atlas $\mathcal{A}$ on $\Delta$. We now check that this atlas is invariant under $\Gamma^{\prime}$.

Let $i \in I$ be a vertex of $\mathcal{G}$, let $R$ denote some $i^{\perp}$-residue and let $\varphi$ belong to $\Gamma^{\prime}$. Set $R^{\prime}=\varphi(R)$. For $g \in G_{i}$ we have

$$
\varphi \circ \mathcal{A}_{i, R}(g) \circ \varphi^{-1}=\left(\varphi \circ \gamma^{\prime}\right) \circ \mathcal{A}_{i, R_{t}}(g) \circ\left(\varphi \circ \gamma^{\prime}\right)^{-1}
$$

with $\gamma^{\prime} \in \Gamma^{\prime}$ such that $\gamma^{\prime} R_{t}=R$. The element $\varphi \circ \gamma^{\prime} \in \Gamma^{\prime}$ sends $R_{t}$ to $R^{\prime}$, so by the independence property above we have

$$
\left(\varphi \circ \gamma^{\prime}\right) \circ \mathcal{A}_{i, R_{t}}(g) \circ\left(\varphi \circ \gamma^{\prime}\right)^{-1}=\mathcal{A}_{i, R^{\prime}}(g) .
$$

Theorem 6.9 Let $\Gamma^{\prime}$ denote a subgroup of $\operatorname{Aut}_{0}(\Delta)$ with a finite number of $\Gamma^{\prime}$-orbits on the set of chambers of $\Delta$. Assume that $\Gamma^{\prime}$ has a finite index subgroup with no holonomy. Then $\Gamma^{\prime}$ is commensurable to $\Gamma$ in $\operatorname{Aut}_{0}(\Delta)$.

Proof We may assume that $\Gamma^{\prime}$ itself has no holonomy. By Proposition 6.8, there exists an atlas $\mathcal{A}$ on $\Delta$ which is invariant under $\Gamma^{\prime}$. By Corollary $6.7, \operatorname{Aut}_{0}(\Delta, \mathcal{A})$ is simply transitive on chambers. The index of $\Gamma^{\prime}$ in $\operatorname{Aut}_{0}(\Delta, \mathcal{A})$ is thus precisely the number of $\Gamma^{\prime}$-orbits in the set of chambers of $\Delta$. Hence by assumption, $\Gamma^{\prime}$ is of finite index in $\operatorname{Aut}_{0}(\Delta, \mathcal{A})$.

Again by Corollary 6.7, the groups $\operatorname{Aut}_{0}(\Delta, \mathcal{A})$ and $\Gamma$ are conjugate in $\operatorname{Aut}_{0}(\Delta)$.

Proof of Theorem 1.4 Let $\Gamma^{\prime}$ denote a uniform lattice of $\operatorname{Aut}_{0}(\Delta)$. If $\Gamma^{\prime}$ has a finite index subgroup with no holonomy then by Theorem 6.9, it is commensurable to the graph product $\Gamma$ in $\operatorname{Aut}_{0}(\Delta)$.

Conversely if $\Gamma^{\prime}$ is commensurable with $\Gamma$, consider $f \in \operatorname{Aut}_{0}(\Delta)$ where $\Gamma_{1} \subset \Gamma$ and $\Gamma_{1}^{\prime} \subset \Gamma^{\prime}$ are such that $f \Gamma_{1} f^{-1}=\Gamma_{1}^{\prime}$. Then $\Gamma_{0} \cap \Gamma_{1}$ is a finite index subgroup of $\Gamma$ without holonomy, thus $f\left(\Gamma_{0} \cap \Gamma_{1}\right) f^{-1}$ is a finite index subgroup of $\Gamma^{\prime}$ without holonomy.

Proof of Corollary 1.6 (Leighton's Lemma for biregular graphs) Let $X_{1}$ and $X_{2}$ denote two biregular graphs of valencies $p$ and $q$, respectively. Then $\pi_{1} X_{k}$ are uniform lattices of the biregular tree $T$ of valencies $p$ and $q$. In fact the first barycentric subdivision of $T$ is the right-angled building associated to the free product of $G_{i}=$ $\mathbb{Z} / p \mathbb{Z}$ and $G_{j}=\mathbb{Z} / q \mathbb{Z}$. Observe that the stabilizer of a $i^{\perp}-$ or a $j^{\perp}$-residue in $\pi_{1} X_{k}$ is trivial, because in this case $i^{\perp}=\{i\}$ and $j^{\perp}=\{j\}$. In particular $\pi_{1} X_{k}$ has no holonomy and we may apply Theorem 6.9: either of the fundamental groups are commensurable with the free product $\Gamma=\mathbb{Z} / p \mathbb{Z} * \mathbb{Z} / q \mathbb{Z}$. 
Proof of Corollary 1.5 Fix a graph $\mathcal{G}$ with vertex set $T$. Choose two families of groups $\left(G_{i}\right)_{i \in I},\left(G_{i}^{\prime}\right)_{i \in I}$, such that $\left|G_{i}\right|=\left|G_{i}^{\prime}\right|=q_{i}$ for each $i \in I$. Consider the graph-product $\Gamma$ of $\left\{G_{i}\right\}_{i \in I}$ along $\mathcal{G}$, and set similarly $\Gamma^{\prime}=\Gamma\left(\mathcal{G},\left(G_{i}^{\prime}\right)_{i \in I}\right)$. Let $\Delta$ and $\Delta^{\prime}$ denote the right-angled building associated to the graph products $\Gamma$ and $\Gamma^{\prime}$.

Then $\Delta$ and $\Delta^{\prime}$ are both "regular with parameters $\left(q_{i}\right)_{i \in I}$ ", in the sense that each vertex of type $\{i\}$ is contained in $q_{i}$ chambers. Thus by Proposition 5.1 of Haglund and Paulin [24], the buildings $\Delta$ and $\Delta^{\prime}$ are isomorphic.

This provides an embedding of $\Gamma^{\prime}$ inside $\operatorname{Aut}_{0}(\Delta)$. Now $\Gamma^{\prime}$ is virtually without holonomy by Example 5.7, thus by Theorem 6.9, the group $\Gamma^{\prime} \subset \operatorname{Aut}_{0}(\Delta)$ is commensurable with $\Gamma$.

Remark 6.10 In the proof above we have in fact $\Gamma_{0} \simeq \Gamma_{0}^{\prime}$ because one can check directly that the cube complexes $\Gamma_{0} \backslash \Delta$ and $\Gamma_{0}^{\prime} \backslash \Delta$ are isomorphic.

\section{Killing the holonomy}

In this section we assume that $\mathcal{G}$ is finite and all groups $G_{i}$ are finite, so that $\Delta$ is locally compact (see Corollary 4.9). Let $q_{i}$ denote the cardinality of $G_{i}$.

Definition 7.1 Let $G$ denote any group and let $H$ denote some subgroup of $G$. Then $H$ is said to be separable in $G$ whenever the intersection of finite index subgroups of $G$ containing $H$ reduces to $H$.

Theorem 7.2 Let $\Gamma^{\prime}$ denote some subgroup of $\operatorname{Aut}_{0}(\Delta)$, such that there are only finitely many $\Gamma^{\prime}$-orbits in the set of $i^{\perp}$-residues for each $i \in I$. Assume that for each $i^{\perp}$-residue $R$ any finite index subgroup of $\Gamma_{R}^{\prime}$ is separable in $\Gamma^{\prime}$. Then $\Gamma^{\prime}$ has a finite index subgroup with no holonomy.

Proof Suppose we have fixed $i \in I$ and chosen some $i^{\perp}$-residue $R$. Consider the following two properties:

$\left(P_{1}\right)$ There exists a finite index subgroup $\Gamma^{\prime \prime} \subset \Gamma^{\prime}$ such that the $i$-holonomy of $\Gamma^{\prime \prime}$ at $R$ is trivial.

$\left(P_{2}\right)$ There exists a finite index subgroup $\Gamma^{\prime \prime} \subset \Gamma^{\prime}$ such that for every $\gamma^{\prime} \in \Gamma^{\prime}$ the $i$-holonomy of $\Gamma^{\prime \prime}$ at $\gamma^{\prime} R$ is trivial. 
We first show $\left(P_{1}\right) \Rightarrow\left(P_{2}\right)$. Consider first a finite index subgroup $\Gamma^{\prime \prime}$ such that the $i$-holonomy of $\Gamma^{\prime \prime}$ at $R$ is trivial. Up to replacing $\Gamma^{\prime \prime}$ by the intersection of its conjugates in $\Gamma^{\prime}$ we may assume that $\Gamma^{\prime \prime}$ is normal. Then its $i$-holonomy at $\gamma^{\prime} R$ is trivial for any $\gamma^{\prime} \in \Gamma^{\prime}$.

This is because by the normality assumption $\gamma^{\prime}$ conjugates $\Gamma_{R}^{\prime \prime}$ onto $\Gamma_{\gamma^{\prime} R}^{\prime \prime}$, and equivariantly conjugates the $i$-holonomy representations at $R$ and $\gamma^{\prime} R$.

Now property $\left(P_{2}\right)$ for each pair $(i, R)$ implies the theorem. Indeed choose a finite set $R_{1}, \ldots, R_{n}$ of representative of $i^{\perp}$-residues, $i \in I$, modulo $\Gamma^{\prime}$. Applying $\left(P_{2}\right)$ we find for each $1 \leq j \leq n$ a finite index subgroup $\Gamma^{\prime \prime}{ }_{j}$ such that the $i_{j}$-holonomy of $\Gamma^{\prime \prime}{ }_{j}$ at any translate $\gamma^{\prime} R_{j}$ is trivial. Then the finite intersection $\bigcap_{j} \Gamma^{\prime \prime}{ }_{j}$ is a finite index subgroup with no holonomy.

So it remains to prove $\left(P_{1}\right)$. Let $K_{R}$ denote the kernel of the $i$-holonomy representation $\Gamma^{\prime}{ }_{R} \rightarrow \mathfrak{S}\left(\pi_{0}\left(\partial_{i} R\right)\right)$. Note that $K_{R}$ is a finite index subgroup of $\Gamma^{\prime}{ }_{R}$ because $G_{i}$ is finite. Thus there is a family $\left(\Gamma^{\prime}{ }_{s}\right)_{s \in S}$ of finite index subgroups of $\Gamma^{\prime}$ whose intersection is $K_{R}$. Let $h_{0}=1, h_{1}, \ldots, h_{n}$ denote a set representatives of $\Gamma^{\prime}{ }_{R}$ modulo $K_{R}$. Then there exists $s_{1}, \ldots, s_{n}$ such that $h_{j} \notin \Gamma^{\prime} s_{j}$.

Set $\Gamma^{\prime \prime}=\cap_{j} \Gamma^{\prime} s_{j}$. This a finite index subgroup because the intersection is finite. Furthermore $K_{R} \subset \Gamma^{\prime \prime}$. We claim that in fact $\Gamma^{\prime}{ }_{R} \cap \Gamma^{\prime \prime}=K_{R}$. For $h \in \Gamma^{\prime}{ }_{R} \backslash K_{R}$ we have $h=k s_{j}$ for some $1 \leq j \leq n$ and some $k \in K_{R}$. We cannot have $h \in \Gamma^{\prime \prime}$, else as $k \in \Gamma^{\prime \prime}$ we would have $s_{j} \in \Gamma^{\prime \prime}$, absurd.

Now the $i$-holonomy of $\Gamma^{\prime \prime}$ at $R$ is trivial because $\Gamma^{\prime \prime}{ }_{R}=K_{R}$.

Proof of Theorem 1.3 By Proposition 4.19, residues are convex subcomplexes, thus their stabilizers are quasiconvex subgroups, as well as their finite index subgroups. So Theorem 7.2 applies, and to finish we apply Theorem 6.9.

\section{Part II Virtual triviality of some extensions by finite groups}

\section{Polygonal complexes}

Definition 8.1 (Polygonal complexes) Consider a discrete set $V$. Choose a family of maps $\left\{e_{\alpha}:\{0,1\} \rightarrow V\right\}_{\alpha \in A}$ and use these maps to glue copies of [0,1] along its boundary (one copy for each $e_{\alpha}$ ). This produces a graph $\mathcal{G}$. The maps $e_{\alpha}$ naturally extend to maps $e_{\alpha}:[0,1] \rightarrow \mathcal{G}$, called the basic oriented edges of $\mathcal{G}$. A map $f: \mathcal{G} \rightarrow \mathcal{G}^{\prime}$ between two graphs is combinatorial whenever for each $\alpha$ there is an $\alpha^{\prime}$ such that $f \circ e_{\alpha}=e^{\prime} \alpha^{\prime} \circ i$ where $i$ is an isometry of $[0,1]$. 
Note that each simple polygonal line in Euclidean space has a natural structure of graph for which the basic oriented edges are (restrictions of) affine maps.

Now consider a family $\left\{\pi_{\beta}\right\}_{\beta \in B}$ of convex polygons of $\mathbb{E}^{2}$, together with a family of combinatorial maps $f_{\beta}: \partial \pi_{\beta} \rightarrow \mathcal{G}$. We may glue the polygons $\pi_{\beta}$ to the graph $\mathcal{G}$ along their boundaries using the maps $f_{\beta}$. The resulting space $X$ is a polygonal complex. Then each map $f_{\beta}: \partial \pi_{\beta} \rightarrow \mathcal{G}$ naturally extends to a map $f_{\beta}: \pi_{\beta} \rightarrow X$ (the basic oriented polygons of $X$ ).

The set $V$ is the set of vertices (of $\mathcal{G}$ and of $X$ ). The graph $\mathcal{G}$ is the 1 -skeleton of $X$. A map $f: X \rightarrow X^{\prime}$ is combinatorial if $f(\mathcal{G}) \subset \mathcal{G}^{\prime}$, the restriction $\left.f\right|_{\mathcal{G}}$ is a combinatorial map of graphs and for each oriented polygon $f_{\beta}: \pi_{\beta} \rightarrow X$ we have $f \circ f_{\beta}=f^{\prime}{ }^{\prime} \circ \varphi$, where $\varphi: \pi_{\beta} \rightarrow \pi^{\prime} \beta^{\prime}$ is the restriction of an affine isomorphism. We will denote by $\operatorname{Aut}(X)$ the group of bijective combinatorial maps $X \rightarrow X$.

An oriented edge of $X$ is a combinatorial map $\vec{a}:[0,1] \rightarrow X$. The associated edge $a$ is the image of $\vec{a}$. The initial point of an oriented edge $\vec{a}$ is $\vec{a}(0)$, denoted by $i(\vec{a})$; its terminal point is $\vec{a}(1)$, denoted by $t(\vec{a})$. The inverse of an oriented edge $\vec{a}:[0,1] \rightarrow X$ is the map $\overleftarrow{a}:[0,1] \rightarrow X$ defined by $\overleftarrow{a}(u)=\vec{a}(1-u)$. An oriented polygon of $X$ is a combinatorial map $f: \pi \rightarrow X$ where $\pi$ denotes some Euclidean convex polygon. The associated polygon is the image of $f$. Note that the edges and polygons of $X$ are the images of the basic oriented edges and polygons. For each polygon $\pi$ of $X$ parametrized by the basic oriented polygon $f_{\beta}: \pi_{\beta} \rightarrow X$ we denote by $k_{\pi}$ the number of edges in $\partial \pi_{\beta}$.

We say that a polygonal complex is regular if for each $\beta \in B$ there is an affine isomorphism sending the polygon $\pi_{\beta}$ to a regular Euclidean polygon.

Definition 8.2 (Paths and cycles) A (combinatorial) path of a polygonal complex $X$ is a sequence $c=\left(\overrightarrow{a_{1}}, \ldots, \overrightarrow{a_{n}}\right)$ of oriented edges such that for each integer $0 \leq i<n$ we have $t\left(\overrightarrow{a_{i}}\right)=i\left(\overrightarrow{a_{i+1}}\right)$. A cycle of $X$ is a path $\left(\overrightarrow{a_{1}}, \ldots, \overrightarrow{a_{n}}\right), n>0$, such that $t\left(\overrightarrow{a_{n}}\right)=i\left(\overrightarrow{a_{1}}\right)$ and $\overleftarrow{a_{i}} \neq \overrightarrow{a_{i+1}}$.

Definition 8.3 (Barycentric subdivision) Let $X$ denote a polygonal complex. For any oriented edge $\vec{a}$ of $X$ the center of $\vec{a}$ is the point $\vec{a}(1 / 2)$. For any oriented polygon $f_{\beta}: \pi_{\beta} \rightarrow X$ the center of $f_{\beta}$ is the image under $f_{\beta}$ of the barycenter of $\pi_{\beta}$. Note that these points depend only on the images of the oriented edges or polygons.

We set $V_{0}=V$, the set of vertices of $X$. We denote by $V_{1}$ the set of centers of oriented edges and by $V_{2}$ the set of centers of oriented polygons. We denote by $E_{01}$ the set of restrictions of basic oriented edges of $X$ to $[0,1 / 2]$ or $[1 / 2,1]$. We denote by $F$ the set of restrictions of basic oriented polygons $f_{\beta}$ to triangle with vertices $v, m$, $p$, with 
$p$ the barycenter of $\pi_{\beta}, m$ the barycenter of an edge $e$ of $\pi_{\beta}$ and $v$ a vertex of the edge $e$ (these maps will be called basic triangles). With the same notation we denote by $E_{02}$ the set of restrictions of $f_{\beta}$ to segments of the form [vp], and we denote by $E_{12}$ the set of restrictions of $f_{\beta}$ to segments of the form $[\mathrm{mp}]$.

The barycentric subdivision of $X$ is the multisimplicial complex with set of vertices $V_{0} \sqcup V_{1} \sqcup V_{2}$, set of edges $E_{01} \sqcup E_{02} \sqcup E_{12}$ and set of (basic) triangles $F$. This multisimplicial complex will be denoted by $X^{\prime}$. It is naturally homeomorphic to $X$.

Definition 8.4 (Rank of vertices) For each vertex $v$ of the barycentric subdivision $X^{\prime}$ of a polygonal complex $X$ we set $\operatorname{rk}(v)=i \Longleftrightarrow v \in V_{i}$ for $i \in\{0,1,2\}$. This integer we call the rank of $v$. For every vertex $p$ of rank 2 we will set $k_{p}=k_{\pi}$, where $\pi$ is the polygon whose center is $p$.

We will denote by $\operatorname{Aut}_{\mathrm{rk}}\left(X^{\prime}\right)$ the group of automorphisms of $X^{\prime}$ which preserve the rank.

Remark 8.5 Let $X$ denote a regular polygonal complex. Then the natural inclusion $\operatorname{Aut}(X) \rightarrow \operatorname{Aut}_{\mathrm{rk}}\left(X^{\prime}\right)$ is an isomorphism.

Definition 8.6 (Links) Let $X$ denote a polygonal complex, and let $v$ denote a vertex of $X$. Then $\operatorname{link}\left(v, X^{\prime}\right)$ is the barycentric subdivision of a unique graph, denoted by $\operatorname{link}(v, X)$. Vertices of $\operatorname{link}(v, X)$ correspond to oriented edges of $X$ with initial point $v$, and edges of $\operatorname{link}(v, X)$ correspond to polygons of $X$ containing $v$. For each edge $e$ of $\operatorname{link}(v, X)$ we will set $k_{e}=k_{\pi}$, where $\pi$ is the polygon of $X$ containing $v$ and corresponding to $e$.

Definition 8.7 (Nonpositive curvature conditions) We consider four kinds of nonpositive curvature conditions on a polygonal complex $X$ with vertex set $V$.

(Q) For all $v \in V$, if $c=\left(\overrightarrow{e_{1}}, \ldots, \overrightarrow{e_{n}}\right)$ is a cycle of $\operatorname{link}(v, X)$ then $n \geq 4$, and if $\pi$ is a polygon then $k_{\pi} \geq 4$.

$\left(\mathrm{C}^{4}\right)$ For all $v \in V$, if $c=\left(\overrightarrow{e_{1}}, \ldots, \overrightarrow{e_{n}}\right)$ is a cycle of $\operatorname{link}(v, X)$ then $\sum_{i}\left(\frac{1}{2}-\frac{1}{4 f_{e_{i}}}\right) \geq 1$ where $f_{e_{i}}$ is the integer such that $k_{e_{i}}-4 f_{e_{i}} \in\{0,1,2,3\}$.

$\left(\mathrm{C}^{2}\right)$ For all $v \in V$, if $c=\left(\overrightarrow{e_{1}}, \ldots, \overrightarrow{e_{n}}\right)$ is a cycle of $\operatorname{link}(v, X)$ then $\sum_{i}\left(\frac{1}{2}-\frac{1}{2 t_{e_{i}}}\right) \geq 1$ where $t_{e_{i}}$ is the integer such that $k_{e_{i}}-2 t_{e_{i}} \in\{0,1\}$.

(C) For all $v \in V$, if $c=\left(\overrightarrow{e_{1}}, \ldots, \overrightarrow{e_{n}}\right)$ is a cycle of $\operatorname{link}(v, X)$ then $\sum_{i}\left(\frac{1}{2}-\frac{1}{k_{e_{i}}}\right) \geq 1$.

There are also analogous conditions $\left(\mathrm{Q}^{\prime}\right),\left(\mathrm{C}^{\prime}\right),\left(\mathrm{C}^{2^{\prime}}\right),\left(\mathrm{C}^{4^{\prime}}\right)$ obtained by replacing $n \geq 4$ or $\sum \geq 1$ in $(\mathrm{Q}),(\mathrm{C}),\left(\mathrm{C}^{2}\right),\left(\mathrm{C}^{4}\right)$ by $n>4$ or $\sum>1$.

Clearly $(\mathrm{Q}) \Rightarrow\left(\mathrm{C}^{4}\right) \Rightarrow\left(\mathrm{C}^{2}\right) \Rightarrow(\mathrm{C})$, and $\left(\mathrm{Q}^{\prime}\right) \Rightarrow\left(\mathrm{C}^{4^{\prime}}\right) \Rightarrow\left(\mathrm{C}^{2^{\prime}}\right) \Rightarrow\left(\mathrm{C}^{\prime}\right)$. 
Definition 8.8 (Piecewise Euclidean metrics) We define four types of piecewise Euclidean metrics on (the barycentric subdivision of) our polygonal complexes.

Let $X$ denote a polygonal complex. For each basic triangle $f: T \rightarrow X^{\prime}$ of the barycentric subdivision let $v, m$ and $p$ denote the vertices of $f(T)$ with ranks 0,1 and 2. There exist a unique affine automorphism $\varphi: \mathbb{E}^{2} \rightarrow \mathbb{E}^{2}$ such that the triangle $T_{0}=\varphi^{-1}(T)$ has the following characteristics: the angle of $T_{0}$ at $p_{0}=\varphi^{-1}(p)$ is $\pi / k_{p}$, the angle of $T_{0}$ at $m_{0}=\varphi^{-1}(m)$ is $\pi / 2$ the length of the edge $\left[v_{0} m_{0}\right]$ is $1 / 2$ where $v_{0}=\varphi^{-1}(v)$. We then consider the new triangles $f \circ \varphi$ and observe that two such triangles differ on an edge of $X^{\prime}$ by an isometry. Thus we have defined a structure of $M_{0}$-polyhedral complex on $X^{\prime}$ in the sense of [8, Definition 7.37]. We will call the associated length metric on $X$ the $(\mathrm{C})$-metric.

If no polygon of $X$ is a triangle, we may similarly define on $X$ a $\left(\mathrm{C}^{4}\right)$-metric, a $\left(\mathrm{C}^{2}\right)$ metric and $(\mathrm{Q})$-metric. For the $\left(\mathrm{C}^{4}\right)$-metric, we demand that the angle of the Euclidean triangle $T_{0}$ at $p_{0}$ be $\pi /\left(4 f_{p}\right)$ with $f_{p}$ the integer defined by $k_{p}-4 f_{p} \in\{0,1,2,3\}$. For the $\left(\mathrm{C}^{2}\right)$-metric, we demand that the angle of the Euclidean triangle $T_{0}$ at $p_{0}$ be $\pi /\left(2 t_{p}\right)$ with $t_{p}$ the integer defined by $k_{p}-2 t_{p} \in\{0,1\}$. For the (Q)-metric, we demand that the angle of the Euclidean triangle $T_{0}$ at $p_{0}$ be $\pi / 4$.

Lemma 8.9 If a locally compact polygonal complex $X$ satisfies condition (C) then the (C)-metric on $X$ is locally $\mathrm{CAT}(0)$. The same is true if we replace (C) by $\left(\mathrm{C}^{4}\right)$, $\left(\mathrm{C}^{2}\right)$ or $(\mathrm{Q})$.

If a compact polygonal complex $X$ satisfies condition $\left(\mathrm{Q}^{\prime}\right),\left(\mathrm{C}^{\prime}\right),\left(\mathrm{C}^{2^{\prime}}\right)$ or $\left(\mathrm{C}^{4^{\prime}}\right)$ then $\pi_{1}(X)$ is Gromov-hyperbolic.

Proof In each case $X$ has (locally) finitely many isomorphisms type of polygons and the systole of the metric link of vertices is at least $2 \pi$ (computations are left to the reader). Thus the associated length metric is locally $\mathrm{CAT}(0)$ by [8, Theorem 5.2 and Lemma 5.6, pages 206-207].

Under conditions $\left(\mathrm{Q}^{\prime}\right),\left(\mathrm{C}^{\prime}\right),\left(\mathrm{C}^{2^{\prime}}\right)$ or $\left(\mathrm{C}^{4^{\prime}}\right)$, the systole of the metric link of rank-0 vertices is greater than $2 \pi$. So we may replace our Euclidean triangles by small enough hyperbolic triangles with the same angles at rank-1 and rank-2 vertices. This defines a structure of piecewise hyperbolic simplicial complex in which the link condition is still fulfilled. Thus the associated length metric is locally CAT $(-1)$ [8], and so $\pi_{1}(X)$ is Gromov-hyperbolic by Proposition 1.2 of [8].

Definition 8.10 Let $X$ denote a polygonal complex. Let $\Pi=\Pi(X)$ denote the set of 2-cells of $X$, and denote by $V=V(X)$ the set of vertices of $X$ and $E=E(X)$ the set of edges. 
An orientation of an edge $e$ is the choice of an oriented edge with image $e$. Note that each edge has two orientations. We choose once and for all an orientation for each edge of $X$, using the unique basic oriented edge with image $e$. If an oriented edge $\vec{e}$ with image $e$ is the chosen oriented edge we set $\eta(\vec{e})=1$, and if it is the opposite edge we set $\eta(\vec{e})=-1$.

We also choose once and for all for each basic oriented polygon $f: \pi_{\beta} \rightarrow X$ an orientation of each edges of $\partial \pi_{\beta}$, in such a way that the terminal point $v$ of an oriented edge of $\partial \pi_{\beta}$ is the initial point of the second oriented edge of $\partial \pi_{\beta}$ whose image contains $v$. For each vertex $v \in \partial \pi_{\beta}$ there is a unique cycle $\delta_{v} \pi_{\beta}=\left(\overrightarrow{e_{1}}, \ldots, \overrightarrow{e_{n}}\right)$ using the chosen oriented edges, with $i\left(\overrightarrow{e_{1}}\right)=v$, and such that $n$ is the number of sides of $\pi_{\beta}$. For each polygon $\pi$ of $X, \pi=f_{\beta}\left(\pi_{\beta}\right)$, and each vertex $v$ of $\pi_{\beta}$, we set $\delta_{v} \pi=\left(f_{\beta} \circ \overrightarrow{e_{1}}, \ldots, f_{\beta} \circ \overrightarrow{e_{n}}\right)$, where $\left(\overrightarrow{e_{1}}, \ldots, \overrightarrow{e_{n}}\right)=\delta_{v} \pi_{\beta}$.

Let $\mathbb{A}$ denote some finite abelian group. A 2-cocycle on $X$ (with values in $\mathbb{A}$ ) is just a map $c: \Pi \rightarrow \mathbb{A}$ assigning to each 2-cell $\pi$ of $X$ an element $c(\pi) \in \mathbb{A}$. A 1-cochain on $X$ (with values in $\mathbb{A}$ ) is a map $u: E \rightarrow \mathbb{A}$. The coboundary of a 1-cochain $u$ is the 2-cocycle $\delta u$ assigning to each 2-cell $\pi$ the sum $\sum \eta(\vec{e}) u(e)$ over $\vec{e} \in \delta_{v} \pi$. Note that this element of $\mathbb{A}$ is well-defined independently of the choice of $v$ on $\partial \pi_{\beta}$, because $\mathbb{A}$ is abelian (this is why we are using additive notation). The support of a 1 -cochain $u$ is the set of edges on which $u$ does not vanish; it will be denoted by $\operatorname{Supp}(u)$.

Let $\varphi$ denote an automorphism of $X$. For any edge $e$ we set $\eta(\varphi, e)=1$ whenever $\varphi$ sends the basic oriented edge at $e$ onto the basic oriented edge at $\varphi(e)$. Otherwise $\varphi$ sends the basic oriented edge at $e$ to the inverse of the basic oriented edge at $\varphi(e)$ and we set $\eta(\varphi, e)=-1$. For $u$ a 1-cochain and $e$ an edge we set $\varphi(u)(e)=$ $\eta\left(\varphi, \varphi^{-1}(e)\right) u\left(\varphi^{-1}(e)\right)$. We thus obtain an action of $\operatorname{Aut}(X)$ on the additive group of $1-$ cochains.

Similarly for any polygon $\pi$ of $X$ we set $\eta(\varphi, \pi)=1$ whenever $\varphi$ sends the basic oriented polygon at $\pi$ onto the basic oriented polygon at $\varphi(\pi)$. Otherwise we set $\eta(\varphi, \pi)=-1$. Then for any 2-cocycle $c$ and any polygon $\pi$ we set $\varphi(c)(\pi)$ equal to $\eta\left(\varphi, \varphi^{-1}(\pi)\right) c\left(\varphi^{-1}(\pi)\right)$. We thus obtain an action of $\operatorname{Aut}(X)$ on the additive group of 2-cocycles.

Example 8.11 Let $\varphi$ denote an automorphism of $X$. Let $\pi$ be a polygon of $X$ and let $\vec{e}$ denote some oriented edge of $\delta_{v} \pi$ for any vertex $v \in \partial \pi$. Set $\pi^{\prime}=\varphi(\pi)$, $v^{\prime}=\varphi(v)$ and let $\vec{e}$ be the oriented edge of $\delta_{v^{\prime}} \pi^{\prime}$ which has the same image as $\varphi \circ \vec{e}$. Then

$$
\eta\left(\overrightarrow{e^{\prime}}\right)=\eta(\varphi, \pi) \eta(\varphi, e) \eta(\vec{e})
$$

Algebraic 83 Geometric Topology, Volume 6 (2006) 
In particular we have $(\varphi(c)+\delta(\varphi u))\left(\pi^{\prime}\right)=\eta(\varphi, \pi)((c+\delta u)(\pi))$ for any 2-cocycle $c$ and any 1-cochain $u$. Also $\delta(\varphi u)=\varphi(\delta u)$.

\section{Walls}

Definition 9.1 Let $X$ denote some polygonal complex, and let $\pi$ denote some polygon in $X$, not a triangle. We say that two distinct oriented edges $\vec{a}, \vec{a}^{\prime}$ contained in $\pi$ are parallel in $\pi$ whenever there are disjoint connected subgraphs $\sigma, \sigma^{\prime}$ of $\partial \pi$ satisfying the following:

(1) the boundary $\partial \pi$ is the union $a \cup \sigma \cup a^{\prime} \cup \sigma^{\prime}$

(2) if $\ell, \ell^{\prime}$ denote the numbers of edges of $\sigma, \sigma^{\prime}$, then $\ell \leq \ell^{\prime}<\ell+2$

(3) the terminal vertices of the oriented edges $\vec{a}, \vec{a}^{\prime}$ belong to $\sigma$, so their initial vertices belong to $\sigma^{\prime}$.

If such subgraphs exist then they are unique. Hence any oriented edge $\vec{a}$ of a polygon $\pi$ is parallel to exactly one other oriented edge $\vec{a}^{\prime}$ of $\pi$. We denote by $\|$ the equivalence relation on oriented edges generated by parallelism inside polygons. Note that the group $\operatorname{Aut}(X)$ preserves the relation $\|$.
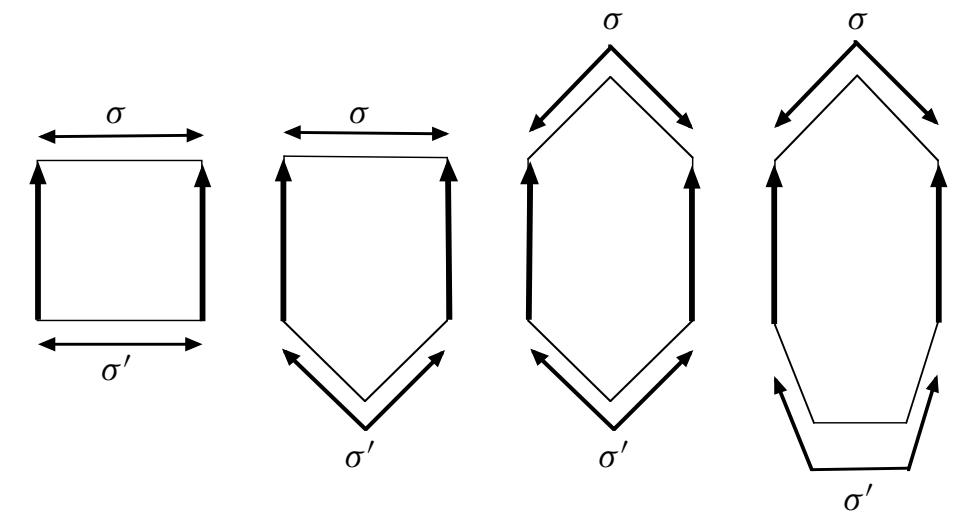

Figure 4: Two parallel oriented edges inside a polygon

Example 9.2 Let $k$ denote the number of edges of the polygon $\pi$, and assume $k \geq 4$. We consider parallelism inside $\pi$.

If $k$ is even then two oriented edges $\vec{a}, \vec{a}^{\prime}$ contained in $\pi$ are parallel in $\pi$ whenever they induce opposite orientations on $\partial \pi$ and the associated geometric edges $a, a^{\prime}$ are 
opposite on $\partial \pi$. Note that this is the parallelism relation defined in [26] for square complexes. See also Haglund and Paulin [23] and Wise [42].

If $k$ is odd, and so $k=2 t+1$, then $\ell=t-1$ and $\ell^{\prime}=t$. Thus two oriented edges $\vec{a}, \vec{a}^{\prime}$ contained in $\pi$ are parallel in $\pi$ whenever they induce opposite orientations on $\partial \pi$ and $i\left(\vec{a}^{\prime}\right)$ is the vertex of $\pi$ opposite to the center of $\vec{a}$.

Definition 9.3 (Walls) Let $X$ denote a polygonal complex. A wall of $X$ is an equivalence class of $\|$.

We say that a wall $M$ passes through an oriented edge $\vec{a}$ whenever $\vec{a} \in M$. We say that a wall $M$ separates a polygon $\pi$ of $X$ whenever $M$ passes through distinct oriented edges $\vec{a}, \vec{a}^{\prime}$ with $a \subset \partial \pi, a^{\prime} \subset \partial \pi$ and $\vec{a}, \vec{a}^{\prime}$ are parallel inside $\pi$.

A diameter of a polygon $\pi$ of $X$ is the union of two edges $[v, p],\left[v^{\prime}, p\right]$ of $X^{\prime}$ such that $p$ is the center of $\pi$, and there are distinct oriented edges $\vec{a}, \vec{a}^{\prime} \subset \partial \pi$ which are parallel in $\pi$, and whose centers are $v, v^{\prime}$. The diameter is then said to be dual to $\vec{a}, \vec{a}^{\prime}$.

The geometric wall associated to a wall $M$ is the subcomplex $|M|$ of the first barycentric subdivision $X^{\prime}$, union of those diameters of polygons which are dual to $\vec{a}, \vec{a}^{\prime}$, with $\vec{a}, \vec{a}^{\prime} \in M$.

If some wall $M$ passes through an oriented edge $\vec{a}$, we will also say that $M$ is dual to $\vec{a}$, and that $M$ is dual to $a$. For any wall $M$ we will denote by $V(M)$ the set of vertices $v$ belonging to an edge $a$ to which $M$ is dual.

Note that a combinatorial map $f: X \rightarrow Y$ maps a wall of $X$ inside a wall of $Y$. The same is true for the associated geometric wall.

Lemma 9.4 Assume that $X$ is locally compact, satisfies condition $\left(\mathrm{C}^{2}\right)$ and is simply connected. Equip $X$ with the $\left(\mathrm{C}^{2}\right)$-metric. Then:

(1) Each geometric wall is a totally geodesic subtree.

(2) Each geometric wall separates $X$ into two connected components.

(3) Two opposite oriented edges are not parallel.

(4) The intersection of a geometric wall and a polygon is either empty or a single diameter. In particular a geometric wall $|M|$ is the first barycentric subdivision of a tree whose vertices correspond to rank-1 vertices of $|M|$, and whose centers of edges correspond to rank-2 vertices of $|M|$. 


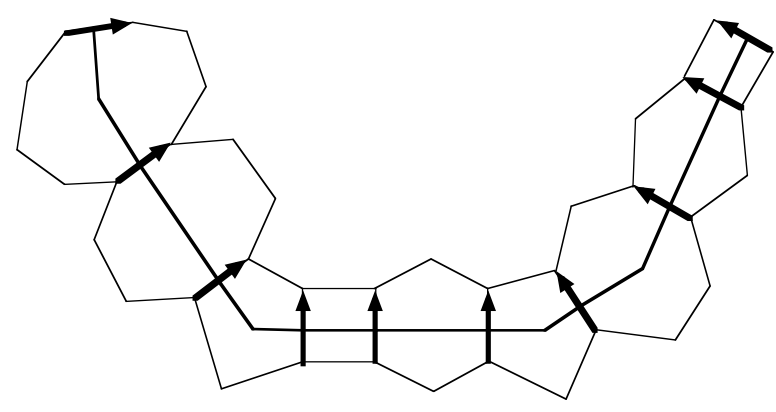

Figure 5: A wall and its associated geometric wall

Proof Consider any diameter $d=[v, p] \cup\left[p, v^{\prime}\right]$ of some polygon of $X$. By definition of the $\left(\mathrm{C}^{2}\right)$ metric the angle of the geodesic segments $[p, v],\left[p, v^{\prime}\right]$ at $p$ is $\pi$. Thus diameters of polygons are local geodesics.

Let $M$ denote some wall of $X$. By definition of $\|$, for any two points $x, y \in|M|$ there is a sequence of diameters $d_{0}, d_{1}, \ldots, d_{n}$ and a sequence of polygons $\pi_{0}, \pi_{1}, \ldots, \pi_{n}$ such that $d_{i} \subset \pi_{i}, x \in d_{0}, y \in d_{n}$, and $d_{i} \cap d_{i+1}$ contains a rank-1 vertex. After simplifications, we may assume that $d_{i} \neq d_{i+1}$. Then $d_{i} \cap d_{i+1}=\left\{v_{i+1}\right\}$, where $v_{i+1}$ is the center of an edge $\vec{a}_{i+1}$ to which $M$ is dual. Observe that the angle of $d_{i}$ and $d_{i+1}$ at $v_{i+1}$ is $\pi$, so that $\left(d_{i}, d_{i+1}\right)$ is a local geodesic.

It follows that $\left(d_{0}, d_{1}, \ldots, d_{n}\right)$ is a local geodesic. By Lemma 8.9 the $\left(\mathrm{C}^{2}\right)$ length metric on $X$ is $\operatorname{CAT}(0)$, so a local geodesic is a global one [8, Proposition 4.14, page 201].

We have shown that any two points of $|M|$ are contained in a geodesic segment of $X$ sitting in $|M|$. Thus $|M|$ is totally geodesic, and in particular it is contractible. Since $|M|$ is a subgraph of $X^{\prime}$, it follows that $|M|$ is a tree.

Since each diameter of a polygon $\pi$ is separating $\pi$, we deduce that $|M|$ is locally separating in two connected components. Since $|M|$ is a tree we see that $|M|$ separates a neighborhood in two connected components. But $X$ is simply connected, thus in fact $|M|$ separates $X$ in two connected components.

Assume now that $d, d^{\prime}$ are two distinct diameters of the same polygon $\pi$ of $X$. Then $d, d^{\prime}$ contain distinct edges $e, e^{\prime}$ of $X^{\prime}$ meeting at $p$ with angle less than $\pi$. In 
particular the convex hull of $d \cup d^{\prime}$ is not contained in the 1-skeleton of $X^{\prime}$. This shows that a totally geodesic union of diameters of $X$ (such as $|M|$ ) does not contain more than one diameter of each polygon.

Note that a parallelism between $\vec{a}$ and its opposite $\overleftarrow{a}$ creates a curve disjoint from $|M|$ and joining the endpoints of $\vec{a}$. This contradicts the fact that $|M|$ separates a neighborhood.

\section{Killing 2-cocycles}

\subsection{Groups acting without self-intersection}

Definition 10.1 (Self-intersecting wall) Let $X$ denote some simply connected polygonal complex and let $\Gamma$ denote some subgroup of $\operatorname{Aut}(X)$. We say that a wall $M$ is self-intersecting under $\Gamma$ whenever there exists $\gamma \in \Gamma$ such that $M, \gamma M$ are distinct, but the geometric walls associated to $M, \gamma M$ have nonempty intersection. We say that $\Gamma$ acts without self-intersection whenever no wall is self-intersecting under $\Gamma$.

If $X$ is a square complexes and $\Gamma$ acts freely, this definition is equivalent to the fact that $\Gamma \backslash X$ has no self-intersecting wall and no one-sided wall in the sense of [26].

Lemma 10.2 Let $\Gamma$ denote a discrete cocompact group of automorphism of some simply connected polygonal complex $X$. Assume that the stabilizers of walls are separable in $\Gamma$.

Then there is a finite index subgroup $\Gamma^{\prime} \subset \Gamma$ acting without self-intersection.

Proof Let $n$ denote the maximal number of sides of a polygon of the complex $X$.

For each wall $M$ of $X$ and each subgroup $G \subset \operatorname{Aut}(X)$ we introduce a set of bad elements:

$$
B(M, G)=\left\{g \in G, g M \neq M \text { and } \exists v, v^{\prime} \in V(M), d\left(v^{\prime}, g v\right) \leq n\right\}
$$

where $d$ denotes the combinatorial distance in the 1-skeleton.

Let $G_{M}$ denotes the stabilizer of $M$. Note that $G_{M}$ is the set of $g \in G$ such that there exists $\vec{e} \in M$ satisfying $g(\vec{e}) \in M$. Observe that $B(M, G)$ is right- and left-invariant under $G_{M}$, and contains those $g \in G$ such that $g M \neq M$ but $g|M| \cap|M| \neq \varnothing$.

There are finitely many $\Gamma$-orbits of oriented edges since $\Gamma$ is cocompact. Thus $\Gamma_{M} \backslash M$ and hence $\Gamma_{M} \backslash V(M)$ is finite. Thus there is a finite set $\left\{b_{1}, \ldots, b_{k}\right\}$ such 


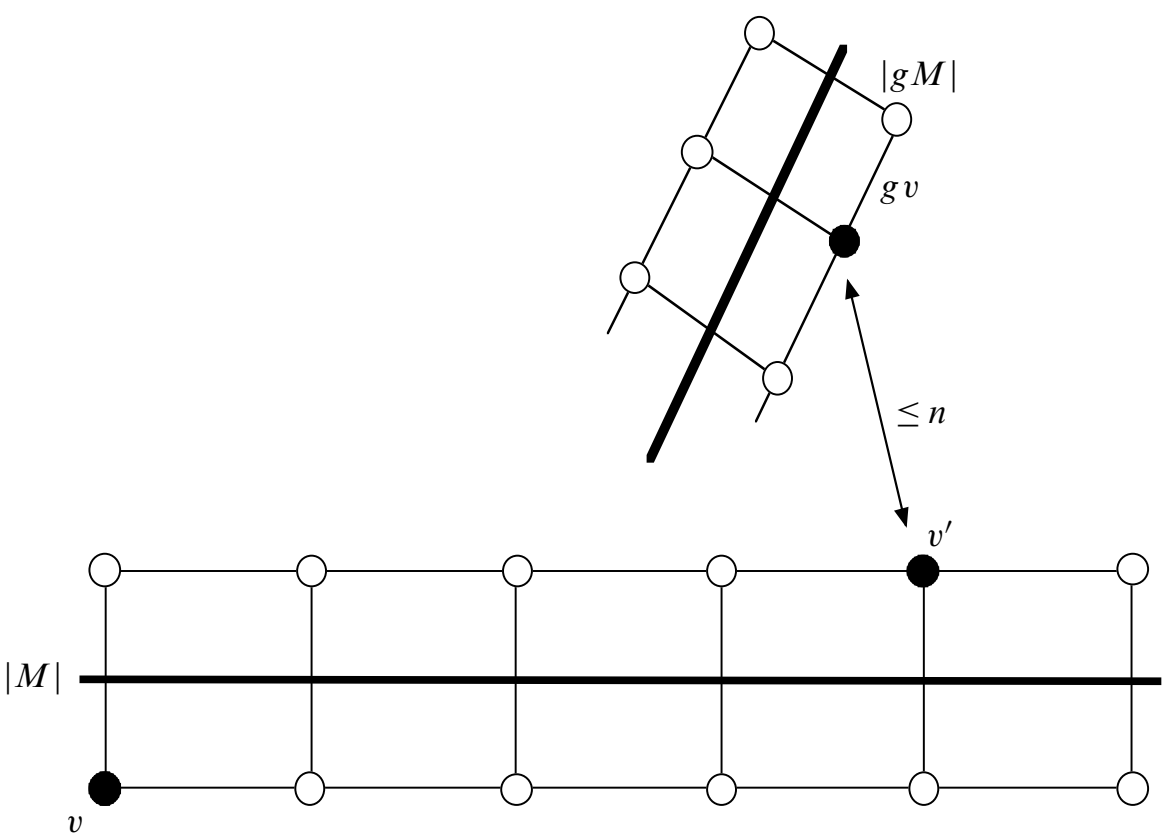

Figure 6: An element of $B(G, M)$

that $B(M, \Gamma)=\Gamma_{M} b_{1} \Gamma_{M} \cup \cdots \cup \Gamma_{M} b_{k} \Gamma_{M}$, since $X$ is locally compact and $\Gamma$ acts discretely.

By definition $\left\{b_{1}, \ldots, b_{k}\right\} \cap \Gamma_{M}=\varnothing$. Since $\Gamma_{M}$ is separable by assumption there is a finite index subgroup $\Gamma^{\prime} \subset \Gamma$ such that $\Gamma_{M} \subset \Gamma^{\prime}$ and $\left\{b_{1}, \ldots, b_{k}\right\} \cap \Gamma^{\prime}=\varnothing$. So for $1 \leq i \leq k$ we have $\Gamma^{\prime} \cap \Gamma_{M} b_{i} \Gamma_{M}=\varnothing$, hence $B\left(M, \Gamma^{\prime}\right)=B(M, \Gamma) \cap \Gamma^{\prime}=\varnothing$.

Let us consider any two translates $\gamma_{1} M, \gamma_{2} M\left(\gamma_{i} \in \Gamma^{\prime}\right)$ whose geometric associated walls have a nontrivial intersection. Then either $\gamma_{2}^{-1} \gamma_{1}$ stabilizes $M$ or it belongs to $B\left(M, \Gamma^{\prime}\right)$. The latter possibility has been excluded thus $\gamma_{1} M=\gamma_{2} M$.

So for the moment we have proved a weak version of the lemma: for each wall $M$ we have found a finite index subgroup $\Gamma^{\prime} \subset \Gamma$ such that any two translates $\gamma_{1} M, \gamma_{2} M$ $\left(\gamma_{i} \in \Gamma^{\prime}\right)$ either are equal, or have disjoint associated geometric walls.

If we consider a finite index subgroup $\Gamma^{\prime \prime} \subset \Gamma^{\prime}$ such that $\Gamma^{\prime \prime}$ is normal in $\Gamma$, then we will have the above property for each wall in the $\Gamma$-orbit of $M$.

Since $\Gamma$ is cocompact there are finitely many $\Gamma$-orbits of walls. Thus taking a finite intersection of finite index normal subgroups $\Gamma^{\prime \prime}$ as above yields a finite index subgroup with the desired property.

Algebraic ${ }^{6} \mathcal{G}$ Geometric Topology, Volume 6 (2006) 
When $X$ is assumed to be a square complex, a result similar to the previous lemma was proven in [26].

Remark 10.3 Let $G$ denote a group and let $H \subset G$ denote a subgroup all of whose finite index subgroups are separable in $G$. Then each finite index subgroup of $H$ is the intersection with $H$ of a finite index subgroup in $G$.

Indeed, let $K \subset H$ denote a finite index subgroup. Write $H=\cap_{i} G_{i}$ where each $G_{i}$ is a finite index subgroup of $G$. Then we also have $K=\cap_{i}\left(G_{i} \cap H\right)$. All subgroups $G_{i} \cap H$ are intermediate between $K$ and $H$. Since $K$ is of finite index in $H$ there are finitely many distinct intersections $G_{i} \cap H$. The corresponding intersection $\bigcap G_{i}$ yields a finite index subgroup of $G$ whose intersection with $H$ is $K$.

This applies in particular when $X$ is a simply connected polygonal complex and $\Gamma$ denotes a uniform lattice of $\operatorname{Aut}(X)$ such that each finite index subgroup of a wall stabilizer in $\Gamma$ is separable: each finite index subgroup of a wall-stabilizer in $\Gamma$ is the intersection with the wall-stabilizer of a finite index subgroup in $\Gamma$.

\subsection{Killing a 2-cocycle along a wall}

Theorem 10.4 Let $X$ denote some locally compact simply connected polygonal complex satisfying $\left(\mathrm{C}^{2}\right)$. Let $\Gamma^{0}$ denote a uniform lattice in $\operatorname{Aut}(X)$. Assume that $\Gamma^{0}$ has no self-intersection, and that each finite index subgroup of a wall-stabilizer contains the intersection with the wall-stabilizer of a finite index subgroup in $\Gamma^{0}$.

Let $\Gamma \subset \Gamma^{0}$ denote a finite index normal subgroup. Let $c: \Pi \rightarrow \mathbb{A}$ denote a $\Gamma$-invariant 2-cocycle on $X$.

For any wall $M$ there is a finite index normal subgroup $\Gamma^{\prime} \subset \Gamma^{0}$ and a $\Gamma^{\prime}$-invariant 2-cocycle $c^{\prime}$ on $X$ such that

(1) $c^{\prime}-c=\delta u$, where $u$ is a $\Gamma^{\prime}$-invariant 1-cochain

(2) if a polygon $\pi$ is not separated by any translate $\gamma M\left(\gamma \in \Gamma^{0}\right)$ then $c^{\prime}(\pi)=c(\pi)$

(3) if a polygon $\pi$ is separated by some (necessarily unique) translate $\gamma M\left(\gamma \in \Gamma^{0}\right)$ then $c^{\prime}(\pi)=0$.

Proof We first study the set $F=F(c, M)$ of 1-cochains $u$ with the following properties:

(1) $\operatorname{Supp}(u)$ is contained in the set of edges which are dual to $M$.

(2) for any polygon $\pi$ separated by $M$, if $\vec{e}$ and $\vec{e}$ are the two oriented edges of $\delta_{v} \pi$ such that $e$ and $e^{\prime}$ are dual to $M$ then $c(\pi)+u(e) \eta(\vec{e})+u\left(e^{\prime}\right) \eta\left(\overrightarrow{e^{\prime}}\right)=0$. (This condition does not depend on the choice of the vertex $v \in \partial \pi$.) 
In view of the first condition, note that the second condition reads $(c+\delta u)(\pi)=0$ for polygons $\pi$ that are separated by $|M|$. Note also that the pair of oriented edges $\vec{e}, \vec{e}$ of the second condition is well-defined because by Lemma 9.4, each geometric wall intersects $\pi$ in a single diameter.

Lemma 10.5 The set $F$ is finite, nonempty and invariant under the stabilizer $\Gamma_{M}$ of $M$ in $\Gamma$.

Proof To prove the two first assertions we consider an edge $e$ of $X$ dual to $M$, with center $v$, and we show that the map $F \rightarrow \mathbb{A}$ sending $u \in F$ to $u(e) \in \mathbb{A}$ is a bijection.

Consider a combinatorial injective path $c=\left(v_{0}=v, v_{1}, \ldots, v_{n}=w\right)$ of the tree $T(M)$ whose first barycentric subdivision is $|M|$ (see Lemma 9.4). Any edge $\left(v_{i-1}, v_{i}\right)$ of this path is contained in a single polygon $\pi_{i}$ of $X$. We denote by $\overrightarrow{e_{i}}$ the oriented edge of $\delta_{v} \pi_{i}$ whose center is $v_{i-1}$, and we denote by $\vec{f}_{i}$ the oriented edge of $\delta_{v} \pi_{i}$ whose center is $v_{i}$ (this well-defined independently of the choice of $v \in \partial \pi$ ). Observe that either $\overrightarrow{f_{i}}=\overrightarrow{e_{i+1}}$ or $\overrightarrow{f_{i}}=\overleftarrow{e_{i+1}}$

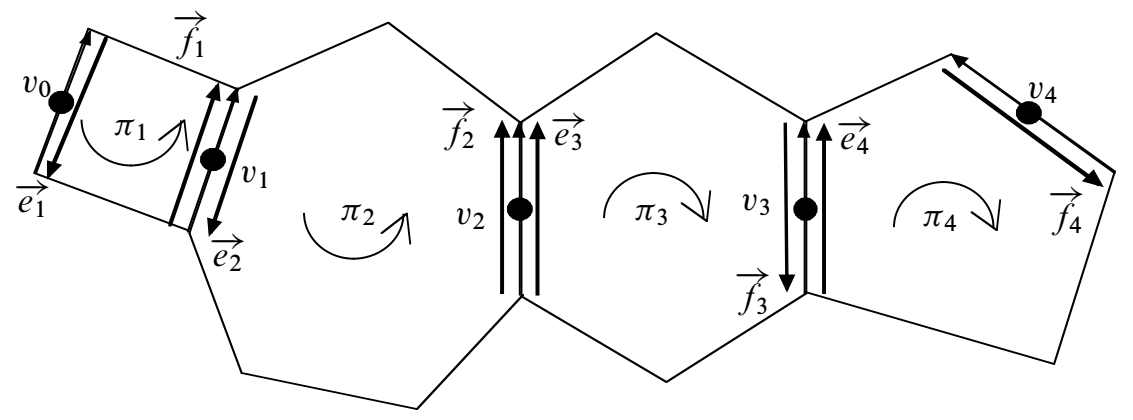

Figure 7: A path in $T(M)$. Each vertex $v_{i}$ is indicated by $\bullet$ and each center of an oriented edge of the wall $M$ is indicated with an arrow. The oriented edges $\vec{e}_{i}$ and $\vec{f}_{i}$ are drawn inside the polygons $\pi_{i}$.

Then we claim that for any element $g$ of $\mathbb{A}$, there exists one and only one sequence $\left(g_{0}, \ldots, g_{n}\right)$ of elements of $\mathbb{A}$ such that

(1) $g_{0}=g$

(2) for $1 \leq i \leq n$ we have $c\left(\pi_{i}\right)+g_{i-1} \eta\left(\overrightarrow{e_{i}}\right)+g_{i} \eta\left(\vec{f}_{i}\right)=0$.

Given $g_{i-1} \in \mathbb{A}$ the last equation is always solvable in the unknown $g_{i} \in \mathbb{A}$ because $\eta$ takes its values in $\{-1,+1\}$. So the claim follows by induction on the length $n$ of the path $c$. 
For any edge $e^{\prime}$ of $X$ dual to $M$, with center $w$, let $c=\left(v_{0}=v, v_{1}, \ldots, v_{n}=w\right)$ denote the unique injective combinatorial path in the tree $T(M)$ from $v$ to $w$. Using the notation above, we define $u\left(e^{\prime}\right)$ by setting $u\left(e^{\prime}\right)=g_{n}$. For any edge $e^{\prime \prime}$ not dual to $M$ we set $u\left(e^{\prime \prime}\right)=0$. We thus obtain the unique preimage of $g \in \mathbb{A}$ in $F$ under the restriction map $F \rightarrow \mathbb{A}$ (at the edge $e$ ).

It remains to check that $F$ is invariant under $\Gamma_{M}$. For $u \in F$ and $\gamma \in \Gamma_{M}$ clearly $\gamma u$ vanishes outside the set edges dual to $M$. Since $c$ is $\Gamma$-invariant, the calculations made in Example 8.11 show that the second relation defining elements of $F$ is still satisfied by $\gamma u$.

We construct $c^{\prime}, \Gamma^{\prime}$ using the action of $\Gamma_{M}$ on the sets $F(c, M)$.

Since $F(c, M)$ is finite and $\Gamma_{M}$ acts on it, a finite index subgroup $\Lambda \subset \Gamma_{M}$ fixes $F(c, M)$ pointwise. Since $\left[\Gamma_{M}^{0}: \Gamma_{M}\right]<+\infty$, there exists by assumption a finite index subgroup $\Gamma(c, M) \subset \Gamma^{0}$ such that $\Gamma(c, M) \cap \Gamma_{M} \subset \Lambda$, that is the wall-stabilizer $\Gamma(c, M)_{M}$ acts trivially on $F$. We may even assume that $\Gamma(c, M)$ is normal in $\Gamma^{0}$ and contained in $\Gamma$.

Consider all $\Gamma$-invariant 2-cocycles $d$. Observe that there are finitely many such $d$, because of $\Gamma$-invariance ( $\Gamma$ is cocompact). For each such $d$ we consider as above a finite index subgroup $\Gamma(d, M) \subset \Gamma$, which is normal in $\Gamma^{0}$, such that the stabilizer of $M$ in $\Gamma(d, M)$ acts trivially on $F(d, M)$. Then the intersection of all such $\Gamma(d, M)$ is a finite index normal subgroup $\Gamma^{\prime}$ of $\Gamma^{0}, \Gamma^{\prime} \subset \Gamma$, such that $\Gamma^{\prime}{ }_{M}$ acts trivially on all sets $F(d, M)$.

Choose elements $\gamma_{1}=1, \gamma_{2}, \ldots, \gamma_{m} \in \Gamma^{0}$ such that any wall $\gamma M$ (with $\gamma \in \Gamma^{0}$ ) is equivalent modulo $\Gamma^{\prime}$ to a unique $\gamma_{i} M=M_{i}$. We now define a $\Gamma^{\prime}$-invariant 1 -cochain $u$.

Pick elements $u_{i} \in F\left(c, M_{i}\right)$. We first check that $u_{i}$ is invariant under $\Gamma^{\prime} M_{i}$. Using the calculations made in Example 8.11, we see that $\gamma_{i}$ sends $F\left(\gamma_{i}^{-1} c, M\right)$ to $F\left(c, \gamma_{i} M\right)$. Observe that $\gamma_{i}^{-1} c$ is invariant under $\Gamma$ (because $\Gamma$ is normal in $\Gamma^{0}$ ). So by construction $\Gamma^{\prime} M$ fixes $F\left(\gamma_{i}^{-1} c, M\right)$. Thus $\gamma_{i} \Gamma^{\prime}{ }_{M} \gamma_{i}^{-1}$ fixes $F\left(c, \gamma_{i} M\right)$. But $\Gamma^{\prime}$ is normal in $\Gamma^{0}$, so $\gamma_{i} \Gamma^{\prime} M \gamma_{i}^{-1}=\Gamma^{\prime} M_{i}$.

For every $\Gamma^{0}$-translate $\gamma M, \gamma \in \Gamma^{0}$, we now choose some $\gamma^{\prime} \in \Gamma^{\prime}$ such that $\gamma^{\prime} \gamma_{i} M$ equals $\gamma M$ with $\gamma^{\prime}=1$ if $\gamma M$ is $\gamma_{i} M$ for some $i$.

For an edge $e$ not dual to a wall of the form $\gamma M$ for $\gamma \in \Gamma^{0}$, we set $u(e)=0$.

For an edge $e$ dual to $\gamma_{i} M$ we set $u(e)=u_{i}(e)$. Observe that by the absence of self-intersection $e$ is not dual to another $\gamma_{j} M$.

Algebraic 83 Geometric Topology, Volume 6 (2006) 
More generally if the edge $e$ is dual to a wall of the form $\gamma M$ for $\gamma \in \Gamma^{0}$ where $\gamma M=\gamma^{\prime} \gamma_{i} M$, we set $u(e)=\left(\gamma^{\prime} u_{i}\right)(e)$. Again $e$ is not dual to $\gamma^{\prime \prime} M$ not equal to $\gamma M$.

The 1-cochain $u$ is $\Gamma^{\prime}$-invariant because each $\Gamma^{\prime} M_{i}$ fixes $u_{i}$.

We set $c^{\prime}=c+\delta u$ : this new 2 -cocycle is $\Gamma^{\prime}$-invariant $\left(\Gamma^{\prime} \subset \Gamma\right)$.

We now compute $c^{\prime}(\pi)$ for some polygon $\pi$.

If $\pi$ is not separated by a wall of the form $\gamma M\left(\gamma \in \Gamma^{0}\right)$ then for each edge $e \subset \partial \pi$ we have $u(e)=0$. Thus $c^{\prime}(\pi)=c(\pi)$.

Assume now that $\pi$ is separated by at least one $\Gamma$-translate of $M$. Since distinct $\Gamma$-translates of $M$ have disjoint associated geometric walls, in fact there exists a unique translate $\gamma M$ which separates $\pi$. On the set of edges dual to $\gamma M$ the 1-cochain $u$ coincides with an element of $F(c, \gamma M)$. Thus $c^{\prime}(\pi)=0$.

The 2 -cocycle $c^{\prime}$ is trivial on a $\Gamma^{0}$-invariant set of polygons, and $c^{\prime}=c$ on the $\Gamma^{0}$-invariant complement of this set. Thus $c^{\prime}$ is $\Gamma$-invariant (but it is cohomologous to $c$ only by the coboundary of a $\Gamma^{\prime}$-invariant 1 -cochain).

\subsection{Killing a 2-cocycle in a finite cover}

Theorem 10.6 Let $X$ denote a compact polygonal complex satisfying the nonpositive curvature condition $\left(\mathrm{C}^{2}\right)$. Assume that finite index subgroups of wall stabilizers are separable in $\Gamma=\pi_{1}(X)$. Then for any 2-cocycle $c$ on $X$, there is a (regular) finite cover $p: X^{\prime} \rightarrow X$ such that the lift $p^{*}(c)$ is cohomologous to zero.

Proof First note that each basic oriented edge or polygon of $X$ lifts to the universal cover $\tilde{p}: \widetilde{X} \rightarrow X$. We thus endow $\widetilde{X}$ with a natural structure of polygonal complex such that $\tilde{p}$ is combinatorial and in fact sends basic oriented edges or polygons to basic oriented edges or polygons. Thus the deck transformation group $\Gamma$ also sends basic oriented edges or polygons to basic oriented edges or polygons. (In particular for any $\gamma \in \Gamma$ and any edge $e$ or any polygon $\pi$ we have $\eta(\gamma, e)=\eta(\gamma, \pi)=1$.)

First by Lemma 10.2 there is a finite index subgroup of $\Gamma$ acting without self-intersection. So we may and will assume that $\Gamma$ acts without self-intersection.

We denote by $\tilde{c}$ the 2 -cocycle on $\widetilde{X}$ preimage of $c$ under the universal cover $\widetilde{X} \rightarrow X$.

By construction $\tilde{c}$ is $\Gamma$-invariant.

We choose walls $M_{1}, \ldots, M_{n}$ such that any wall of $\widetilde{X}$ is a $\Gamma$-translate of a unique wall of the family $\left\{M_{1}, \ldots, M_{n}\right\}$.

Algebraic $8 \mathcal{G}$ Geometric Topology, Volume 6 (2006) 
We first set $\Gamma^{0}=\Gamma$. Using Remark 10.3, we see that we may apply Theorem 10.4 to the wall $M_{1}$. Thus we find a finite index normal subgroup $\Gamma^{1} \subset \Gamma$, a 1 -cochain $u^{1}$ and a 2-cocycle $c^{1}$ such that $u^{1}, c^{1}$ are $\Gamma^{1}$-invariant, $c^{1}=\tilde{c}+\delta u^{1}$, and for every polygon $\pi$, either $\pi$ is separated by no $\Gamma$-translate and $c^{1}=\tilde{c}$ on $\pi$, or $\pi$ is separated by some $\Gamma$-translate $\gamma M$ and then $c^{1}(\pi)=0$.

We apply again Theorem 10.4 to the subgroup $\Gamma^{1}$, the 2-cocycle $c^{1}$ and the wall $M_{2}$, thus getting a finite index normal subgroup $\Gamma^{2} \subset \Gamma$ with $\Gamma^{2} \subset \Gamma^{1}$, together with a 1-cochain $u^{2}$ and a 2-cocycle $c^{2}=c^{1}+\delta u^{2}$ which is trivial along $\Gamma$-translates of $M_{2}$. If we repeat this process until we reach the $\Gamma$-translates of $M_{n}$, we obtain a decreasing sequence of normal subgroups $\Gamma^{1}, \ldots, \Gamma^{n}$ (each of finite index in $\Gamma$ ), together with sequences of 1-cochains, $u^{1}, \ldots, u^{n}$ and 2-cocycles $c^{1}, \ldots, c^{n}$ with $u^{i}, c^{i}$ invariant under $\Gamma^{i}$, and $c^{i+1}=c^{i}+\delta u^{i+1}$.

We claim that $c^{n}=0$.

Indeed consider a polygon $\pi$ with $k$ edges. The walls separating $\pi$ are in the $\Gamma$-orbit of $M_{i_{1}}, \ldots, M_{i_{k}}$ with $i_{1}<\cdots<i_{k}$. We denote by $N_{j}$ the $\Gamma$-translate of $M_{i_{j}}$ which separates $\pi$.

For $i \notin\left\{i_{1}, \ldots, i_{k}\right\}$ we know that $c^{i}=c^{i-1}$ on $\pi$ (by convention $c^{0}=\tilde{c}$ ), and for $i \in\left\{i_{1}, \ldots, i_{k}\right\}$ we know that $c^{i}(\pi)=0$. Thus $c^{n}(\pi)=0$.

Let $p: X^{\prime} \rightarrow X$ denote the (regular) covering corresponding to the normal subgroup $\Gamma^{n} \subset \pi_{1}(X)$. The lift $p^{*}(c)$ lifts to $\widetilde{c}$ in $\widetilde{X}$. Note that $\tilde{c}, u^{1}, \cdots, u^{n}$ are all invariant under $\Gamma^{n}$. Thus each $c^{i}$ is $\Gamma^{n}$-invariant and defines a 2-cocycle $c_{i}$ on $X^{\prime}$. Furthermore $c_{i}$ is cohomologous to $c_{i-1}$ on $X^{\prime}$. As a conclusion $p^{*}(c)=c_{0}$ is cohomologous to $c_{n}=0$.

Theorem 10.7 Let $X$ denote a compact polygonal complex satisfying the nonpositive curvature condition $\left(\mathrm{C}^{2}\right)$. Assume that finite index subgroups of wall-stabilizers are separable in $\Gamma=\pi_{1}(X)$.

Then for any extension $1 \rightarrow G \rightarrow \bar{\Gamma} \rightarrow \Gamma \rightarrow 1$ where $G$ is finite, the group $\bar{\Gamma}$ is commensurable with $\Gamma$.

Proof Let $\pi: \bar{\Gamma} \rightarrow \Gamma$ denote a morphism whose finite kernel is $G$.

Let us denote by $Z$ the centralizer of $G$ in $\bar{\Gamma}$. Since $G$ is normal and finite $Z$ is a finite index subgroup of $\bar{\Gamma}$. So $\pi(Z)$ is a finite index subgroup of $\Gamma$ and the kernel of $\pi: Z \rightarrow \pi(Z)$ is central in $Z$. This means that we may restrict ourselves to central extension $1 \rightarrow \mathbb{A} \rightarrow \bar{\Gamma} \rightarrow \Gamma \rightarrow 1(G=\mathbb{A}$ is now a finite abelian group and $\mathbb{A}$ is contained in the center of $\bar{\Gamma}$ ).

Algebraic 83 Geometric Topology, Volume 6 (2006) 
Central extensions of $\Gamma$ by $A$ correspond, up to isomorphisms of extensions, to cohomology classes of $H^{2}(\Gamma, A)$ ( $\Gamma$ acts trivially on $A$ ) (see Brown [9, Theorem 3.12, page 93]). Since $\widetilde{X}$ is CAT(0) it is contractible, $X$ is an Eilenberg-MacLane space for $\Gamma$. Thus $H^{2}(\Gamma, \mathbb{A}) \simeq H^{2}(X, \mathbb{A})$, the group of 2-cocycles on $X$ modulo the subgroup of coboundaries of 1 -cocycles, and the product extension $1 \rightarrow \mathbb{A} \rightarrow \mathbb{A} \times \Gamma \rightarrow \Gamma \rightarrow 1$ corresponds to the trivial cohomology class. For convenience we recall here very briefly the identification between the set of central extensions of $\Gamma$ by $\mathbb{A}$ and the group $H^{2}(X, \mathbb{A})$.

Suppose first we are given some central extension $1 \rightarrow \mathbb{A} \rightarrow \bar{\Gamma} \rightarrow \Gamma \rightarrow 1$. The inclusion $\left(X^{1}, x\right) \rightarrow(X, x)$ induces a surjective map $F=\pi_{1}\left(X^{1}, x\right) \rightarrow \Gamma=\pi_{1}(X, x)$. The kernel $R$ of this map is the subgroup generated by conjugates of elements $\gamma_{\pi}$ in $\pi_{1}\left(X^{1}\right)=\sigma_{\pi} \cdot \delta_{v} \cdot \pi \sigma_{\pi}{ }^{-1}$ where $\pi$ denote any polygon of $X$, and $\sigma_{\pi}$ is a path in $X^{1}$ from the basepoint $x$ to some chosen vertex $v$ of $\partial \pi$. Since $F$ is free and $\bar{\Gamma} \rightarrow \Gamma$ is onto, the quotient morphism $F \rightarrow \Gamma$ has a lift to $\bar{\Gamma}$. Choose any lift $c: F \rightarrow \bar{\Gamma}$ of $F \rightarrow \Gamma$. Any element $r \in R$ maps to an element $c(r) \in \mathbb{A}$ under this lift. In particular we may associate to any polygon $\pi$ of $X$ an element $c(\pi)=c\left(\gamma_{\pi}\right) \in \mathbb{A}$ (independent of the choice of $\sigma_{\pi}$ because $\mathbb{A}$ is central). A different choice of lift leads to a cohomologous 2-cocycle. Note that $c=0$ means that the morphism $c: F \rightarrow \bar{\Gamma}$ is trivial on $K$, thus defines a section of $\bar{\Gamma} \rightarrow \Gamma$. Then $\bar{\Gamma}$ is a semidirect product, hence a direct product since $\mathbb{A}$ is central.

Conversely let $c$ denote some 2-cocycle on $X$ with values in $\mathbb{A}$. In the free product $\mathbb{A} * F$ consider the normal subgroup $N$ generated by the elements $c\left(\gamma_{\pi}\right)^{-1} \gamma_{\pi}$ and the commutators $[g, f], g \in \mathbb{A}, f \in F$. Set $\bar{\Gamma}=F / N$. Extend trivially on $\mathbb{A}$ the natural map $F \rightarrow \Gamma$, then $\mathbb{A} * F \rightarrow \Gamma$ defines a surjective morphism $\bar{\Gamma} \rightarrow \Gamma$. Furthermore the natural inclusion $\mathbb{A} \rightarrow \mathbb{A} * F$ defines an inclusion $\mathbb{A} \rightarrow \bar{\Gamma}$ whose image is central. We have an exact sequence $1 \rightarrow \mathbb{A} \rightarrow \bar{\Gamma} \rightarrow \Gamma \rightarrow 1$, whose 2-cocycle is $c$. A cohomologous $2-$ cocycle $c^{\prime}$ leads to an isomorphic central extension.

Given a central extension $1 \rightarrow \mathbb{A} \rightarrow \bar{\Gamma} \rightarrow \Gamma \rightarrow 1$, let $c$ denote the associated 2cocycle on $X$. By Theorem 10.6, there is a regular finite cover $p: X^{\prime} \rightarrow X$ such that $p^{*}(c)$ is cohomologous to 0 . Let $\Gamma^{\prime}$ denote the finite index normal subgroup of $\Gamma$ corresponding to the covering $X^{\prime} \rightarrow X$. Let $\overline{\Gamma^{\prime}}$ denote the preimage of $\Gamma^{\prime}$ under $\bar{\Gamma} \rightarrow \Gamma$. Then $p^{*}(c)$ is the 2-cocycle on $X^{\prime}$ corresponding to the central extension $1 \rightarrow \mathbb{A} \cap \overline{\Gamma^{\prime}} \rightarrow \overline{\Gamma^{\prime}} \rightarrow \Gamma^{\prime} \rightarrow 1$. This latter extension is trivial, hence $\overline{\Gamma^{\prime}}$ contains a finite index subgroup mapping isomorphically onto $\Gamma^{\prime}$.

Proof of Theorem 1.10 It suffices to note that if $X$ satisfies $\left(\mathrm{C}^{2}\right)$ the geometric walls are convex subtrees by Lemma 9.4. Thus the wall-stabilizers and their finite index 
subgroups are convex subgroups, hence separable by assumption. It remains to apply Theorem 10.7.

\section{Part III Commensurability in some Coxeter-Davis complexes}

In the first part of this paper we studied type-preserving uniform lattices of the rightangled building associated with a graph product of finite groups. In this part we consider a two-dimensional Coxeter group, and we are interested in the uniform lattices of the polygonal complex associated with the Coxeter group. The results of this part are quite similar to the results of part 1 , the method is rather analogous to the method used in part 2.

In all of this part we will denote by $L$ some simplicial graph. We denote by $I$ the set of vertices of $L$ and by $E$ the set of its edges. We let $\mu$ denote the girth of $L$, that is the least length of a simple closed combinatorial path in $L$. We consider a map $m$ assigning to each edge $e$ of $L$ an integer $m(e) \geq 2$.

We consider the Coxeter presentation

$$
\left.\left\langle s_{i}, i \in I\right| s_{i}^{2}=1, \forall i \in I ;\left(s_{i} s_{j}\right)^{m(e)}=1, \forall e \in E \text { with } \partial e=\{i, j\}\right\rangle
$$

and denote by $(W, S)$ the associated Coxeter system where $W$ is the group and $S$ is the generating set $\left\{s_{i}, i \in I\right\}$. For $i, j \in J, i \neq j$ we set $m_{i j}=m(e)$ if there exists an edge $e \in E$ with $\partial e=\{i, j\}$, and otherwise we set $m_{i j}=\infty$. For any subset $J \subset I$ we denote by $W_{J}$ the subgroup of $W$ generated by $\left\{s_{j}\right\}_{j \in J}$. We recall that the natural map

$\left\langle s_{i}, i \in J\right| s_{i}^{2}=1, \forall i \in J ;\left(s_{i} s_{j}\right)^{m(e)}=1, \forall e \in E$ with $\left.\partial e=\{i, j\}, i \in J, j \in J\right\rangle \rightarrow W_{J}$ is an isomorphism, so $\left(W_{J}, J\right)$ is also a Coxeter system. For this and other general results on Coxeter systems we refer to Bourbaki [5].

\section{Coxeter-Davis complexes}

In this section we associate to the Coxeter system a geometric realization. As a building block we first introduce a simplicial complex of dimension two: $B_{*}=x_{*} * L^{\prime}$. So $B_{*}$ is obtained as the join of a single vertex $x_{*}$ with the first barycentric subdivision of $L$. We set $\partial B_{*}=L^{\prime} \subset B_{*}$. The facets of $B_{*}$ are the subcomplexes $\phi_{i}$, stars of $i$ in $\partial B_{*}$. Note that the facets cover $\partial B_{*}$. For each point $p \in B_{*}$ let $\tau(p)$ denote the 
smallest simplex of $B_{*}$ containing $p$. We then define $t(p) \subset I$ as the set of $i \in I$ such that $\tau(v) \subset \phi_{i}$. Now we define an equivalence relation $\sim$ on $W \times B_{*}$ by declaring $(w, p) \sim\left(w^{\prime}, p^{\prime}\right) \Longleftrightarrow p=p^{\prime} \in \partial B_{*}$ and $w^{-1} w^{\prime} \in W_{t(p)}$

We will denote by $A=A\left(W, B_{*}\right)$ the quotient of $W \times B_{*}$ by $\sim$ and we will denote by $[w, p]$ the image in $A$ of $(w, p)$. Note the analogies with the first part: $\mathcal{G} \leftrightarrow L, \Gamma \leftrightarrow W, C_{*} \leftrightarrow B_{*}, \Delta \leftrightarrow A$.

In the following proposition we list some well-known properties of $A$ [19] and we introduce some notation.

Proposition 11.1 (1) The left action of $W$ on $W \times B_{*}$ induces an action of $W$ on A.

(2) The second projection $W \times B_{*} \rightarrow B_{*}$ induces a $W$-invariant map $\rho: A \rightarrow B^{*}$, and $\rho(p)=\rho(q)$ if and only if $p, q$ belong to the same $W$-orbit.

For $p \in A$ we set $t(p)=t(\rho(p))$ (the type of $p$ ) and $\operatorname{rk}(p)=|t(p)|$ (the rank of $p$ ).

(3) The map $j$ sending $p \in B^{*}$ to $[1, p]$ satisfies $\rho j=\mathrm{id}_{B^{*}}$. Thus we will identify $B^{*}$ with its image $j\left(B^{*}\right)$, and $B^{*}$ is then a strict fundamental domain for the action of $W$ on $A$ (meaning that each $W$-orbit intersects $B^{*}$ in a single point).

(4) The quotient $A$ admits a unique structure of simplicial complex such that the natural map $W \times B_{*} \rightarrow A$ is combinatorial. The $W$-translates $w B^{*}$ are the blocks of $A$, and for any facet $\phi_{i}$ of $B^{*}$ with center $v$ and any $w \in W$ we call $w \phi_{i}$ a facet of $A$ with center $w v$. The vertex $w x_{*} \in w B_{*}$ is called the center of the block $w B_{*}$.

For $J \subset\{0,1,2\}$ we will denote by $A^{J}$ the full subcomplex of $A$ whose vertices $v$ satisfy $t(v) \in J$.

(5) For each $p \in B_{*}$ the stabilizer of $p$ in $W$ is $W_{t(p)}$. In particular $W$ acts simply transitively on the blocks of $A$. If two distinct blocks share a rank-1 vertex $v$, then their intersection is the facet with center $v$.

(6) Let $v$ denote a vertex of $A$ of type $J$ and rank $r$. Then $r \leq 2$. If $r=0$ then $v$ is the center of a unique block of $A$, and $\operatorname{link}(v, A)$ is isomorphic to $\partial B^{*}$. If $r=1$ then $v$ is the center of a unique facet of $A$, and $\operatorname{link}(v, A)$ is the suspension of the boundary of the facet. If $r=2$ then $J=\{i, j\}$ with $m_{i j}<\infty$ and $\operatorname{link}(v, A)$ is a cyclic graph with $m_{i j}$ vertices of type $\{i\}, m_{i j}$ vertices of type $\{j\}$ and $2 m_{i j}$ vertices of type $\varnothing$.

(7) There exists a unique regular polygonal complex $X=X\left(W, B_{*}\right)$ whose barycentric subdivision is $A$ in such a way that the rank of the center of a face of $X$ is the dimension of the face, that is, the rank in $A$ coincides with the rank of 
barycentric subdivisions. For any vertex $x$ of $X$ we will denote by $B(x)$ the block of $A$ with center $x$.

(8) $A$ is simply connected.

Remark 11.2 By Remark 8.5, the group $\operatorname{Aut}_{\mathrm{rk}}(A)$ of combinatorial automorphisms of $A$ preserving the rank in fact coincides with $\operatorname{Aut}(X)$.

Definition 11.3 The Coxeter system $(W, S)$ is two-dimensional whenever $W_{J}$ is infinite for each subset $J \subset I$ with $|J| \geq 3$.

Lemma 11.4 (1) If the Coxeter system $(W, S)$ is two-dimensional then the polygonal complex $X$ satisfies the nonpositive curvature condition $\left(\mathrm{C}^{2}\right)$.

(2) If the Coxeter system $(W, S)$ is two-dimensional and I does not contain any subset $\{i, j, \ell\}$ with $m_{i j} \leq 3, m_{j \ell}=3, m_{\ell i}<+\infty$, then $X$ satisfies the condition $\left(\mathrm{C}^{4}\right)$.

(3) If the girth $\mu$ of $L$ is at least 4 then $(W, S)$ is two-dimensional and $X$ satisfies condition $(Q)$.

Proof (1) In the case of $X$, the nonpositive curvature condition $\left(\mathrm{C}^{2}\right)$ reads as follows:

$$
\text { If } c=\left(\overrightarrow{e_{1}}, \ldots, \overrightarrow{e_{n}}\right) \text { is a cycle of } L \text { then } \sum_{i}\left(\frac{1}{2}-\frac{1}{2 m_{e_{i}}}\right) \geq 1 \text {. }
$$

Since $m \geq 2$ this condition is obviously fulfilled for any cycle of length $n \geq 4$. So assume that $c$ has length 3 . Let $J$ denote the set of vertices of $L$ belonging to one of the edge of $c$. The subgroup $W_{J}$ must be infinite by the condition on dimension. But $W_{J}$ is a triangle group, so being infinite implies $1 / m\left(e_{1}\right)+1 / m\left(e_{2}\right)+1 / m\left(e_{3}\right) \leq 1$, which is equivalent to $\left(\mathrm{C}^{2}\right)$ in this particular case.

(2) The condition $\left(\mathrm{C}^{4}\right)$ reads as follows:

$$
\text { If } c=\left(\overrightarrow{e_{1}}, \ldots, \overrightarrow{e_{n}}\right) \text { is a cycle of } L \text { then } \sum_{i}\left(\frac{1}{2}-\frac{1}{4 f_{e_{i}}}\right) \geq 1
$$

where $4 f\left(e_{i}\right)=2 m\left(e_{i}\right)$ if $m\left(e_{i}\right)$ is even, and $4 f\left(e_{i}\right)=2 m\left(e_{i}\right)-2$ otherwise.

Again this relation is obviously fulfilled for any cycle of length $n \geq 4$. So assume that $c=\left(\overrightarrow{e_{1}}, \overrightarrow{e_{2}}, \overrightarrow{e_{3}}\right)$ has length 3 . Let $J$ denote the set of vertices of $L$ belonging to one of the edges of $c$. We may assume that $m\left(e_{1}\right) \leq m\left(e_{2}\right) \leq m\left(e_{3}\right)$. Necessarily $m\left(e_{3}\right)>3$, otherwise the group $W_{J}$ is either finite commutative or has one $m\left(e_{i}\right)$ equal to 3 , another $m\left(e_{i}\right)$ at least 3 and the last one is finite. Similarly $m\left(e_{2}\right)>2$, 
otherwise the group $W_{J}$ is finite, and in fact $m\left(e_{2}\right)>3$ by the hypotheses. Then $\sum_{i}\left(1 / 2-1 /\left(4 f_{e_{i}}\right)\right) \geq(1 / 2-1 / 4)+(1 / 2-1 / 8)+(1 / 2-1 / 8)=1$.

(3) If the girth $\mu$ of $L$ is $\geq 4$ then in any subset $\{i, j, \ell\} \subset I$ one of the quantities $m_{i j}, m_{j \ell}, m_{\ell i}$ is infinite, hence $(W, S)$ is two-dimensional. Condition $(\mathrm{Q})$ is trivially fulfilled.

Example 11.5 Assume that the function $m: I \rightarrow\{2,3, \ldots\}$ is constant. Then the polygonal complex $X$ in Proposition 11.1 is the complex $X(m, L)$ of Section 1 . The condition " $m \geq 3$ or $m=2$ and the girth of $L$ is at least 4 " is equivalent to $(W=W(m, L), S)$ being two-dimensional.

When this condition is fulfilled by Lemma 11.4, we see that $X$ satisfies the condition $\left(\mathrm{C}^{2}\right)$, hence also the (more natural) nonpositive curvature condition $(\mathrm{C})$ : equipped with a piecewise Euclidean length metric turning each polygon to a regular Euclidean polygon, $X$ is $\operatorname{CAT}(0)$.

Observe that the condition " $X(m, L)$ is negatively curved" of Section 1 , that is, $m \geq 4$, or $m \geq 3$ and the girth of $L$ is at least 4 , or $m \geq 2$ and the girth of $L$ is at least 5 , is stronger than the two-dimensionality condition. Furthermore it is clear that under these hypotheses, $I$ does not contain any subset $\{i, j, \ell\}$ with $m_{i j} \leq 3, m_{j \ell}=3, m_{\ell i}<+\infty$. So condition $\left(\mathrm{C}^{4}\right)$ is satisfied. In fact the condition is equivalent to condition $\left(\mathrm{C}^{4}\right.$ ') for complexes $X(m, L))$. Thus by Lemma 8.9 the terminology "negatively curved" is justified.

\section{Reflections, walls and $e$-walls}

Definition 12.1 (Reflections) The reflections of $W$ are the conjugate of the generating involutions $s_{i}, i \in I$. The fixed point set of a reflection $r=w s_{i} w^{-1}$ on $A$ is the geometric wall of $r$, denoted by $M(r)$.

Lemma 12.2 The geometric wall of a reflection $r$ is the geometric wall associated to some wall of the polygonal complex $X$ (see Definition 9.3). The geometric wall of a reflection $r$ is a separating totally geodesic subtree of $A$.

Proof Since $W$ acts simply transitively on vertices of $X$, the fixed point set $M(r)$ is contained in the graph $A^{\{1,2\}}$. Let us check that for any polygon $\pi$ of $X$ either $\pi \cap M(r)=\varnothing$ or $\pi \cap M(r)$ is a diameter of $\pi$ (see Definition 9.3). Note that $M(r)$ is the fixed point set of an isometry of $C$, hence it is a convex subcomplex of the CAT(0) complex $A\left[8\right.$, Proposition 6.2]. Thus $M(r)$ is a subtree of $A^{\{1,2\}}$. Each 
generating reflection $s_{i} \in S$ fixes the unique vertex of type $\{i\}$ in $B_{*}$, and $s_{i}$ preserves each polygon of $X$ containing this vertex. In particular $M(r)$ contains a vertex of rank 1.

Assume that $\pi \cap M(r) \neq \varnothing$. Then since $M(r)$ is connected and contains a rank1 vertex, the intersection $\pi \cap M(r)$ also contains a rank-1 vertex. Observe that there is a reflection $r^{\prime}$ which fixes the same rank-1 vertex and furthermore preserves $\pi$. Necessarily $r=r^{\prime}$, thus $r$ preserves $\pi$. The restriction of $r$ to $\pi$ is an affine automorphism preserving an edge and fixing no vertex: thus $r$ acts on $\pi$ like a usual reflection, and $\pi \cap M(r)$ is a diameter of $\pi$.

It follows easily that $M(r)$ is union of geometric walls, hence a single geometric wall by connectedness. The rest of the lemma follows by Lemma 9.4 .

For later purposes we have to consider other types of walls. We want walls to be (separating) CAT(0)-subtrees of $A^{\{1,2\}}$, but we also want a parity condition that the wall of a reflection might fail to satisfy.

Definition 12.3 Let $X$ denote some polygonal complex, and let $\pi$ denote some polygon in $X$, not a triangle. We say that two oriented edges $\vec{a}, \vec{a}^{\prime}$ contained in $\pi$ are even-parallel in $\pi$ whenever there are disjoint connected subgraphs $\sigma, \sigma^{\prime}$ of $\partial \pi$ satisfying the following holds:

(1) The boundary $\partial \pi$ is the union $a \cup \sigma \cup a^{\prime} \cup \sigma^{\prime}$.

(2) If $\ell, \ell^{\prime}$ denote the numbers of edges of $\sigma, \sigma^{\prime}$, then $\ell$ is odd and $\ell \leq \ell^{\prime}<\ell+4$.

(3) The terminal vertices of the oriented edges $\vec{a}, \vec{a}^{\prime}$ belong to $\sigma$ (so their initial vertices belong to $\sigma^{\prime}$ ).

Observe that if such subgraphs exist then they are unique. Hence any oriented edge $\vec{a}$ of a polygon $\pi$ is even-parallel to exactly one other oriented edge $\vec{a}^{\prime}$ of $\pi$. We denote by $\|_{e}$ the equivalence relation on oriented edges generated by even-parallelism inside polygons: that is we write $\vec{a} \|_{e} \vec{a}^{\prime}$ whenever there is a sequence of polygons $\pi_{1}, \ldots, \pi_{n}$ and a sequence of oriented edges $\vec{a}_{0}=\vec{a}, \ldots, \vec{a}_{n}=\vec{a}^{\prime}$ such that $\vec{a}_{i}$ and $\vec{a}_{i+1}$ are even-parallel in $\pi_{i+1}$. Note that the group $\operatorname{Aut}(X)$ preserves the relation $\| e$.

Example 12.4 Let $k \geq 4$ denote the number of edges of the polygon $\pi$.

If $4 \mid k$ then two oriented edges $\vec{a}, \vec{a}^{\prime}$ contained in $\pi$ are even-parallel in $\pi$ whenever they induce opposite orientations on $\partial \pi$ and the associated geometric edges $a, a^{\prime}$ are opposite on $\partial \pi$. 


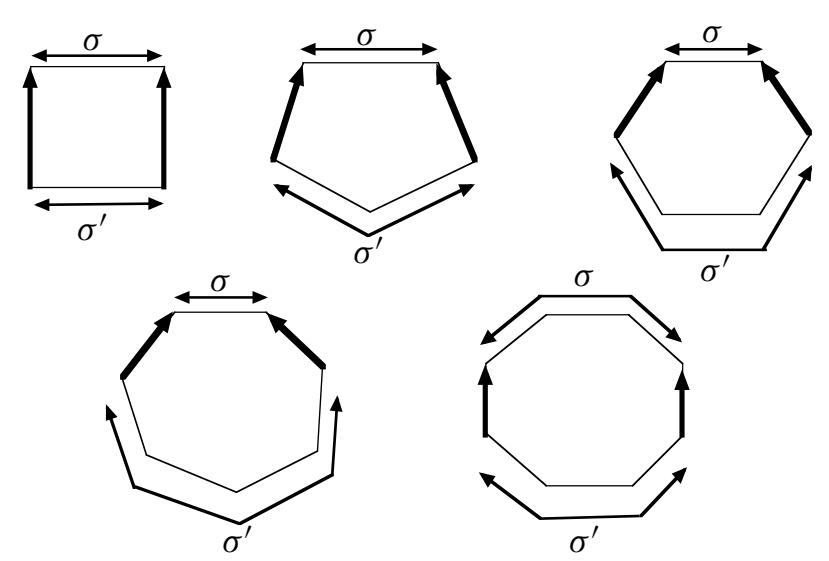

Figure 8: Even-parallel oriented edges

More generally if $k=4 f+r$ with $0 \leq r<4$ then $\ell=2 f-1$ and $\ell^{\prime}=2 f-1+r$.

Note that parallelism and even-parallelism inside a polygon coincide if the number of sides of this polygon is of the form $4 f$ or $4 f+1$. For example in a square complex $\|=\|_{e}$.

Definition 12.5 (e-walls) Let $X$ denote a polygonal complex. An $e$-wall of $X$ is an equivalence class of $\|_{e}$.

We say that an e-wall $M$ passes through an oriented edge $\vec{a}$ whenever $\vec{a} \in M$. We say that $a$ wall $M$ separates a polygon $\pi$ of $X$ whenever $M$ passes through oriented edges $\vec{a}, \vec{a}^{\prime}$ with $a \subset \partial \pi, a^{\prime} \subset \partial \pi$ and $\vec{a}, \vec{a}^{\prime}$ are even-parallel inside $\pi$.

An e-diameter of a polygon $\pi$ of $X$ is the union of two edges $[v, p],\left[v^{\prime}, p\right]$ of $X^{\prime}$ such that $p$ is the center of $\pi$, and there are distinct oriented edges $\vec{a}, \vec{a}^{\prime} \subset \partial \pi$ which are even-parallel in $\pi$, and whose centers are $v, v^{\prime}$. The e-diameter is then said to be dual to $\vec{a}, \vec{a}^{\prime}$.

The geometric e-wall associated to an e-wall $M$ is the subcomplex $|M|$ of the first barycentric subdivision $X^{\prime}$, union of those e-diameters of polygons which are dual to $\vec{a}, \vec{a}^{\prime}$, with $\vec{a}, \vec{a}^{\prime} \in M$.

If some $e$-wall $M$ passes through an oriented edge $\vec{a}$, we will also say that $M$ is dual to $\vec{a}$, and that $M$ is dual to $a$. For any $e$-wall $M$ we will denote by $V(M)$ the set of vertices $v$ belonging to an edge $a$ to which $M$ is dual. 
Note that a combinatorial map $f: X \rightarrow Y$ maps an $e$-wall of $X$ inside an $e$-wall of $Y$. The same is true for the associated geometric $e$-walls.

Changing walls to $e$-walls, the proof of the following lemma is exactly the same as that of Lemma 9.4.

Lemma 12.6 Assume that $X$ is locally compact, satisfies condition $\left(\mathrm{C}^{4}\right)$ and is simply connected. Equip $X$ with the $\left(\mathrm{C}^{4}\right)$-metric. Then:

(1) Each geometric $e-w a l l$ is a totally geodesic subtree.

(2) Each geometric $e$-wall separates $X$ into two connected components.

(3) Two opposite oriented edges are not even-parallel.

(4) The intersection of a geometric $e-w a l l$ and a polygon is either empty or a single diameter. In particular a geometric $e-w a l l|M|$ is the first barycentric subdivision of a tree whose vertices correspond to rank 1 vertices of $|M|$, and whose center of edges correspond to rank 2 vertices of $|M|$.

\section{Systems of local reflections}

Definition 13.1 (Edge neighbourhoods) Let $v$ denote some rank-1 vertex of $A$. Then $v$ is adjacent to exactly two vertices $x, y$ of rank 0 . The edge neighbourhood of $A$ with center $v$ is $U(v)=B(x) \cup B(y)$. Note that $U(v)$ is the first regular neighbourhood in $X$ of the edge with center $v$.

Let us define a weight $m: A^{\{2\}} \rightarrow\{2,3, \ldots\}$ by the rule $m(p)=m(e)$, where $e$ is the edge of $L=\partial B_{*}$ whose center is $\rho(p)$. In other words $m(p)$ is half the number of sides of the polygon of $X$ with center $p$. For any subcomplex $K \subset A$ we will denote by $\operatorname{Aut}_{\mathrm{rk}}(K, m)$ the group of automorphisms of $K$ preserving the rank and the weight $m$ on $K$. Clearly $\operatorname{Aut}_{\mathrm{rk}}(A, m)=\operatorname{Aut}_{\mathrm{rk}}(A)$.

The following notions were introduced in [20] for arbitrary polygonal complexes all of whose polygons have the same number of sides.

Definition 13.2 Let $v$ denote some rank-1 vertex of $A$; let $x, y$ denote its two adjacent rank-0 vertices. A local reflection at $v$ is an automorphism $\sigma$ of the edge neighbourhood $U(v)$ that exchanges $x$ and $y$, preserves the rank and the weight $m$, and fixes pointwise the facet of center $v$.

A system of local reflections on $A$ is a choice $\left(\sigma_{v}\right)_{v}$ for every rank-1 vertex $v$ of a local reflection $\sigma_{v}$ at $v$.

Algebraic 83 Geometric Topology, Volume 6 (2006) 
Let $\sigma$ denote some system of local reflections. Let $\varphi \in \operatorname{Aut}_{\mathrm{rk}}(A)$. For any vertex $v$ of rank 1 consider the local reflection at $v$ given by $\varphi \circ \sigma_{\varphi^{-1}(v)} \circ \varphi^{-1}$. This defines a new system of local reflections, denoted by $\varphi_{*}(\sigma)$. We thus obtain an action of $\operatorname{Aut}_{\mathrm{rk}}(A)$ on the set of systems of local reflections. Let $\operatorname{Aut}_{\mathrm{rk}}(A, \sigma)$ denote the stabilizer of $\sigma$.

For any triangle $\tau$ of $A$, let $\pi$ denote the polygon of $X$ whose first barycentric subdivision contains $\tau$. Let $\left(\overrightarrow{a_{1}}, \ldots, \overrightarrow{a_{2 m}}\right)$ denote the combinatorial edge-path of $X$ winding once around $\pi$, such that the first barycentric subdivision of $a_{1}$ contains an edge of $\tau$. Then the holonomy of a system of local reflections $\sigma$ at $\tau$ is the composition $h_{\sigma}(\tau)=\sigma_{v_{2 m}} \circ \cdots \circ \sigma_{v_{1}}$, where $v_{j}$ denotes the center of $a_{j}$. Note that $h_{\sigma}(\tau)$ is an automorphism of the block $B(\tau)$ containing $\tau$. It is straightforward that $h_{\sigma}(\tau)$ fixes the rank-2 vertex $p$ of $\tau$ ( $p$ is the center of the polygon $\pi$ ). Let $\tau^{\prime}$ be the other triangle of $B(\tau)$ containing $p$ : then $h_{\sigma}\left(\tau^{\prime}\right)=h_{\sigma}(\tau)^{-1}$. In fact $h_{\sigma}(\tau)$ fixes $\tau$ and $\tau^{\prime}$.

We say that a system $\sigma$ of local reflections has no holonomy if for every triangle $\tau$ we have $h_{\sigma}(\tau)=1$.

Example 13.3 For each rank-1 vertex $v=w v_{i}$ the stabilizer of $v$ in $W$ is the conjugate $w\left\{1, s_{i}\right\} w^{-1}$. Thus we may restrict the global reflection $w s_{i} w^{-1}$ to the edge neighbourhood $U(v)$. This yields a system of local reflections on $A$, which we will denote by $\sigma^{W}$.

We observe that $\sigma^{W}$ has no holonomy because $W$ acts freely on blocks.

Proposition 13.4 Let $\sigma, \sigma^{\prime}$ denote two systems of local reflections on $A$ with no holonomy. Let $B, B^{\prime}$ denote any two blocks of $A$. Let $f: B \rightarrow B^{\prime}$ denote any germ, that is, any isomorphism of blocks preserving the rank and preserving the weight $m$ on rank-2 vertices. Then $f$ extends to a unique automorphism $\bar{f} \in \operatorname{Aut}_{\mathrm{rk}}(A)$ such that $\bar{f}_{*}(\sigma)=\sigma^{\prime}$.

Proof The proof is exactly parallel to the proof of Proposition 6.5. We just give a skeleton, details are left to the reader.

Define a gallery to be a sequence of blocks such that two consecutive blocks are adjacent in the sense that they are equal or they share a facet.

Then to each pair $(f, G)$ where $f: B \rightarrow B^{\prime}$ is a germ and $G=\left(B_{0}=B, B_{1}, \ldots, B_{n}\right)$ a gallery, we associate a gallery $f(G)=\left(B_{0}^{\prime}=B^{\prime}, B_{1}^{\prime}, \ldots, B_{n}^{\prime}\right)$ and a sequence $G(f)=\left(f_{0}, f_{1}, \ldots, f_{n}\right)$ of germs $f_{i}: B_{i} \rightarrow B_{i}^{\prime}$ such that

(1) if $B_{i+1}=B_{i}$ then $B_{i+1}^{\prime}=B_{i}^{\prime}, f_{i+1}=f_{i}$

(2) if $B_{i+1} \cap B_{i}$ is a facet with center $v_{i}$ then $B_{i+1}^{\prime}$ is the block of $A$ whose intersection with $B_{i}^{\prime}$ is the facet of center $v_{i}^{\prime}=f_{i}\left(v_{i}\right)$, and $f_{i+1}^{\prime}=\sigma_{v_{i}^{\prime}}^{\prime} \circ f_{i} \circ \sigma_{v_{i}}$. 
If $f$ extends to an automorphism $\bar{f} \in \operatorname{Aut}_{\mathrm{rk}}(A)$ conjugating $\sigma$ with $\sigma^{\prime}$ then for each gallery $G$ we have $\bar{f}(G)=f(G)$ and $\omega(G(f))=\left.\bar{f}\right|_{\omega(G)}$ with $\omega(G)=B_{n}$ and $\omega(G(f))=f_{n}$. This proves uniqueness.

If $G$ is closed, then since $\sigma, \sigma^{\prime}$ have no holonomy the last germ of $G(f)$ is $f$ (it suffices to check this when $G$ is a gallery winding once around a polygon of $X$ ).

Thus we have $\omega(G(f))=\omega\left(G^{\prime}(f)\right)$, for any two galleries $G$ and $G^{\prime}$ from $B$ to some fixed block. This way we associate to each block a germ defined on this block: these germs fit together and define a combinatorial map $\bar{f}: A \rightarrow A$.

We then see that $\bar{f}$ is a rank-preserving automorphism conjugating $\sigma$ with $\sigma^{\prime}$.

Corollary 13.5 Let $\sigma$ denote some system of local reflections on $A$ with no holonomy. Then $\operatorname{Aut}_{\mathrm{rk}}(A, \sigma)$ is conjugate with $\operatorname{Aut}_{\mathrm{rk}}\left(A, \sigma^{W}\right)$ inside $\operatorname{Aut}_{\mathrm{rk}}(A)$.

The stabilizer of a block $B$ in $\operatorname{Aut}_{\mathrm{rk}}(A, \sigma)$ is isomorphic (by restriction) to the group of all germs $f: B \rightarrow B$. In particular $\operatorname{Aut}_{\mathrm{rk}}(A, \sigma)$ is a uniform lattice of $A$.

The group $\operatorname{Aut}_{\mathrm{rk}}\left(A, \sigma^{W}\right)$ is the semidirect product of $W$ and the finite group of those automorphisms of the graph $L$ which preserve the weight $m$ (this group acts naturally on $W$ ).

Proof The proof is similar to the proof of Corollary 6.7. The first assertion follows by Proposition 13.4 with $\sigma^{\prime}=\sigma^{W}$. For the second assertion use Proposition 13.4 with $\sigma^{\prime}=\sigma$.

The last affirmation is a consequence of the previous one, since $W$ is already simplytransitive on blocks and for any block $B$ we have $\operatorname{Aut}_{\mathrm{rk}}(B, m) \simeq \operatorname{Aut}(L, m)$.

Proof of Theorem 1.9 Assume first the uniform lattice $\Gamma$ of $X(m, L)$ is commensurable to $W(m, L)$ in Aut $X(m, L))$. Let $f$ denote an automorphism of $X(m, L)$ conjugating a finite index subgroup of $W$ onto a finite index subgroup $\Gamma^{\prime} \subset \Gamma$. Then $\Gamma^{\prime} \subset \operatorname{Aut}_{\mathrm{rk}}(A, \sigma)$ where $\sigma$ is the system of local reflections without holonomy obtained by conjugating $\sigma^{W}$ by $f$.

Conversely assume $\Gamma$ admits a finite index subgroup $\Gamma^{\prime}$ preserving a system of local reflections $\sigma$ whose holonomy is trivial. Then by Corollary 13.5, there exists an automorphism $f \in \operatorname{Aut}_{\mathrm{rk}}(A)$ which conjugates $\operatorname{Aut}_{\mathrm{rk}}(A, \sigma)$ with $\operatorname{Aut}_{\mathrm{rk}}\left(A, \sigma^{W}\right)$, and furthermore $W$ is of finite index in $\operatorname{Aut}_{\mathrm{rk}}\left(A, \sigma^{W}\right)$. It follows that $f$ conjugates a finite index subgroup of $\Gamma^{\prime}$ onto a finite index subgroup of $W$. Hence $f$ commensurates $\Gamma$ with $W$. 


\section{Killing the holonomy}

Lemma 14.1 Let $\Gamma^{\prime} \subset \operatorname{Aut}_{\mathrm{rk}}(A)$ denote any subgroup acting freely on rank-1 vertices. Then $\Gamma^{\prime}$ preserves some system of local reflections.

Proof Let $\left(v_{t}\right)_{t \in T}$ denote a set of representatives of rank-1 vertices under the action of $\Gamma^{\prime}$. Choose arbitrarily a local reflection $\sigma_{v_{t}}$ at each $v_{t}$, for example use $\sigma^{W}$. Then extend $\sigma$ using conjugation by elements of $\Gamma^{\prime}$.

\subsection{Modification of a system of local reflections}

Definition 14.2 (Fields) For each triangle $\tau$ of $A$ we denote by $B(\tau)$ the block containing $\tau$, by $\phi^{+}(\tau)$ the facet of $B$ meeting $\tau$ along an edge, and by $F^{+}(\tau)$ the subgroup of $\operatorname{Aut}_{\mathrm{rk}}(B(\tau), m)$ fixing $\phi^{+}(\tau)$ pointwise. We also denote by $\phi^{-}(\tau)$ the other facet of $B(\tau)$ meeting $\tau$ (at the vertex of rank 2). We introduce the subgroup $F^{-}(\tau)$ of $\operatorname{Aut}_{\mathrm{rk}}(B(\tau), m)$ fixing $\phi^{-}(\tau)$ pointwise.

A semi-edge of $X$ is an edge $e$ of $A$ whose vertices have rank 0 and 1 . Given a semi-edge $e$, we will always denote by $\bar{e}$ the unique other semi-edge of $X$ meeting $e$. Note that the union of $e$ and $\bar{e}$ is the barycentric subdivision of an edge of $X$.

For each semi-edge $e$ of $X$ we denote by $B(e)$ the block containing $e$, by $\phi(e)$ the facet of $B$ meeting $e$, and by $F(e)$ the subgroup of $\operatorname{Aut}_{\mathrm{rk}}(B(e), m)$ fixing $\phi(e)$ pointwise.

A field of rank 2 on $A$ is a map $f$ assigning to each triangle $\tau$ of $A$ an element $f(\tau)$ of $F^{+}(\tau)$. Similarly a map $f$ assigning to each semi-edge $e$ of $X$ an element $f(e)$ of $F(e)$ is a field of rank 1 on $A$.

The set of fields of given rank has a natural structure of group: for example in rank 1 we set $f_{1} f_{2}(e)=f_{1}(e) f_{2}(e)$.

The support of a rank-1 field $f$ is the set $\operatorname{Supp}(f)$ of semi-edges $e$ of $X$ such that $f(e) \neq 1$. Similarly the of a rank-2 field $\varphi$ is the set $\operatorname{Supp}(\varphi)$ of triangles $\tau$ of $A$ such that $\varphi(\tau) \neq 1$.

The group $\operatorname{Aut}_{\mathrm{rk}}(A)$ acts on fields by conjugation: for $\gamma \in \operatorname{Aut}_{\mathrm{rk}}(A)$ and $f$ a field we define $\gamma f$ by $\gamma f(x)=\gamma \circ f\left(\gamma^{-1} x\right) \circ \gamma^{-1}$.

Definition 14.3 Let $\sigma$ denote a system of local reflections. Let $f$ denote some field of rank 1 . We say that $f$ is symmetric with respect to $\sigma$ (or $\sigma$-symmetric for short) whenever for every rank-1 vertex $v$ and for every semi-edge $e$ of $X$ containing $v$, we have $\left.\sigma_{v}\right|_{B(e)} \circ f(e)=\left.f(\bar{e})^{-1} \circ \sigma_{v}\right|_{B(e)}$. 
Assume $f$ is $\sigma$-symmetric. Consider some rank-1 vertex $v$; let $e_{1}, e_{2}$ be the two semi-edges of $X$ containing $v$. We define an automorphism $\sigma_{v}^{\prime}$ of $U(v)$ as follows:

(1) for $p \in B\left(e_{1}\right)$ we set $\sigma_{v}^{\prime}(p)=\sigma_{v}\left(f\left(e_{1}\right) p\right)$

(2) for $p \in B\left(e_{2}\right)$ we set $\sigma_{v}^{\prime}(p)=\sigma_{v}\left(f\left(e_{2}\right) p\right)$.

This is well-defined since $B\left(e_{1}\right) \cap B\left(e_{2}\right)=\phi(v)$ and $f\left(e_{i}\right), \sigma_{v}$ fix $\phi(v)$ pointwise. Clearly $\sigma_{v}^{\prime}$ exchanges $e_{1}$ and $e_{2}$. Furthermore using invariance, we have for $p \in B\left(e_{1}\right)$

$$
\sigma_{v}^{\prime 2}(p)=\sigma_{v}\left(f\left(e_{2}\right) \sigma_{v}\left(f\left(e_{1}\right) p\right)\right)=\sigma_{v}\left(f\left(e_{2}\right) f\left(e_{2}\right)^{-1} \sigma_{v}(p)\right)=p .
$$

Thus $\sigma_{v}^{\prime}$ is an involution. We have thus obtained a new system of local reflections, which we will denote by $\sigma f$.

Remark 14.4 For any two systems of local reflections $\sigma, \sigma^{\prime}$ there exists one and only one $\sigma$-symmetric rank-1 field $f$ such that $\sigma^{\prime}=\sigma f$. This field is defined by $f(e) p=\sigma_{v}{ }^{-1}\left(\sigma_{v}^{\prime}(p)\right)$ where $v$ is the rank 0 vertex of the semi-edge $e$.

We are going to modify systems of local reflections by applying convenient symmetric rank-1 field, in such a way that the holonomy of the resulting system of local reflections is smaller.

Lemma 14.5 (Holonomy of $\sigma f$ ) Let $\sigma$ denote some system of local reflections. Let $f$ denote some $\sigma$-symmetric rank-1 field. Let $\tau$ denote some triangle of $A$. We introduce a sequence $\underline{g}=\underline{g}(\tau, \sigma, f)=\left(g_{0}, \ldots, g_{2 m}\right)$ of automorphisms of $B(\tau)$ defined as follows.

Let $\pi$ denote the polygon of $X$ containing $\tau$, let $\left(\overrightarrow{a_{1}}, \ldots, \overrightarrow{a_{2 m}}\right)$ denote the combinatorial edge-path of $X$ winding once around $\pi$ such that the first barycentric subdivision of $a_{1}$ contains an edge of $\tau$ (see Definition 13.2), and let $v_{i}$ denote the rank 1 vertex of $A$ at the center of $a_{i}$. Let $e_{i}$ denote the initial semi-edge of $\overrightarrow{a_{i}}$. We set

$g_{i}=g_{i}(\tau, \sigma, f)=\left(\left.\left.\sigma_{v_{1}}\right|_{B\left(e_{2}\right)} \circ \cdots \circ \sigma_{v_{i-1}}\right|_{B\left(e_{i}\right)}\right) \circ f\left(e_{i}\right) \circ\left(\left.\left.\sigma_{v_{i-1}}\right|_{B\left(e_{i-1}\right)} \circ \cdots \circ \sigma_{v_{1}}\right|_{B\left(e_{1}\right)}\right)$.

Then $g_{i}=f\left(e_{1}\right) \in F^{+}(\tau)$ for $i$ odd, $g_{i} \in F^{-}(\tau)$ for $i$ even, and furthermore

$$
h_{\sigma f}(\tau)=h_{\sigma}(\tau) \circ g_{2 m} \circ \cdots \circ g_{1},
$$

where $2 m$ denotes the number of vertices of the polygon $\pi$.

Proof By definition we have

$h_{\sigma f}(\tau)=(\sigma f)_{v_{2 m}} \circ \cdots \circ(\sigma f)_{v_{1}}=\sigma_{v_{2 m}} \circ f\left(e_{2 m}\right) \circ \sigma_{v_{2 m-1}} \circ f\left(e_{2 m-1}\right) \circ \cdots \circ \sigma_{v_{1}} \circ f\left(e_{1}\right)$. 
We also have

$$
\sigma_{v_{2 m}} \circ f\left(e_{2 m}\right) \circ \sigma_{v_{2 m-1}} \circ f\left(e_{2 m-1}\right)=\sigma_{v_{2 m}} \circ \sigma_{v_{2 m-1}} \circ f\left(e_{2 m}\right)^{\sigma_{v_{2 m-1}} \circ f\left(e_{2 m-1}\right) .}
$$

If we make successively each $\sigma_{v_{i}}$ go from the right to the left we get the desired formula $h_{\sigma f}(\tau)=h_{\sigma}(\tau) \circ g_{2 m} \circ \cdots \circ g_{1}$.

Now local reflections preserve rank and weight. So conjugation by the inverse of the composition $t_{i}=\left.\left.\sigma_{v_{1}}\right|_{B\left(e_{2}\right)} \circ \cdots \circ \sigma_{v_{i-1}}\right|_{B\left(e_{i}\right)}$ sends $F\left(e_{i}\right)$ onto $F\left(t_{i}\left(e_{i}\right)\right) \subset F(\tau)$. When $i$ is odd, $F\left(t_{i}\left(e_{i}\right)\right)=F^{+}(\tau)$, and $F\left(t_{i}\left(e_{i}\right)\right)=F^{-}(\tau)$ otherwise.

The following result already appears in [20].

Corollary 14.6 For every fixed triangle $\tau$, the set of all possible holonomies $h_{\sigma}(\tau)$ (as $\sigma$ varies in the set of systems of local reflections) is the whole product subgroup $F^{+}(\tau) F^{-}(\tau)=F^{-}(\tau) F^{+}(\tau)$.

Proof By Remark 14.4, any system of local reflection $\sigma$ is of the form $\sigma^{W} f$, for $f$ some $\sigma^{W}$-symmetric rank-1 field. Thus by Lemma 14.5, the set of all possible holonomies $h_{\sigma}(\tau)$ is the set of all possible products $g_{2 m} \circ \cdots \circ g_{1}$.

Note that the subgroups $F^{+}(\tau)$ and $F^{-}(\tau)$ normalize each other. Thus the group they generate consists in elements of the form $g^{+} g^{-}$with $g^{+} \in F^{+}(\tau)$ and $g^{-} \in F^{-}(\tau)$. So we always have $h_{\sigma}(\tau) \in F^{+}(\tau) F^{-}(\tau)$.

Conversely choose a $\sigma^{W}$-symmetric rank-1 field which takes an arbitrary value on $e_{1}$ and $e_{2}$, and is trivial outside the edges $e_{1}, e_{2}, \bar{e}_{1}$ and $\bar{e}_{2}$. Then we see that $h_{\sigma}(\tau)$ is any element of $F^{-}(\tau) F^{+}(\tau)=F^{+}(\tau) F^{-}(\tau)$.

\subsection{Groups acting cleanly}

Definition 14.7 (Self-intersecting $e$-wall) Let $X$ be some simply connected polygonal complex and let $\Gamma$ denote some subgroup of $\operatorname{Aut}(X)$. We say that an $e$-wall $M$ is self-intersecting under $\Gamma$ whenever there exists $\gamma \in \Gamma$ such that $M$ and $\gamma M$ are distinct, but the geometric $e$-walls associated to $M$ and $\gamma M$ have nonempty intersection. We say that $\Gamma$ acts without e-self-intersection whenever no $e$-wall is self-intersecting under $\Gamma$.

We say that $\Gamma \subset \operatorname{Aut}(X)$ is clean whenever it acts without e-self-intersection and moreover it acts freely on $X$. 
Lemma 14.8 Let $\Gamma$ denote a discrete cocompact group of automorphism of some simply connected polygonal complex $X$. Assume that the stabilizers of $e-$ walls are separable in $\Gamma$.

Then there is a finite index subgroup $\Gamma^{\prime} \subset \Gamma$ acting without e-self-intersection.

The proof is the same as that of Lemma 10.2; details are left to the reader.

Corollary 14.9 Assume $(W, S)$ is two-dimensional. Let $\Gamma$ denote a residually finite uniform lattice of $\operatorname{Aut}_{\mathrm{rk}}(A)=\operatorname{Aut}(X)$ all of whose $e-$ wall stabilizers are separable. Then there is a finite index subgroup $\Gamma^{\prime} \subset \Gamma$ such that $\Gamma^{\prime}$ is clean.

Proof By Lemma 14.8, it suffices to prove that $\Gamma$ virtually acts freely on $A$.

Since $A$ admits a CAT(0) length metric by Lemma 11.4, any finite order element of $\Gamma$ has a fixed point in $A$. Thus, up to conjugation, there are finitely many finite order elements in $\Gamma$. Since $\Gamma$ is residually finite there is a finite index torsion free subgroup $\Gamma^{\prime} \subset \Gamma$. Since $\Gamma$ acts discretely we see that $\Gamma^{\prime}$ has to act freely.

In fact the separability of $e$-wall stabilizers imply the residual finiteness of $\Gamma$.

Remark 14.10 Assume $A$ is two-dimensional and all $m_{i j}$ are even, for example, $W$ is right-angled. Then $e$-walls are walls, and geometric walls are the fixed point sets of reflections of $W$. Thus an $e$-wall stabilizer is the centralizer of a reflection. Since Coxeter groups are residually finite the centralizers of their involutions are separable. So in this case Corollary 14.9 applies: $W$ has a clean finite index subgroup.

In [25] we prove that in every Gromov-hyperbolic Coxeter group, each quasiconvex subgroup is separable. It follows that in this case also $W$ has a clean finite index subgroup.

The general two-dimensional case remains open.

\subsection{Killing half of the holonomy along an $e$-wall}

Until the end of this subsection we assume that $W$ is two-dimensional, and we consider a fixed clean uniform lattice $\Gamma^{0}$ of $X$.

Definition 14.11 $((i, j)$-adjacency) Let $i$ and $j$ denote two distinct integers of $\{0,1,2\}$. We say that two triangles $\tau_{1}$ and $\tau_{2}$ are $(i, j)$-adjacent whenever they share an edge whose endpoints are vertices $x$ and $y$ of ranks $i$ and $j$, respectively. We say that two triangles are adjacent if they are $(i, j)$-adjacent for some pair $(i, j)$.

Algebraic ${ }^{3} \mathcal{G}$ Geometric Topology, Volume 6 (2006) 
The $(i, j)$-adjacency condition is an equivalence relation on the set of triangles. Every $(0,2)$-adjacency class contains two triangles. For each triangle $\tau$ we will denote by $\tau^{\prime}$ the second triangle of the $(0,2)$-adjacency class of $\tau$. Note that $\tau$ and $\tau^{\prime}$ are contained in the same polygon.

Definition 14.12 Since $\Gamma^{0}$ preserves rank and acts freely on rank-2 vertices, it acts freely on the set of polygons of $X$, and we may find a transversal set $\mathcal{T}$ of triangles with the following properties:

(1) $\mathcal{T}$ is $\Gamma^{0}$-invariant

(2) for each polygon $\pi$, the set of triangles of $\mathcal{T}$ contained in $\pi$ consists of a $(0,2)$-adjacency class denoted by $\mathcal{T}_{\pi}$.

From now on we choose such a set $\mathcal{T}$. We will use it to compute the holonomy of various systems of local reflections.

Let $\pi$ denote a polygon of $X$. Since $\partial \pi$ has an even number of edges there is a labeling of these edges in $\{+,-\}$ such that two adjacent edges receive distinct labels. This labeling defines a labeling on the set of rank-1 vertices of $\pi$. For any $e$-wall $M$ separating the polygon $\pi$ the two edges of $\partial \pi$ dual to $M$ receive the same label, say $\varepsilon$. Then the rank-1 vertex of exactly one of the triangles of $\mathcal{T}_{\pi}$ has the same label $\varepsilon$ : we will denote this triangle by $\tau(\pi, M)$. Note that $\mathcal{T}_{\pi}=\left\{\tau(\pi, M), \tau(\pi, M)^{\prime}\right\}$.

Observe that parallel oriented edges inside $\pi$ receive the same label if and only if they are also even-parallel. This is why we need the notion of $e$-walls, justifying our terminology of even-parallelism.

Definition 14.13 Let $\sigma$ denote any system of local reflections. A decomposition of the holonomy $h_{\sigma}$ is a rank-2 field $\varphi$ with support contained in $\mathcal{T}$ such that for any triangle $\tau \in \mathcal{T}$ we have $h_{\sigma}(\tau)=\varphi\left(\tau^{\prime}\right)^{-1} \varphi(\tau)$.

Remark 14.14 Let $\sigma$ denote any system of local reflections. Then $\sigma$ always admits a decomposition $\varphi$ by Corollary 14.6. Furthermore if $\sigma$ is invariant under a subgroup $\Gamma \subset \operatorname{Aut}_{\mathrm{rk}}(A)$ acting freely on vertices then the decomposition $\varphi$ of $\sigma$ may be chosen to be $\Gamma$-invariant.

Theorem 14.15 Assume $(W, S)$ is two-dimensional and $I$ does not contain any subset $\{i, j, \ell\}$ with $m_{i j} \leq 3, m_{j \ell}=3, m_{\ell i}<+\infty$ (so that $X$ satisfies condition $\left(\mathrm{C}^{4}\right)$ by Lemma 11.4).

Let $\Gamma^{0}$ denote a clean uniform lattice in $\operatorname{Aut}_{\mathrm{rk}}(A)$. where each finite index subgroup of a wall-stabilizer in $\Gamma^{0}$ contains the intersection with the wall-stabilizer of a finite 
index subgroup in $\Gamma^{0}$. Consider such a finite index normal subgroup $\Gamma \subset \Gamma^{0}$, and let $\sigma$ denote a $\Gamma$-invariant system of local reflections and $\varphi$ denote a rank-2 field invariant under $\Gamma$ which is a decomposition of $h_{\sigma}$.

For any $e-w a l l M$ there is a system of local reflections $\sigma^{\prime}$, a rank-2 field $\varphi^{\prime}$ and a finite index normal subgroup $\Gamma^{\prime} \subset \Gamma^{0}$ such that:

(1) $\sigma^{\prime}$ and $\varphi^{\prime}$ are invariant under $\Gamma^{\prime}$

(2) $\varphi^{\prime}$ is a decomposition of $h_{\sigma^{\prime}}$

(3) $\varphi^{\prime}(\tau)=\varphi(\tau)$ on triangles $\tau \in \mathcal{T}_{\pi}$ if a polygon $\pi$ is not separated by any translate $\gamma M$ for $\gamma \in \Gamma^{0}$

(4) $\varphi^{\prime}(\tau(\pi, \gamma M))=1$ and $\varphi^{\prime}\left(\tau(\pi, \gamma M)^{\prime}\right)=\varphi\left(\tau(\pi, \gamma M)^{\prime}\right)$ if a polygon $\pi$ is separated by some (necessarily unique) translate $\gamma M$ for $\gamma \in \Gamma^{0}$.

The theorem says that up to passing to a finite index subgroup $\Gamma^{\prime}$ we may simplify the decomposition of the holonomy of an invariant system of local reflections along $\Gamma^{0}$-translates of the wall $M$.

Proof We first study the set $F=F(\sigma, \varphi, M)$ of rank-1 fields $f$ with the following properties:

(1) $f$ is $\sigma$-symmetric

(2) any semi-edge of $\operatorname{Supp}(f)$ is contained in an edge dual to $M$

(3) for any polygon $\pi$ separated by $M$, using the notation of Lemma 14.5 we have

$$
g_{i_{2}(\pi, M)}(\tau(\pi, M), \sigma, f) g_{i_{1}(\pi, M)}(\tau(\pi, M), \sigma, f)=\varphi(\tau(\pi, M))^{-1} .
$$

We must explain the definition of the integers $i_{1}(\pi, M)$ and $i_{2}(\pi, M)$. The triangle $\tau(\pi, M)$ defines a sequence of semi-edges $\left(e_{1}, \ldots, e_{2 m_{\pi}}\right)$ winding once around $\pi$ with $e_{1} \subset \tau(\pi, M)$, as in Lemma 14.5. Then $i_{1}(\pi, M)$ is the first integer $1 \leq i \leq 2 m_{\pi}$ such that $e_{i}$ meets $|M|$ and $i_{2}(\pi, M)$ is the second such integer. Note that $|M|$ meets only two of the semi-edges $e_{i}$.

If $m_{\pi}$ is even then $i_{2}(\pi, M)=i_{1}(\pi, M)+m_{\pi}$, and in contrast if $m_{\pi}$ is odd then $i_{2}(\pi, M)=i_{1}(\pi, M)+m_{\pi}-1$.

Lemma 14.16 The set $F$ is finite, nonempty and invariant under the stabilizer $\Gamma_{M}$ of $M$ in $\Gamma$. 
Proof To prove the two first assertions we consider a rank-1 vertex $v$ in $|M|$ together with a semi-edge $e$ of $X$ containing $v$, and we show that the map $F \rightarrow F(e)$ sending $f \in F$ to $f(e) \in F(e)$ is a bijection.

Consider a combinatorial injective path $c=\left(v_{0}=v, v_{1}, \ldots, v_{n}=w\right)$ of the tree $T(M)$ whose first barycentric subdivision is $|M|$ (see Lemma 11.4 and Lemma 12.6). Any edge $\left(v_{i-1}, v_{i}\right)$ of this path is contained in a single polygon $\pi_{i}$ of $X$. With the above definition (using notation of Lemma 14.5) the semi-edges of the sequence $\left(e_{1}, \ldots, e_{2 m_{\pi}}\right)$ winding once around $\pi_{i}$ with $e_{1} \subset \tau\left(\pi_{i}, M\right)$ meet $|M|$ exactly at the indices $i_{1}:=i_{1}\left(\pi_{i}, M\right)$ and $i_{2}:=i_{2}\left(\pi_{i}, M\right)$. So if $w_{j}$ is the rank-1 vertex of $e_{j}$ we have $\left\{w_{i_{1}\left(\pi_{i}, M\right)}, w_{i_{2}\left(\pi_{i}, M\right)}\right\}=\left\{v_{i-1}, v_{i}\right\}$. To simplify notation we set $\tau\left(\pi_{i}, M\right)=\tau_{i}$. We also set $a_{i}=e_{i_{1}}$ and $\bar{a}_{i}=e_{i_{2}}$ if $v_{i-1} \in e_{i_{1}}$, and otherwise we set $a_{i}=e_{i_{2}}$ and $\bar{a}_{i}=e_{i_{1}}$.

As in Lemma 14.5, we define two isomorphisms from the block $B\left(\tau_{i}\right)$ onto $B\left(e_{i_{1}}\right)$ and from the block $B\left(\tau_{i}\right)$ onto $B\left(e_{i_{2}}\right)$, by composing local reflections. More precisely we define $s_{i, 1}=\left.\left.\sigma_{w_{i_{1}-1}}\right|_{B\left(e_{i_{1}-1}\right)} \circ \cdots \circ \sigma_{w_{1}}\right|_{B\left(e_{1}\right)}$ which is the identity if $i_{1}=1$, and $s_{i, 2}=\left.\left.\sigma_{w_{i_{2}-1}}\right|_{B\left(e_{i_{2}-1}\right)} \circ \cdots \circ \sigma_{w_{1}}\right|_{B\left(e_{1}\right)}$.

We claim that for any element $f$ of $F(e)$ and any semi-edge $e^{\prime}$ of $X$ containing the endpoint $w$ of $c$, if we set $\bar{a}_{0}=e$ and $a_{n+1}=e^{\prime}$, then there exists one and only one sequence $\left(\bar{f}_{0}, f_{1}, \bar{f}_{1}, f_{2}, \ldots, f_{n}, \bar{f}_{n}, f_{n+1}\right)$ such that

(1) $\bar{f}_{0}=f$; for $1 \leq i \leq n, f_{i} \in F\left(a_{i}\right), \bar{f}_{i} \in F\left(\bar{a}_{i}\right) ; f_{n+1} \in F\left(e^{\prime}\right)$

(2) for $0 \leq i \leq n$, if $a_{i+1}=\bar{a}_{i}$ then $f_{i+1}=\bar{f}_{i}$ and otherwise $f_{i+1}=\sigma_{v_{i}} \circ \bar{f}_{i} \circ \sigma_{v_{i}}$

(3) for $1 \leq i \leq n$, if $a_{i}=e_{i_{1}}$ then $\left(s_{i, 2}{ }^{-1} \circ \bar{f}_{i} \circ s_{i, 2}\right) \circ\left(s_{i, 1}{ }^{-1} \circ f_{i} \circ s_{i, 1}\right)=\varphi\left(\tau_{i}\right)^{-1}$ and otherwise $\left(s_{i, 2}{ }^{-1} \circ f_{i} \circ s_{i, 2}\right) \circ\left(s_{i, 1}{ }^{-1} \circ \bar{f}_{i} \circ s_{i, 1}\right)=\varphi\left(\tau_{i}\right)^{-1}$.

Given $f_{i} \in F\left(a_{i}\right)$ the last equation is always solvable in the unknown $\bar{f}_{i} \in F\left(\bar{a}_{i}\right)$ because the rank-1 vertices of $e_{1}, e_{i_{1}}, e_{i_{2}}$ are mutually at even distance on $\partial \pi_{i}$, so we see that the isomorphism $s_{i, 1}$ conjugates $F\left(e_{1}\right)=F^{+}\left(\tau_{i}\right)$ with $F\left(a_{i}\right)$ or $F\left(\bar{a}_{i}\right)$ (and similarly $s_{i, 2}$ conjugates $F^{+}\left(\tau_{i}\right)$ with $F\left(\bar{a}_{i}\right)$ or $F\left(a_{i}\right)$ ). The other conditions are trivial to satisfy. So the claim follows by induction on the length $n$ of the path $c$.

Let $e^{\prime}$ be a semi-edge $e^{\prime}$ of $X$ containing a rank-1 vertex $w$ of $|M|$, and denote by $c=\left(v_{0}=v, v_{1}, \ldots, v_{n}=w\right)$ the unique injective combinatorial path in the tree $T(M)$ from $v$ to $w$. Using the notation above, we define $f\left(e^{\prime}\right)$ by setting $f\left(e^{\prime}\right)=f_{n+1}$. For any semi-edge $e^{\prime \prime}$ not adjacent to $|M|$ we set $f\left(e^{\prime \prime}\right)=1$. We thus obtain the unique preimage of $f \in F(e)$ in $F$ under the restriction map $F \rightarrow F(e)$.

It remains to check that $F$ is invariant under $\Gamma_{M}$. For $f \in F$ and $\gamma \in \Gamma_{M}$ clearly $\gamma f$ is trivial outside the set of semi-edges adjacent to $|M|$. Also $\gamma f$ is $\sigma$-symmetric, 


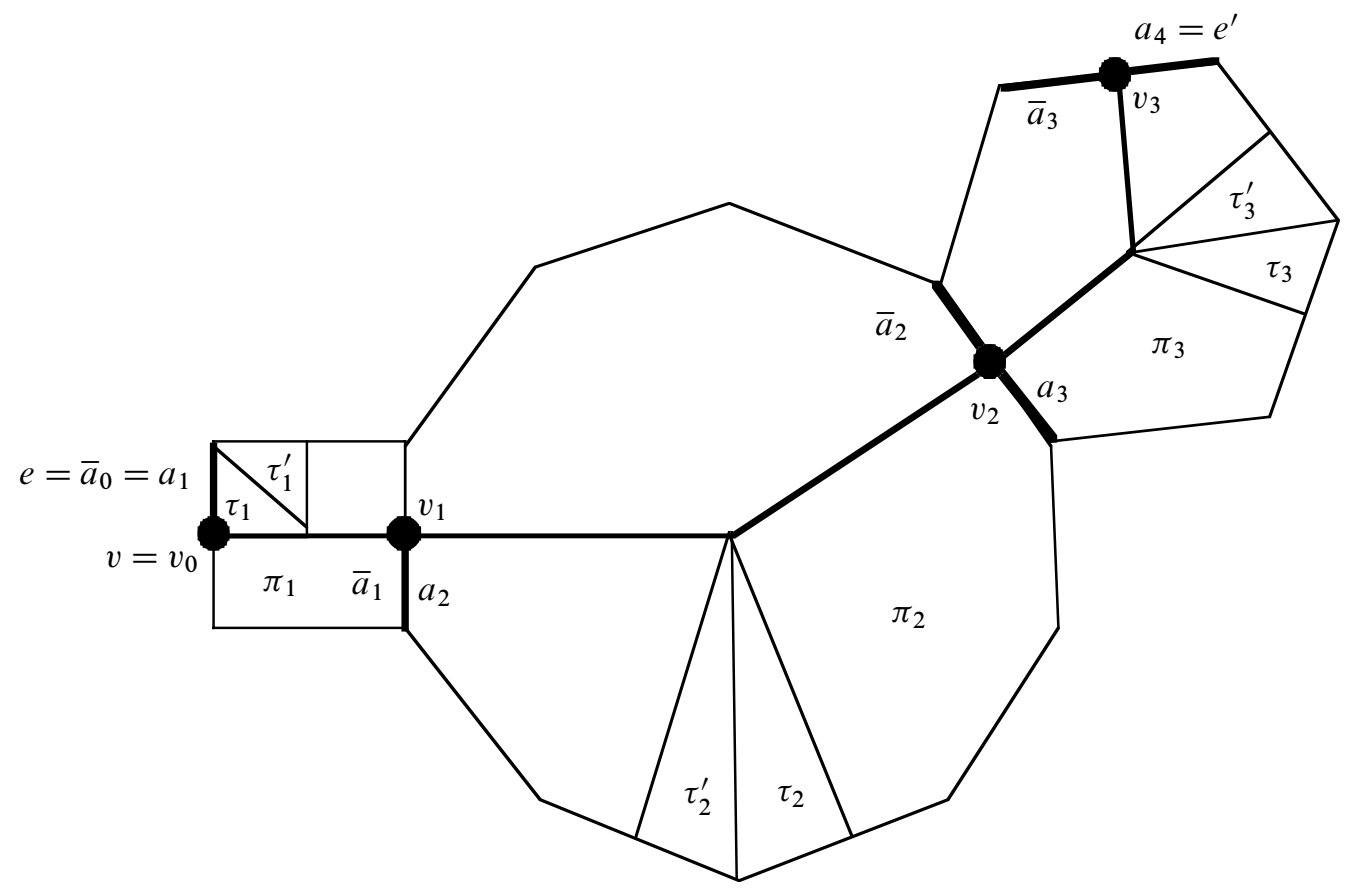

Figure 9: Extension of a rank-1 field along an $e$-wall

because $\sigma$ is invariant under $\Gamma$. The third relation defining elements of $F$ is still satisfied by $\gamma f$ because $\varphi$ is $\Gamma$-invariant and $g_{i}(\gamma \tau, \gamma \sigma, \gamma f)$ is the conjugate by $\gamma$ of $g_{i}(\tau, \sigma, f)$.

We construct $\sigma^{\prime}, \varphi^{\prime}$ and $\Gamma^{\prime}$ using the action of $\Gamma_{M}$ on the sets $F(c, M)$.

Since $F(\sigma, \varphi, M)$ is finite and $\Gamma_{M}$ acts on it, some finite index subgroup of $\Gamma_{M}$ fixes $F(\sigma, \varphi, M)$ pointwise. By the hypotheses, there exists a finite index subgroup $\Gamma^{\prime}(\sigma, \varphi) \subset \Gamma^{0}$ such that $\Gamma^{\prime}(\sigma, \varphi)_{M}$ acts trivially on $F$. We may even assume that $\Gamma^{\prime}(\sigma, \varphi)$ is normal in $\Gamma^{0}$ and contained in $\Gamma$.

Consider all pairs $(\rho, \psi)$ with a system $\rho$ of local reflections and a rank-2 field $\psi$ decomposing the holonomy $h_{\rho}$ where $\rho$ and $\psi$ are invariant under $\Gamma$. Observe that there are finitely many such pairs, because of $\Gamma$-invariance ( $\Gamma$ is cocompact). For each pair we consider a finite index normal subgroup $\Gamma^{\prime}(\rho, \psi) \subset \Gamma^{0}$ with $\Gamma^{\prime}(\rho, \psi) \subset \Gamma$ such that the stabilizer of $M$ in $\Gamma^{\prime}(\rho, \psi)$ acts trivially on $F(\rho, \psi, M)$. Then the intersection of all such $\Gamma^{\prime}(\rho, \psi)$ is a finite index normal subgroup $\Gamma^{\prime}$ of $\Gamma^{0}$ where $\Gamma^{\prime} \subset \Gamma$ and $\Gamma^{\prime} M$ acts trivially on all the sets $F(\rho, \psi, M)$. 
Choose elements $\gamma_{1}=1, \gamma_{2}, \ldots, \gamma_{k} \in \Gamma^{0}$ such that any $e$-wall $\gamma M$ with $\gamma \in \Gamma^{0}$ is equivalent modulo $\Gamma^{\prime}$ to a unique $\gamma_{i} M=M_{i}$. We now define a $\Gamma^{\prime}$-invariant rank-1 field $f$.

Pick elements $f_{i} \in F\left(\sigma, \varphi, M_{i}\right)$. We first check that $f_{i}$ is invariant under $\Gamma^{\prime} M_{i}$. Conjugation by $\gamma_{i}$ sends $F\left(\gamma_{i}^{-1} \sigma, \gamma_{i}^{-1} \varphi, M\right)$ to $F\left(\sigma, \varphi, \gamma_{i} M\right)$. Observe that $\gamma_{i}^{-1} \sigma$ and $\gamma_{i}^{-1} \varphi$ are invariant under $\Gamma$ (because $\Gamma$ is normal in $\Gamma^{0}$ ). So by construction $\Gamma^{\prime}{ }_{M}$ fixes $F\left(\gamma_{i}{ }^{-1} \sigma, \gamma_{i}{ }^{-1} \varphi, M\right)$. Thus $\gamma_{i} \Gamma^{\prime}{ }_{M} \gamma_{i}{ }^{-1}$ fixes $F\left(\sigma, \varphi, \gamma_{i} M\right)$. But $\Gamma^{\prime}$ is normal in $\Gamma^{0}$, so $\gamma_{i} \Gamma_{M}^{\prime} \gamma_{i}^{-1}=\Gamma^{\prime} M_{i}$.

For every translate $\gamma M, \gamma \in \Gamma^{0}$, we now choose some $\gamma^{\prime} \in \Gamma^{\prime}$ such that $\gamma^{\prime} \gamma_{i} M=\gamma M$ with $\gamma^{\prime}=1$ if $\gamma M$ is $\gamma_{i} M$ for some $i$.

For a semi-edge $e$ not adjacent to a geometric $e$-wall of the form $\gamma|M|$ with $\gamma \in \Gamma^{0}$, we set $f(e)=1$. For a semi-edge $e$ adjacent to $\gamma_{i}|M|$ we set $f(e)=f_{i}(e)$. Observe that by cleanliness $e$ is not adjacent to an other $\gamma_{j}|M|$.

More generally if the semi-edge $e$ is adjacent to a geometric $e$-wall of the form $\gamma|M|$ with $\gamma \in \Gamma^{0}$ and $\gamma M=\gamma^{\prime} \gamma_{i} M$, we set $f(e)=\gamma^{\prime} \circ f_{i}\left(\gamma^{\prime-1} e\right) \circ \gamma^{\prime-1}$. Again, by cleanliness $e$ is not adjacent to $\gamma^{\prime \prime}|M|$ with $\gamma M \neq \gamma^{\prime \prime} M$.

The rank-1 field $f$ is $\Gamma^{\prime}$-invariant because each $\Gamma^{\prime}{ }_{M_{i}}$ fixes $f_{i}$. Since $\Gamma^{\prime} \subset \Gamma$ each of the chosen elements $\gamma^{\prime}$ preserves $\sigma$. Then by construction $f$ is $\sigma$-symmetric. We set $\sigma^{\prime}=\sigma f$. This new system $\sigma^{\prime}$ of local reflections is also $\Gamma^{\prime}$-invariant.

We now compute the holonomy of $\sigma^{\prime}$ at some triangle $\tau \in \mathcal{T}$ and show how to decompose it. Let us denote by $\pi$ the polygon of $X$ containing $\tau$. We have either $\tau(\pi, M)=\tau$ or $\tau(\pi, M)=\tau^{\prime}$.

If $\pi$ is not separated by an $e$-wall of the form $\gamma M, \gamma \in \Gamma^{0}$, then for each semi-edge $e \subset \partial \pi$ we have $f(e)=1$. We then have $h_{\sigma^{\prime}}(\tau)=h_{\sigma}(\tau)=\varphi\left(\tau^{\prime}\right)^{-1} \varphi(\tau)$, by Lemma 14.5. We thus set $\varphi^{\prime}(\tau)=\varphi(\tau)$ and $\varphi^{\prime}\left(\tau^{\prime}\right)=\varphi\left(\tau^{\prime}\right)$.

Assume now that $\pi$ is separated by at least one $\Gamma$-translate of $M$. Since cleanliness implies that distinct $\Gamma$-translates of $M$ have disjoint associated geometric $e$-walls, there exists a unique translate $\gamma M$ which separates $\pi$. By Lemma 14.5, we have $h_{\sigma^{\prime}}(\tau(\pi, \gamma M))=h_{\sigma}(\tau(\pi, \gamma M)) \circ g_{2 m_{\pi}} \circ \cdots \circ g_{1}$. On the set of semi-edges adjacent to $\gamma M$ the field $f$ coincides with an element of $F(\sigma, \varphi, \gamma M)$. Thus the product $g_{2 m_{\pi}} \circ \cdots \circ g_{1}$ reduces to $g_{i_{2}} \circ g_{i_{1}}$, and this latter is $\varphi(\tau(\pi, \gamma M))^{-1}$. We thus get $h_{\sigma^{\prime}}(\tau(\pi, \gamma M))=h_{\sigma}(\tau(\pi, \gamma M)) \circ \varphi(\tau(\pi, \gamma M))^{-1}=\varphi\left(\tau(\pi, \gamma M)^{\prime}\right)^{-1}$, and then set $\varphi^{\prime}(\tau(\pi, \gamma M))=1$ and $\varphi^{\prime}\left(\tau(\pi, \gamma M)^{\prime}\right)=\varphi\left(\tau(\pi, \gamma M)^{\prime}\right)$.

If we extend $\varphi^{\prime}$ outside $\mathcal{T}$ by setting $\varphi^{\prime}=1$ we obtain a $\Gamma^{\prime}$-invariant rank-2 field that decomposes the holonomy of $\sigma^{\prime}$ and has the required properties. 


\subsection{Finding a virtually invariant system of local reflections without holo- nomy}

Theorem 14.17 Assume $(W, S)$ is two-dimensional and $I$ does not contain any subset $\{i, j, \ell\}$ with $m_{i j} \leq 3, m_{j \ell}=3, m_{\ell i}<+\infty$ (so that $X$ satisfies condition $\left(\mathrm{C}^{4}\right)$ by Lemma 11.4).

Let $\Gamma$ denote a residually finite uniform lattice of $\operatorname{Aut}_{\mathrm{rk}}(A)$ in which the finite index subgroups of $e-w a l l$ stabilizers are separable. Then $\Gamma$ has a finite index subgroup preserving a system of local reflections without holonomy.

Proof First by Corollary 14.9, there is a finite index subgroup of $\Gamma$ acting as a clean uniform lattice. So we may and will assume that $\Gamma$ is clean. By Lemma 14.1, the group $\Gamma$ preserves a system of local reflections $\sigma$. By Remark 14.14 the system $\sigma$ admits a $\Gamma$-invariant decomposition $\varphi$.

We choose $e$-walls $M_{1}, \ldots, M_{n}$ such that any $e$-wall of $A$ is a $\Gamma$-translate of a unique $e$-wall of the family $\left\{M_{1}, \ldots, M_{n}\right\}$.

We first set $\Gamma^{0}=\Gamma, \varphi^{0}=\varphi$ and $\sigma^{0}=\sigma$. We apply Theorem 14.15 to the $e$-wall $M_{1}$, using Remark 10.3. Thus we find a finite index normal subgroup $\Gamma^{1} \subset \Gamma$, a system of local reflections $\sigma^{1}$ and a rank-2 field $\varphi^{1}$ such that $\sigma^{1}$ and $\varphi^{1}$ are $\Gamma^{1}$-invariant, $\varphi^{1}$ is a decomposition of the holonomy of $\sigma^{1}$ and for every polygon $\pi$ either $\pi$ is separated by no $\Gamma$-translate and $\varphi^{1}=\varphi^{0}$ on $\mathcal{T}_{\pi}$ or $\pi$ is separated by some $\Gamma$-translate $\gamma M$ and then $\varphi^{1}(\tau(\pi, M))=1, \varphi^{1}\left(\tau(\pi, M)^{\prime}\right)=\varphi^{0}\left(\tau(\pi, M)^{\prime}\right)$.

We apply again Theorem 14.15 to the subgroup $\Gamma^{1}$, the system of local reflections $\sigma^{1}$, its decomposition $\varphi^{1}$ and the $e$-wall $M_{2}$, thus getting a finite index normal subgroup $\Gamma^{2} \subset \Gamma$ with $\Gamma^{2} \subset \Gamma^{1}$, together with a system $\sigma^{2}$ and a decomposition $\varphi^{2}$ of the holonomy of $\sigma^{2}$ which is trivial along $\Gamma$-translates of $M_{2}$. If we repeat this procedure until we reach the $\Gamma$-translates of $M_{n}$, we obtain sequences $\Gamma^{1} \supset \cdots \supset \Gamma^{n}$ (each normal of finite index in $\Gamma$ ), $\sigma^{1}, \ldots, \sigma^{n}$ systems of local reflections, $\varphi^{1}, \ldots, \varphi^{n}$ with $\sigma^{i}, \varphi^{i}$ invariant under $\Gamma^{i}, \varphi^{i}$ decomposition of the holonomy of $\sigma^{i}$.

We claim that $\varphi^{n}=1$, so that $\sigma^{n}$ has no holonomy.

Indeed consider a polygon $\pi$ with $2 m$ edges. The $e$-walls separating $\pi$ are in the $\Gamma$-orbit of $M_{i_{1}}, \ldots, M_{i_{2 m}}$ with $i_{1}<\cdots<i_{2 m}$. We denote by $N_{j}$ the $\Gamma$-translate of $M_{i_{j}}$ which separates $\pi$.

For $i \notin\left\{i_{1}, \ldots, i_{2 m}\right\}$ we know that $\varphi^{i}=\varphi^{i-1}$ on $\mathcal{T}_{\pi}$.

To simplify notation set $\tau=\tau\left(\pi, N_{1}\right)$. Then we know that $\varphi^{i_{1}}\left(\tau^{\prime}\right)=\varphi^{i_{1}-1}\left(\tau^{\prime}\right)$ and $\varphi^{i_{1}}(\tau)=1$. Half of the $e$-walls $N$ that separate $\pi$ satisfy $\tau(\pi, N)=\tau$, and the 
other half satisfy $\tau(\pi, N)=\tau^{\prime}$. So there is a least positive integer $\ell(\leq m+1)$ such that $\tau\left(\pi, N_{\ell}\right)=\tau^{\prime}$. For every integer $1 \leq j<\ell$ we have $\tau\left(\pi, N_{j}\right)=\tau$ and in fact $\varphi^{i_{j}}=\varphi^{i_{1}}$ on $\mathcal{T}_{\pi}$.

The new decomposition $\varphi^{i_{\ell}}$ is trivial on $\tau^{\prime}$, and takes the same value as $\varphi^{i_{1}}$ on $\tau$. This means that $\varphi^{\ell}=1$ on $\mathcal{T}_{\pi}$.

For $j>\ell$ the decompositions $\varphi^{j}$ keep the value of the previous $\varphi^{j-1}$ on one (or both) of the triangles of $\mathcal{T}_{\pi}$ and puts the value 1 on the other. So $\varphi^{n}=\varphi^{\ell}=1$ on $\mathcal{T}_{\pi}$.

Definition 14.18 Let $X$ denote some polygonal complex. Assume $X$ is equipped with a piecewise Euclidean nonpositively curved length metric $d$. A subgroup $\Lambda$ of $\operatorname{Aut}(X)$ is $d$-convex whenever there is a convex subcomplex $C \subset X^{\prime}$ of the first barycentric subdivision such that $C$ is invariant under $\Lambda$ and $\Lambda$ is discrete cocompact on $C$.

For example if $X$ is the polygonal complex of a two-dimensional Coxeter system $(W, S)$, and if $X$ is endowed with the $\left(\mathrm{C}^{4}\right)-$ or the $\left(\mathrm{C}^{2}\right)$-metric, we will consider $\left(\mathrm{C}^{4}\right)$-convex subgroups or $\left(\mathrm{C}^{2}\right)$-convex subgroups.

Example 14.19 Assume that a polygonal complex $X$ satisfies $\left(\mathrm{C}^{4}\right)$. Then by Lemma 12.6 , any geometric $e$-wall is a convex subcomplex of $A$, endowed with the $\left(\mathrm{C}^{4}\right)-$ metric. The stabilizer of an $e$-wall $M$ in a uniform lattice $\Gamma$ of $X$ has finitely many orbits in $M$. Indeed there are finitely many orbits of oriented edges of $M$ under $\Gamma$, and if an element $\gamma \in \Gamma$ sends $\vec{e} \in M$ inside $M$, then in fact $\gamma M=M$.

It follows that the stabilizer of an $e$-wall is cocompact on the associated geometric $e$-wall, hence is $\left(\mathrm{C}^{4}\right)$-convex, and so is any finite index subgroup of the $e$-wall stabilizer.

Similarly if $X$ satisfies $\left(\mathrm{C}^{2}\right)$ then any finite index subgroup of the stabilizer of a wall in a uniform lattice of $X$ is a $\left(\mathrm{C}^{2}\right)$-convex subgroup.

Note that the trivial subgroup $\{1\}$ is $d$-convex for any metric $d$.

Theorem 14.20 Assume $(W, S)$ is two-dimensional and $I$ does not contain any subset $\{i, j, \ell\}$ with $m_{i j} \leq 3, m_{j \ell}=3, m_{\ell i}<+\infty$, so that $X$ satisfies $\left(\mathrm{C}^{4}\right)$.

Let $\Gamma$ denote a uniform lattice of $\operatorname{Aut}_{\mathrm{rk}}(A)$ in which each $\left(\mathrm{C}^{4}\right)$-convex subgroup is separable. Then $\Gamma$ is commensurable in $\operatorname{Aut}_{\mathrm{rk}}(A)$ with $W$.

Proof The condition implies that $\Gamma$ is residually finite and that the finite index subgroups of $e$-wall stabilizers are separable (see Example 14.19). By Theorem 14.17, 
the group $\Gamma$ has a finite index subgroup $\Gamma^{\prime}$ preserving a system of local reflections $\sigma$ without holonomy. Now by Corollary 13.5, there is an automorphism $\varphi$ of $\operatorname{Aut}_{\mathrm{rk}}(A)$ sending $\sigma$ onto $\sigma^{W}$. Also, conjugation by $\varphi$ identifies $\operatorname{Aut}_{\mathrm{rk}}(A, \sigma)$ with $\operatorname{Aut}_{\mathrm{rk}}\left(A, \sigma^{W}\right)$, an extension of $W$ of finite index. Since $\Gamma^{\prime}$ is a uniform lattice, it has finite index in the uniform lattice $\operatorname{Aut}_{\mathrm{rk}}(A, \sigma)$. Thus conjugation by $\varphi$ maps $\Gamma^{\prime}$ onto a group $\Gamma^{\prime \prime}$ such that $\Gamma^{\prime \prime} \cap W$ is of finite index in both $\Gamma^{\prime \prime}$ and $W$. The commensurability follows.

Proof of Theorem 1.7 The negative curvature condition insures that $(W, S)$ is twodimensional and that $I$ contains no subset $\{i, j, \ell\}$ with $m_{i j} \leq 3, m_{j \ell}=3, m_{\ell i}<+\infty$, so that $X$ satisfies $\left(\mathrm{C}^{4}\right)$ (see Example 11.5).

Clearly $\left(\mathrm{C}^{4}\right)$-convex subgroups are quasiconvex subgroups. So if all quasiconvex subgroups are separable Theorem 14.20 applies.

\section{References}

[1] I Agol, D D Long, A W Reid, The Bianchi groups are separable on geometrically finite subgroups, Ann. of Math. (2) 153 (2001) 599-621 MR1836283

[2] D Angluin, A Gardiner, Finite common coverings of pairs of regular graphs, J. Combin. Theory Ser. B 30 (1981) 184-187 MR615312

[3] H Bass, R Kulkarni, Uniform tree lattices, J. Amer. Math. Soc. 3 (1990) 843-902 MR1065928

[4] A Borel, Compact Clifford-Klein forms of symmetric spaces, Topology 2 (1963) 111122 MR0146301

[5] N Bourbaki, Éléments de mathématique. Fasc. XXXIV. Groupes et algèbres de Lie. Chapitre IV: Groupes de Coxeter et systèmes de Tits. Chapitre V: Groupes engendrés par des réflexions. Chapitre VI: systèmes de racines, Actualités Scientif. et Industr., No. 1337, Hermann, Paris (1968) MR0240238

[6] M Bourdon, Immeubles hyperboliques, dimension conforme et rigidité de Mostow, Geom. Funct. Anal. 7 (1997) 245-268 MR1445387

[7] M Bourdon, Sur les immeubles fuchsiens et leur type de quasi-isométrie, Ergodic Theory Dynam. Systems 20 (2000) 343-364 MR1756974

[8] M R Bridson, A Haefliger, Metric spaces of non-positive curvature, Grundlehren series 319, Springer, Berlin (1999) MR1744486

[9] K S Brown, Cohomology of groups, Graduate Texts in Math. 87, Springer, New York (1982) MR672956

[10] M Burger, S Mozes, Lattices in product of trees, Inst. Hautes Études Sci. Publ. Math. (2000) 151-194 (2001) MR1839489 
[11] M W Davis, Groups generated by reflections and aspherical manifolds not covered by Euclidean space, Ann. of Math. (2) 117 (1983) 293-324 MR690848

[12] M W Davis, Buildings are CAT(0), from: "Geometry and cohomology in group theory (Durham, 1994)”, London Math. Soc. Lecture Note Ser. 252, Cambridge Univ. Press, Cambridge (1998) 108-123 MR1709955

[13] M Davis, T Januszkiewicz, R Scott, Nonpositive curvature of blow-ups, Selecta Math. (N.S.) 4 (1998) 491-547 MR1668119

[14] R Gitik, Doubles of groups and hyperbolic LERF 3-manifolds, Ann. of Math. (2) 150 (1999) 775-806 MR1740992

[15] E Green, Graph products, PhD thesis, Univ. of Warwick (1991)

[16] M Gromov, Hyperbolic groups, from: "Essays in group theory", Math. Sci. Res. Inst. Publ. 8, Springer, New York (1987) 75-263 MR919829

[17] M Gromov, I Piatetski-Shapiro, Nonarithmetic groups in Lobachevsky spaces, Inst. Hautes Études Sci. Publ. Math. (1988) 93-103 MR932135

[18] F Haglund, Les polyèdres de Gromov, C. R. Acad. Sci. Paris Sér. I Math. 313 (1991) 603-606 MR1133493

[19] F Haglund, Réseaux de Coxeter-Davis et commensurateurs, Ann. Inst. Fourier (Grenoble) 48 (1998) 649-666 MR1644077

[20] F Haglund, Existence, unicité et homogénéité de certains immeubles hyperboliques, Math. Z. 242 (2002) 97-148 MR1985452

[21] F Haglund, Complexes simpliciaux hyperboliques de grande dimension, Preprint 200371, Orsay, Orsay (2003)

[22] F Haglund, Finite index subgroups of graph products, to appear in Geom. Dedicata (2006)

[23] F Haglund, F Paulin, Simplicité de groupes d'automorphismes d'espaces à courbure négative, from: "The Epstein birthday schrift", (I Rivin, C Rourke, C Series, editors), Geom. Topol. Monogr. 1 (1998) 181-248 MR1668359

[24] F Haglund, F Paulin, Constructions arborescentes d'immeubles, Math. Ann. 325 (2003) 137-164 MR1957268

[25] F Haglund, D Wise, Coxeter Groups are Special, in preparation

[26] F Haglund, D Wise, Special cube complexes, Submitted (2005)

[27] T Hsu, D T Wise, On linear and residual properties of graph products, Michigan Math. J. 46 (1999) 251-259 MR1704150

[28] T Januszkiewicz, J Świątkowski, Commensurability of graph products, Algebr. Geom. Topol. 1 (2001) 587-603 MR1875609

[29] T Januszkiewicz, J Świątkowski, Simplicial nonpositive curvature, submitted (2005) 
[30] I Kapovich, D T Wise, The equivalence of some residual properties of word-hyperbolic groups, J. Algebra 223 (2000) 562-583 MR1735163

[31] F T Leighton, Finite common coverings of graphs, J. Combin. Theory Ser. B 33 (1982) 231-238 MR693362

[32] G A Margulis, Arithmeticity of the irreducible lattices in the semisimple groups of rank greater than 1, Invent. Math. 76 (1984) 93-120 MR739627

[33] J Meier, When is the graph product of hyperbolic groups hyperbolic?, Geom. Dedicata 61 (1996) 29-41 MR1389635

[34] MS Raghunathan, Torsion in cocompact lattices in coverings of $\operatorname{Spin}(2, n)$, Math. Ann. 266 (1984) 403-419 MR735524

[35] B Rémy, Topological simplicity, commensurator super-rigidity and non-linearities of Kac-Moody groups, Geom. Funct. Anal. 14 (2004) 810-852 MR2084981

[36] M Ronan, Lectures on buildings, Perspectives in Mathematics 7, Academic Press, Boston (1989) MR1005533

[37] C P Rourke, B J Sanderson, Introduction to piecewise-linear topology, Springer, New York (1972) MR0350744

[38] P Scott, Subgroups of surface groups are almost geometric, J. London Math. Soc. (2) 17 (1978) 555-565 MR0494062

[39] B A F Wehrfritz, Generalized free products of linear groups, Proc. London Math. Soc. (3) 27 (1973) 402-424 MR0367080

[40] D T Wise, Non-positively curved squared complexes, aperiodic tilings, and nonresidually finite groups, $\mathrm{PhD}$ thesis, Princeton University (1996)

[41] D T Wise, The residual finiteness of negatively curved polygons of finite groups, Invent. Math. 149 (2002) 579-617 MR1923477

[42] D T Wise, Cubulating small cancellation groups, Geom. Funct. Anal. 14 (2004) 150214 MR2053602

[43] D T Wise, Subgroup separability of the figure 8 knot group, Topology 45 (2006) 421463 MR2218750

Laboratoire de Mathématiques, Université de Paris XI (Paris-Sud)

91405 Orsay, France

frederic.haglund@math.u-psud.fr

Received: 25 July 2005

Algebraic 83 Geometric Topology, Volume 6 (2006) 